\title{
Distribution of Crops and Cropping Patterns in Bangladesh
}

\author{
M Nasim¹, S M Shahidullah ${ }^{1 *}$, A Saha $^{1}$, M A Muttaleb ${ }^{1}$, T L Aditya ${ }^{2}$, M A Ali² and M S Kabir ${ }^{3}$
}

\begin{abstract}
Agricultural land use and distribution of cultivated crops expressed in cropping pattern $(\mathrm{CP})$ constitute the base for increasing produtivity. An in-depth study was conducted on the existing CPs of Bangladesh in each and every upazila (sub-districts) using semi structured questionnaire and data validation by the stakeholder consultation workshop conducted in each of 64 districts. Three hundred and sixteen $\mathrm{CPs}$ were identified throughout Bangladesh excluding the very minor ones. Topmost five CPs were only rice containing $\mathrm{CPs}$, which covered $51 \%$ of the net cropped area. The most dominant $\mathrm{CP}$ was BoroFallow-T. Aman covering 27\% of net cropped area. Results on major cropwise CPs, location-wise CPs, $\mathrm{CP}$ diversity and crop diversity etc are also presented herein.
\end{abstract}

Key words: Land use, cropping pattern, cropping intensity, crop diversity index

\section{INTRODUCTION}

Bangladesh, an agriculture dominating country, belongs to the greatest delta (GangaBrahmaputra and Sundarbans) of the world criss-crossed by thousands of rivers and revulets (Fig. 1). The land is enreached with fertile alluvial soil. The soil and environment is well suited for different kinds of crops all over the year. About $57 \%$ of its total land is arable (Anonymous, 2016a). The lion share of the total land has been brought under cultivation to satisfy the demand of teeming millions. Despite this achivement, a huge population has a limited access to enough land under their jurisdiction making agriculture a challenging option. On the other hand, the yearly transformation of a certain area $(0.47 \%)$ of arable land from agriculture to non-agriculture use is a grave concern to agricultural community due to population pressure, urbanization and some non-agricultural purposes. Thus, getting more food from less land would be one of the most challenging concern for the country even having some improvement of the existing agricultural system. Despite the reduction of dependency on absolute agriculture, $45 \%$ of the total labour forces of the country and 16\% of GDP have to rely directly on agriculture (Anonymous, 2016a). Beside the crop coverage, the agricultural land is occupied by forest, mangrove forest, river, lake, bil, haor, aquaculture, tea and salt pan (Hasan et al., 2013). Lots of crops are cultivated both of tropical and temperate origin in this country. Agricultural land use at a local level is expressed by the spatial and temporal distribution of crops often expressed as cropping pattern (CP). $\mathrm{CP}$ depends on the physiography, environment and socioeconomic conditions of a particular area.

Physiography, ecosystem and environment Agriculture is predominantly influenced by the varying environmental and physiographic conditions of an area. Three broad physiographic groups are marked in Bangladesh and they belong to three distinct geographical ages as, tertiary hills, pleistocene terraces and recent floodplains (Fig. 2). These physiographic groups are represented by hill soils in southeastern and north-eastern areas, accounting for about $12 \%$ of the land, terrace soils consist

${ }^{1}$ Rice Farming Systems Division, BRRI, Gazipur; ${ }^{2}$ Director, Bangladesh Rice Research Institute; ${ }^{3}$ Director General, Bangladesh Rice Research Institute, Gazipur; 'Corresponding author's E-mail: shahidullah4567@gmail.com 


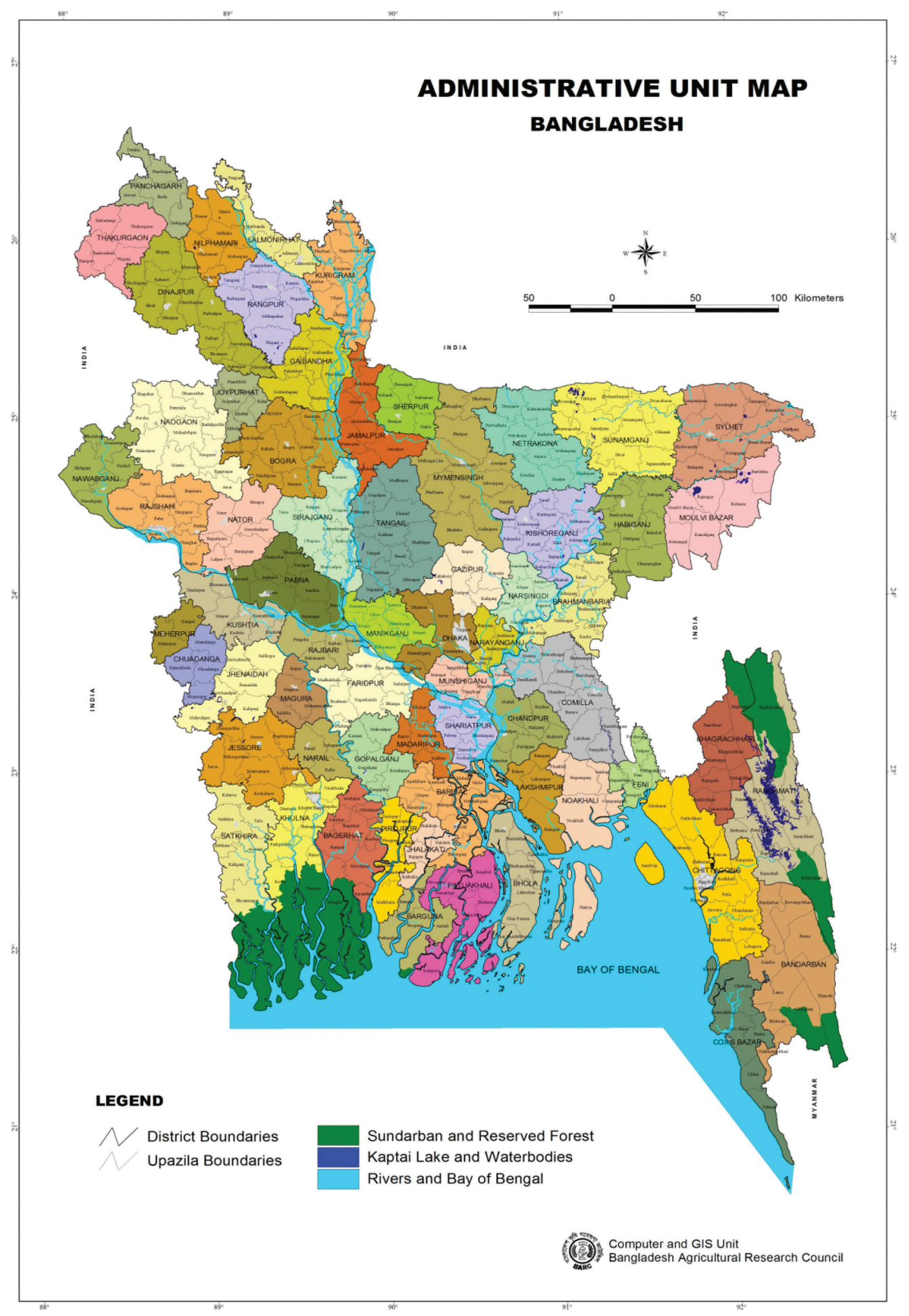

Fig. 1. Upazila-wise map of Bangladesh (http://maps.barcapps.gov.bd/index.php?t=administrative). 


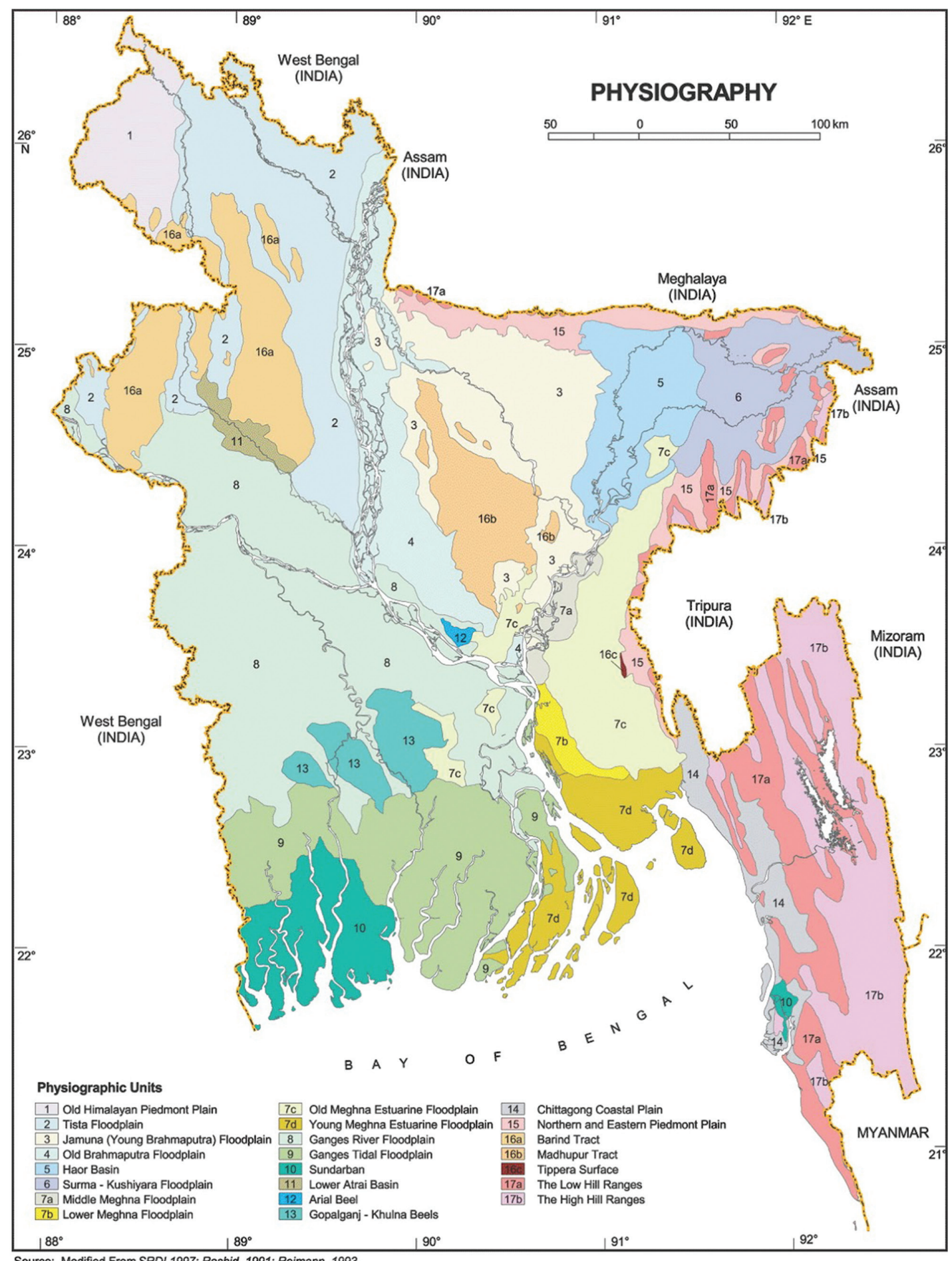

Fig. 2. Soil physiography and general soil type map of Bangladesh (http://en.banglapedia.org/index. php?title=Physiography). 
of two uplifted blocks in the west and centre of the country called Barind and Madhupur Tracts, respectively accounting for about $8 \%$ of the land and floodplain soils distributed across the country accounting for about $80 \%$ of the land. Soil types within these physiographic groups are classified into 21 general types (Fig. 2 ). The cultivation practices and type of crops are exclusively dependent on these soil types (FAO, 1988). In addition to physiography and general soil types, water plays a vital role on crop production. As per FAO (1988), there are five classes of soil based on land level in relation to seasonal flooding. Water is generally scarce for cultivation in high and medium highlands. Contrarily medium lowland, lowland and very lowland are prone to flood of varying degrees during monsoon (Fig. 3). Beside these, there are special types of flood-prone areas mostly confined to the northeastern corner of the country called haor, a saucer-shaped vast depressed area contains bil in it and is inundated by water during monsoon and is dried up in winter (Fig. 4). Tidal wetland, another type of land at the southern part of the country greatly influences the crop production practices of the area. These areas are flood free zones, however are experienced of high and low tide every six hours. The tidal wetland is of two types, saline and non-saline. The saline water obviously restricts crop production severely. There are lot of charlands along the rivers and the coastal belt. Most of the areas having favourable environments for growing crops (Fig. 4). However, a significant area with adverse growing conditions are not unlikely depending growing season when the crop is in progress in the field.

Subtropical monsoon climate prevails in Bangladesh, which is characterized by wide seasonal variation and uneven distribution of temperature and rainfall. Monsoon wind flow plays a vital role in crop production. During warmer season, the wind brings moisture with it to rain in the rainy season. Along with the progress of the rainy season, the major rivers flow with water from the Himalya up to the brim causing flood across the country. In winter, temperature comes down and monsoon wind ceases. As a result, cold wind flows from the north towards the bay. The cold wind mainly comes from the Himalaya, which further lowers the temperature. The winter is designated as the dry parts of the year when temperature might go down as low as $4-5^{\circ} \mathrm{C}$. In contrast, the temperature may goes up beyond $40^{\circ} \mathrm{C}$ in the summer in some places in Bangladesh. The extreme climatic events are not uncommon. The intensity of these events vary with season or geographical location of the country.

In respect to the suitability of growing crops, FAO (1988) classified the whole land into 30 Agroecological regions (AEZ) and 88 subregions (Fig. 5). In this system, information on the environment, which is relevant for land use, was used for the assessment of agricultural potential. By adding and superimposing these information, those regions and subregions were classified. The following information was taken into account: 1. Physiography (Land forms and soil parent material), 2. Depth and duration of seasonal flooding, 3. Length of rainfed Kharif and Rabi growing periods, 4 . Length of preKharif period of unreliable rainfall, 5. Length of the cool winter period and 6. Frequency of occurrence of extremely high $\left(>40^{\circ} \mathrm{C}\right)$ summer temperature.

\section{Agricultural land use}

As described above, the country experiences a lot of environmental variations, so is the case for land use patterns. The land use in croplands involves: Single crop, double crop, triple crop, quadruple crop, current fallow etc The agricultural land use is highly dynamic in Bangladesh.

Among the cultivated crops, rice occupies about $75 \%$ of total cropped areas (Anonymous, 2016a). So all the other crops together, occupy the rest of the cropped area. Rice has the wide adaptation ability under different agroecological niches of Bangladesh. It can be cultivated from the slope of the hill to a very deep flooded areas where water depth rises around $3 \mathrm{~m}$. It can be cultivated thorughout the year with an adjustment of not experience of 


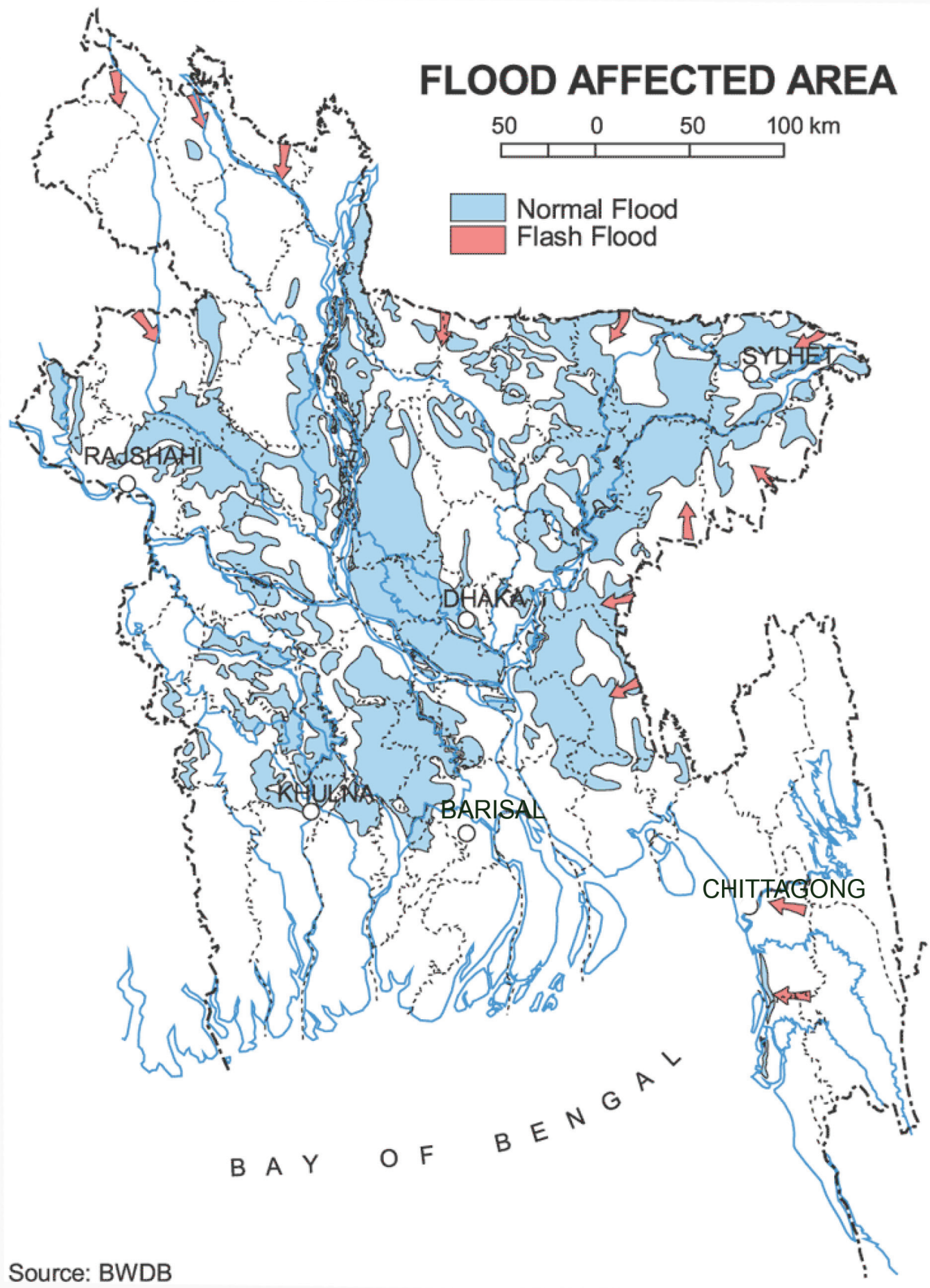

Fig. 3. Flood affected areas of Bangladesh (http://lib.pmo.gov.bd/maps/images/bangladesh/Flood.gif). 


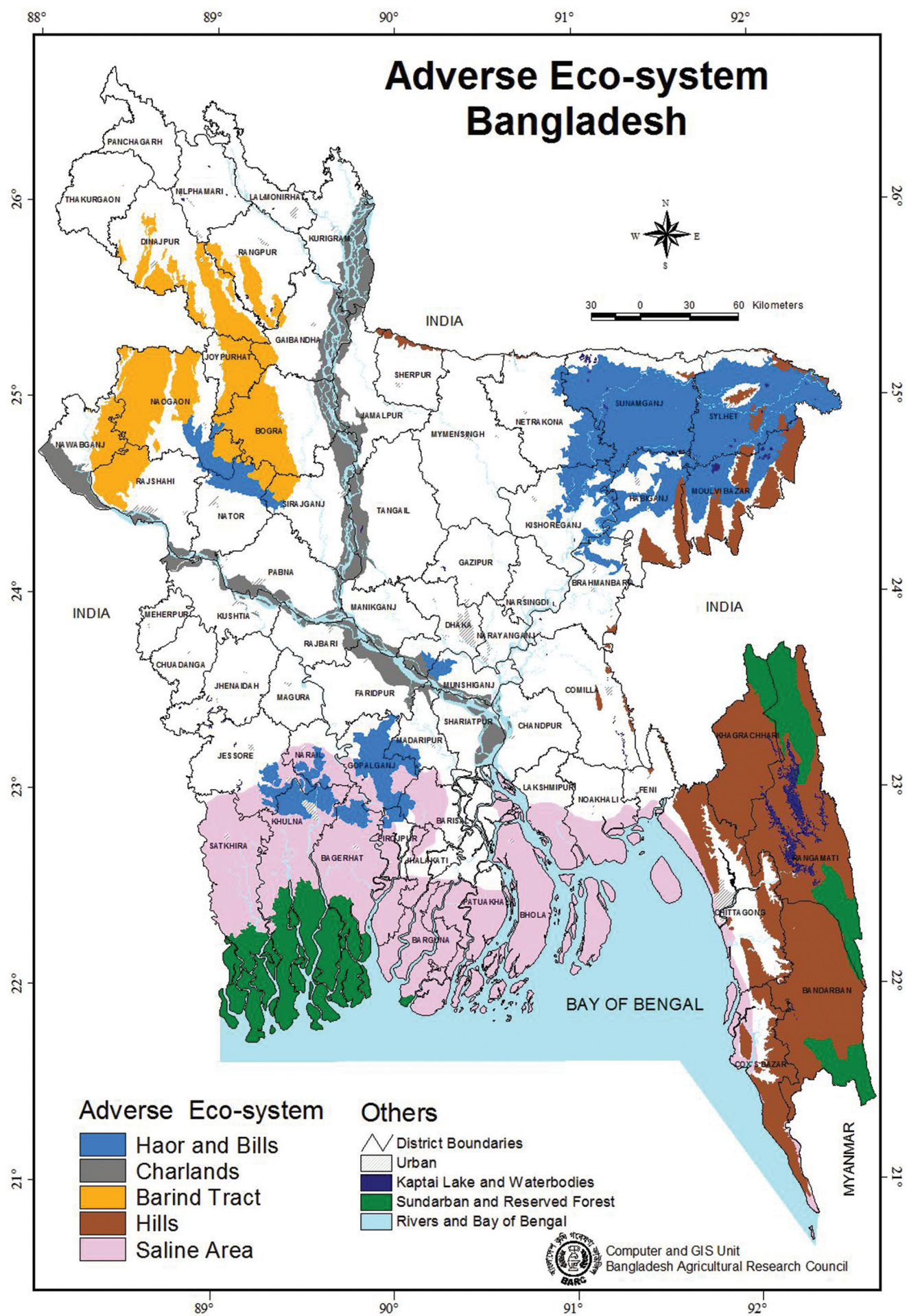

Fig. 4. Adverse ecosystem map of Bangladesh (http:// maps.barcapps.gov.bd/index.php?t=adverse_echo_system). 


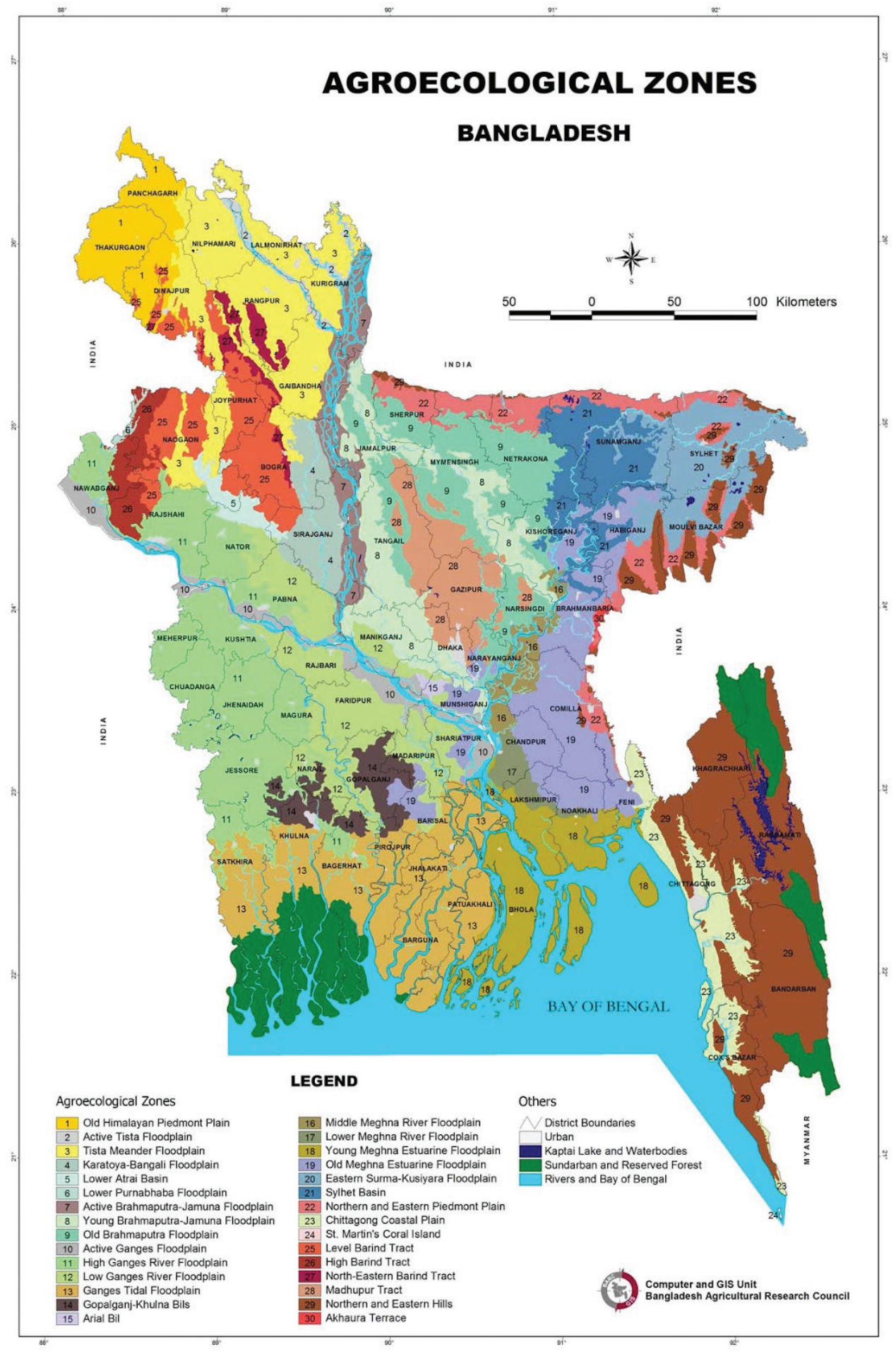

Fig. 5. Agroecological zone map of Bangladesh (http://maps.barcapps.gov.bd/index.php?t=edaphic). 
extreme temperature during the reproductive phase. Rice is the best-adapted cereal crop in the lowland soil in the wet season. No other important crops have this ability to cope with the situation. When the vast areas of our country go under flood water for considerable time in the wet season, or when intermittent flash flood affects majority of our lowlands, or when tide water rises up and falls down twice a day, rice is the only crop option to be suited in those conditions. Thus rice enables us to bring these vast areas under cultivation in unfavourable conditions.

\section{Rice growing environments}

The International Terminology for Rice Growing Environments (Khush, 1984) established a standard classification of rice ecosystems. In this system, primary classes are based on broad areas of sustained water depth. Secondary classes are based on subdivisions of water depths; the dynamics of the water regime, including the dependability of water supply; and on soil constraints in some cases. All classes of rice growing environments are widely present in Bangladesh. The primary classes are briefly defined below:

Irrigated ecosystem. The lands are irrigated with good water control and have adequate water supply throughout the growing season. Rainfall may supplement irrigation water. Rice is transplanted or direct seeded on puddle soil on levelled and bunded field. Lands represent harmonious nature of irrigated areas. Low lying areas, especially flood-prone areas are the most suitable areas. Most of the rice production comes from this ecosystem. In Bangladesh, they are scattered all over the country.

Rainfed lowland. Rainfed lowlands are usually in medium highland areas where rice cultivation depends on rainfall. After onset of rainfall, at some time, rain water accumulates on lands because of continuous and heavy rains. The soil is then puddled and rice is usually transplanted on levelled or slightly slopping bunded or dyked fields with variable depth and duration of flooding of rainfall. Soils alternate from flooded to nonflooded conditions and thus short period of moisture stress or mild submergence may occur. The land is often submersed by flash flood. The water depth varies up to $100 \mathrm{~cm}$ and duration of flooding is usually not more than 10-12 days. Supplementary irrigation may be necessary. Both drought and flooding can occur within the same cropping season.

Deep water. The lowlying lands where flood water accumulates during rainy season and standing water depth vary from $50 \mathrm{~cm}$ to more than $3 \mathrm{~m}$ and flooding occurs only during part of the growing season is called deep water rice (DWR) ecosystem. Here rice is direct seeded on unbunded fields. Soil cycles from flooded to nonflooded conditions. Rice grows 50 to 60 days in rainfed field subject to drought or shallow flooding then is flooded to a depth of more than half a meter for a month or longer. The rice plant grows as flood water rises and rice is harvested after flood water recedes. Rice is often only the crop that can be grown in the flood-prone areas during flooding period. Catling et al. (1988) defined DWR as rice that is usually grown on land that is flooded to more than $50 \mathrm{~cm}$ depth for one month or more during the growing season. DWR at the early stage suffer from drought and with the onset of monsoon, it suffers from variable degrees of flooding that may last up to the reproductive stage. DWR ecosystem is different from tidal wetlands where water may rise more than 50 $\mathrm{cm}$ by tidal action but only for a short period in each day and from shallow flash-flood areas where rice may be submerged $50 \mathrm{~cm}$ or more but for up to 10-12 days.

Upland. Naturally well-drained soil with bunds or unbunded fields without surface water accumulation is called upland. Water does not stagnant even after heavy rains. Lands are level to steeply sloping. Crops suffer from lack of moisture and inadequate nutrition. Rice is direct seeded. Upland rice fields are mostly cultivated using traditional methods (predominantly slash and burn) in scattered fields. Uplands may encounter severe problems 
with hard-pans, low $\mathrm{pH}$ sub soil, rapid onset of drought and lack of soil moisture. In the uplands, soils remain aerobic throughout the season.

Tidal wetland. Tidal wetlands are near the sea coasts and inland estuaries that are directly or indirectly influenced by tides. They are heterogeneous environments. Freshwater tidal wetlands in Bangladesh occur near inland estuaries some distance from the coast. There is little or no intrusion of saline water. The usual feature of these areas is the occurrence of daily tidal flooding between 30 to $60 \mathrm{~cm}$ depth, with some extremes up to $80 \mathrm{~cm}$ from June to September then falling sharply to 20 $\mathrm{cm}$ in late September. The field condition in the high tide is unsuitable for any crop but rice. The water levels in coastal rice fields fluctuate. Transplanted rice is grown in this environment and they suffer from daily high and low tidal pressure and also high depth of standing water. Tall rice seedlings with quick growing nature are transplanted to withstand submergence and tidal pressure as tides rise and fall. Saline water tidal wetland occurs near sea coast and mouth of estuaries and sea water intrusion takes place. The soil may have severe problems of salinity and other toxicity. The soil comes in contact with sea water by tidal flooding, direct inundation of sea water and by saline underground water. However, in the rainy season rain water dilutes salts of the top soil and washes away and leaches the salts and at that time intrusion of saline water is also checked by fresh water in the upstream river. So, rice production is possible at that time. The problem of salinity increases in the dry season.

In Bangladesh rice is grown in all these ecosystems. Our rice seasons generally fit into the primary classes of rice ecosystems. Boro represents irrigated ecosystem, T. Aman represents rainfed lowland and tidal wetland, Aus represents upland and B. Aman represents deep water rice ecosystem. However, with the modern rice varieties, the association between rice ecotypes and ecosystems is fading (Miah et al., 2004).

\section{Non-rice crops growing environment}

Wheat is cultivated in Rabi season and it needs prolonged winter. It is best suited in well drained sandy loam soil, however, it is cultivated in range of sandy loam to clay loam soils. It is also suited to medium highland to medium lowland. Wheat is best suited in cold winter prone areas of northern districts. Wheat covers $5.23 \%$, of the net cropped areas (NCA) (Anonymous, 2016b). Maize is cultivated in two seasons, Rabi and Kharif-I. It is suited best in loamy soils. It is found to be the best in the northern districts. It is also suited in charlands throughout the country. However, in most of the potato growing areas maize is followed after potato in Kharif-I season. Maize covers $3.9 \%$ of the NCA (Anonymous, 2016b).

Many of the varieties of pulses and oil seeds are well suited to our ecosystems. However, pulse and oil seed crops cover only $4.9 \%$ and $5.02 \%$, respectively of the NCA (Anonymous, 2016b). Among the pulses, the major crops are grasspea, lentil, chickpea, blackgram and mungbean and the minor ones are pigeonpea, fieldpea, fava bean and cowpea. Grasspea, lentil, chickpea, fieldpea, cowpea and fava beans are grown during Rabi season. Blackgram is grown just after the peak of monsoon. Mungbean is grown in winter and in the late winter or in Kharif-I seasonas well. Among the oil seed crops, rapeseed and mustard, sesame and groundnut are the major crops while niger, safflower, sunflower and soybean are minor ones. Rapeseed and mustard, niger and linseed are grown in the Rabi season while groundnut, sunflower and soybean in both Rabi and KharifII seasons. Sesame is grown both in Kharif-I and Kharif-II seasons.

Pulses are not evenly distributed in the country. Their cultivation is mainly concentrated within the gangetic floodplain in the northern districts and in some areas of southern districts. Soils of this area are calcareous from top to certain depth, loamy in the ridges and clay in the basin. $\mathrm{pH}$ ranges from 6.5-8. Availability of phosphorus, calcium, molybdenum and boron is relatively high, which are important for grain legumes. Lentil, blackgram and mungbean 
are grown on high and medium high lands, moderately well to poorly drained light textured soils while chickpea and grasspea are grown on medium-high to low lands, poorly drained heavy textured soils. Grasspea is grown almost all over the country in medium low and lowlands. Mungbean is grown in loam soil. It can also perform well in clay soil if it is well drained.

Rapeseed and mustard is best grown in loamy soils. It can also be cultivated in clay loam and silt loam soils. Groundnut is cultivated in sandy loam and sandy soil of charland on the river bank or in the basin. High land with sandy loam soil is suitable for sesame.

Jute is cultivated in loamy soils in medium highland to medium lowland. It requires natural water source near the field for its retting. Jute cultivation is scattered throughout the country, however, is concentrated in greater Faridpur and northern districts. It occupies about 8.05\% of NCA (Anonymous, 2016b).

Sugarcane is cultivated in clay loam, loam and sandy loam soils. It is suited for medium highland and medium lowland. In some sandy loam soils where Boro cultivation is difficult, farmers often choose sugarcane in it. It grows well in the low Ganges river floodplain. Sugarcane covers about $1.25 \%$ of NCA (Anonymous, 2016b).

There are lots of vegetables cultivated in our country. Vegetable is cultivated in fertile and well-drained soil often near the cities and towns. Vegetables are grown round the year in three different seasons. Many temperate season's vegetable are well suited to our winter.

\section{Cropping pattern}

There are many crop growing niches seen in Bangladesh to support an unique biodiversity of crops throughout the year. Types of crops cultivated in an area and round the year is very important to increase the total crop production and productivity in that area. Distribution of crops in an area round the year can be expressed by CP. The yearly sequence and spatial arrangement of crops or crops and seasonal fallow of a given area are known as
CP. CP is an important indicator of land use, environment and socio-economic aspects of farmers of a locality. It indicates the proportion of areas under different crops in a given time. It also indicates the cropping activities in an area. In $\mathrm{CP}$, crops are grown in sequence on same piece of land in a year. Here succeeding crop is established after the preceding crop has been harvested. Thus in CP there is turn around time and may or may not have a fallow period in a season. $\mathrm{CP}$ is very important, it allows increase in intensity and hence total production in a year as well. Climate, environment, resources and socioeconomic conditions determine the $\mathrm{CP}$ in an area. Climate and environments play a vital role. Presence of water is an important factor to choose a crop. Economic consideration of a farmer like irrigation, inputs, value of crops and decision of farmer and communities and government policies are also crucial. Based on appropriate consideration of all these issues farmers make a decision and construct a $\mathrm{CP}$ with judicious sequence.

$\mathrm{CP}$ or crop sequence is practically an annual strategy to optimize agronomic and economic yield in sustainable manner. CP itself is a dominant management practice in agriculture. This management not only help increase total production in a year but it influences many other management practices to be followed in it. It is so vital that many technologies, which are otherwise potential when is practiced individually, fail to show its potentiality when they have to be fitted in CP. CP controls many crop production and soil property issues. Crop diversity, input use, management, weed and disease infestation, soil physical properties like bulk density etc, cover crop, green manure, mulches, organic matter, C-N storage, erosion, water infiltration etc are influenced by $\mathrm{CP}$. Thus CP provides us with a complete picture of agriculture in a locality.

The cropping patterns of Bangladesh are usually rice-based. However, these are diversified as well. The other crops in sequence are selected from the non-rice cerelas, pulses, oil crops, vegetables and fibre crops. Thus ricebased cropping patterns not only provide us 
the major source of dietary energy through rice but also supplements our essential diets.

Before 1970 i.e., before the green revolution era, many cropping patterns were evolved based on the characteristics of traditional varieties, age-old management practices, socioeconomic needs and the existing environments. There has been a lot of changes and re-adjustments since the green revolution era to date. Many new crop varieties and technologies adaptable to the changing environments are coming up. The input-intensive technologies turned many direct seeded rice areas into transplanted rice areas for better efficacy of the inputs and ease of management practices. Irrigation and other technologies turned many Rabi crop growing areas into Boro growing areas. Wheat, maize and potato areas also increased. As a result, many Rabi crops including pulses and oil seeds failed to accommodate in the systems and their areas decreased significantly. Boro areas increased dramatically. DWR and direct seeded Aus and Aman areas with local varieties decreased considerably.

\section{Uniqueness, usefulness and policy relevance of the study in Bangladesh}

Distribution of crops i.e., agricultural land occupied by different crops is an important consideration which is usually expressed by $\mathrm{CP}$. Study of CP has lots of merits. However, only some sporadic studies are found on this subject (Ali, 2014; Chowdhury et al., 2008; Hossain et al., 2016; Shahidullah et al., 2006), they are mostly confined for a small locality. Rice Farming Systems Division of BRRI conducted an earlier study during the end of 1990, which was a bit similar to this study based on data collected from DAE on CP (NurE-Elahi et al., 2001). The study was not very huge, narrow in presentation, however, district wise major CPs were identified. Contrarily, current study is huge. It presents information on $\mathrm{CPs}$ throughout the country in each and every upazila. List of CPs, their area coverage, their relation with other land use parameters, groups of CPs according to major crops and related information is accumulated in this study. Stakeholders need information on crops, their spatial and temporal distribution, their environment and management in different areas of the country, which is very vital in decision making. No study can provide us with this information. This study is a means to solve the gap on this vital aspect of information. This study has many implications to the concerned stakeholders. Farmers will have ideas about his ecosystems and land use and avenue of improvement/intervention. Researchers will have avenues on crops and varieties and their areas of improvement, use of inputs tagged with existing environment and integration of component technologies to get potential yield of the CP. Policy makers and extension personnel will get ideas on material and capital input mobilization, technology transfer etc to explore the potential yield of the system. Thus, this study may be considered as vital and unique by its nature.

\section{Justification and objective of the study}

$\mathrm{CP}$ brings appropriate crops and management in appropriate spaceand time. Improved formation of $\mathrm{CP}$ helps to approach the sustainable food security. The information related to $\mathrm{CP}$ is quite vital to the farmer, researcher and extension experts. Even the policy makers would have the idea to formulate the policy issues from the major CPs of the country. Unfortunately, we have a little updated knowledge about the $\mathrm{CP}$ of the whole country. It is not known how many $\mathrm{CPs}$ are there or what is the most dominant $\mathrm{CP}$ with its area coverage or related information all over the country. It is also not known how many CPs are there with a specific crop in it or $\mathrm{CP}$ without rice crops. The present study is an attempt to answer those questions, and aims to study agricultural land use and $\mathrm{CP}$ and create data base on it across the whole country with a view to exploring the potential of CPs in future from these information. 


\section{METHODOLOGY}

The study was conducted following two steps.

- Collection and analysis of secondary data and find out the mismatching of the data.

- Conducting stakeholder consultation workshop to review, verify and validate mismatch data discussing with the relevant sources and finalize data.

\section{Collection and analysis of secondary data and finding mismatch}

Present study was carried out basically using secondary source of information from the Department of Agricultural Extension (DAE). DAE keeps records of crops in every nook and corner of the country. At the grass root level, in the blocks, Sub Assistant Agriculture Officer (SAAO) collects data from the field. They use Mauza map to identify NCA and the land use for non-agricultural purposes. In the cropped area, major crop growing field and their coverage is also identified. SAAO conducts survey following the possible ways to collect data. They collect the list of farmers and their lands from the manager of each Boro irrigation scheme. They also make list of farmers for the other major crops like T. Aman, Aus, wheat, jute etc and for minor crops as well. They collected data by interviewing farmers who cultivate different crops, use different varieties in respective hecterage. He also uses his own judgment by his own eye estimation. Finally observing the actual harvest area, judging by his own experience, the individual crop, their varieties and coverage are identified. By these ways they keep data of each and every crop and their coverage in his blocks. Combining block data, union, then upazila, then district data are compiled and kept in the record book of DAE. Thus in each upazila, season wise cultivated crops and their coverage are recorded.

DAE also keeps information on CP. Each crop is cultivated in a specific field in a single, double, triple or quadruple CP. Based on individual crops coverage, the area of a crop in a $\mathrm{CP}$ is distributed. Other crops of these $\mathrm{CP}$ have to match in their respective coverage. Thus individual CP coverage data were developed.

Data we used in this study were collected from DAE. A semi-structured questionnaire was developed for data collectionon crop, CP, their area coverage etc for the year of 2014. A small team of investigators visited the Deputy Director (DD), DAE office. They collected the secondary information of crops, their hecterage from each upazila from the district office. The questionnaires were then distributed to each upazila to collect information on CPs and their hectarage and other related issues. Upazila Agriculture Office filled up the questionnaires and sent back to the investigators. These data were analyzed to find out the mismatch of data, if any, among the data and any query regarding them.

\section{Conducting stakeholder consultation workshop}

Two sets of data, one collected from DD Office on crop and their coverage in each upazila and the other collected through questionnaire on $\mathrm{CP}$ and their coverage from Upazila Agriculture Office were analyzed. After analysis of the data, usually there were some mismatches of data and were some information that needed further clarification. To purify and finalize this information stakeholder consultation workshops were conducted in 64 districts separately to work on upazila level data. A team of investigators (researchers) visited each district and organized a workshop. In the workshop, DD and district level all concerned officers of DAE and from upazila level UAO, AEO, UAA, SAPPO, SAAOs of respective upazila attended. In the workshop if there is a mismatch of data or any other query on data, researchers pointed out it for discussion. Then the data were finalized. The whole study was conducted from August 2015 to November 2016, throughout the country.

Thus the data used for final analysis were the overviewed data. The CP for the present study with its hectarage means the proportion of areas under different CPs in each upazila in 2014. 


\section{Analysis of data}

Collected data were analyzed using Micro Soft Excel programme. Tally, addition, average and descriptive statistics were used for presentation of data. Based on these data, tables were constructed and discussed accordingly.

\section{Limitation of the data}

The data were collected from administrative area i.e., from each upazila of the country. So results were presented easily for upazila, district or region. However, among the upazilas there is transboundary ecosystem coverage where parts of the upazila are taken into account. Examples are Barind tract, haor areas, tidal wetlands etc These areas extend transboundary of more than one upazilas. Exact ecosystem-wise result presentation is difficult in this study. Primary level data collection, ground truthing etc were not included in this study.

\section{Relevant terminology}

Land use. The utilization of surface of a land on a specific space at a given time is called land use. When a land is utilized for agricultural purpose, it may be termed as agricultural land use.

$\mathbf{C P}$. The sequence of crops or crops and fallow on a given area in a year. It includes the seasonal and annual crops, not the perennial crops. Seasonal crops can be sequenced by other seasonal crops or fallow. Annual crops may be replaced after its harvest. So, these are included in CP. Perennial crops, orchard crops, tree crops are not sequenced. So they are excluded from the CP. In case of mixed crops or inter crops, the area in which they are cultivated from it, the portion of area of a companion crop occupied is calculated using land equivalent ratio. CP is generally denoted by writing the crop's name one after another separated by dash sign. Serial of crops indicates the sequence, which is demarcated by dash sign. If in one season the land remained fallow, instead of crop name fallow was written. Writing $\mathrm{CP}$ generally started from Rabi or Boro season followed by Kharif-I or Aus then Kharif-II or Aman season. The examples are: Potato-Maize-T. Aman, BoroFallow-Fallow etc. If two crops are grown in one season in a CP, e.g., two potato crops in Rabi season, a dash is used in between the crops e.g., Potato-Potato-Aus- T. Aman. Other than arable crops, e.g., orchard fruit crops, plantation tree, social forest, bamboo bushes, homestead crops, rooftop crops were not considered as crops of $\mathrm{CP}$. Very minor CPs in terms of area coverage, was not included in our CP list. However, they were included in 'Other' category (Table 2) and were used in calculation of $\mathrm{CP}$ of respective upazila.

Fallow. In a temporal distribution of crops in a land in a year, if a season remains uncultivated it is considered as fallow. Fallow lands may be converted to cultivated land. Unfavourable weather like flood or socioeconomic conditions may cause a land to remain fallow.

Current fallow (CF). If a cultivable land remains fallow throughout the year, it is called CF. However, if a land remains fallow in one season, but cultivated in another season in a year, it is not CF. CF was not considered in NCA.

Net cropped area (NCA): A cultivable land may be either cultivated or remained fallow throughout the year. When a land is cultivated, one or two or three crops round the year may be cultivated in it. The summation of cultivated land area of a region is called NCA. Thus, it is the actual area under crop in a specific year. CF is not considered in it.

Single cropped area/DCA/TCA/QCA: If only one crop is cultivated in a year in an arable land, the area is called SCA. Using similar sense double, triple and quadruple crop areas are defined.

Total cropped area. It is the summation of area cultivated once as well as more than once in a particular year. When two crops in sequence are cultivated in a land in a year, the area is counted twice and so on for three and four crops. Thus it is the summation of SCA $x 1$, DCA $\times 2$, TCA $\times 3$ and QCA $\times 4$.

Cropping intensity (CI). CI is the ratio of total cropped area and NCA in a particular region in a year. It practically indicates number of crops cultivated in ayear in a specific land. It is expressed in percentage. 
Annual crops (AC). Annual crops are the crops which are cultivated throughout the year, e.g., sugarcane, banana, papaya. These crops are considered in crops of $\mathrm{CP}$, as in the next year cultivation of these crops may be replaced by other seasonal crops in sequence. As such they are included in NCA. Contrarily in BoroFallow-Fallow CP, Boro crop is not an annual crop but a seasonal crop.

Fruit crops. Permanent perennial fruitbearing crops. They are not considered in $\mathrm{CP}$ as they have no sequence. However, annual fruit crops i.e., banana, papaya etc are differed from it and were considered in crops of $\mathrm{CP}$ as stated above. If arable field crop is cultivated with fruit trees as intercrop, land equivalent ration of the field crop was considered and this field crop was put into CP.

Timber crops and bamboo bushes. Timber producing permanent and perennial trees and bamboo bushes were not considered in $\mathrm{CP}$ as they have no temporal sequence.

Crop diversity index was calculated by using the following equation described by Kshirsagar et al. (1997).

$$
C D I_{i}=1-\sum_{j=o}^{n}\left(\frac{a_{i j}}{A_{i}}\right)^{2}
$$

Where, CDI: Crop Diversity Index

$a_{i j}$ : Area planted to the jth crop in the ith location

$\mathrm{A}_{\mathrm{i}}$ : Total area planted under all crops

The index is zero for a land area growing only one crop. It approaches unity as the level of diversity increases.

\section{RESULTS AND DISCUSSIN}

Many striking results were revealed when data were analyzed by spread-sheet. Table 1 shows the district-wise common land use pattern of Bangladesh as represented by annual crop area, SCA, DCA, TCA, QCA, others, NCA and CI. DCA dominated in different districts in comparison to SCA or TCA except in Narayanganj, Pirojpur, Sirajganj, Sunamganj, Tangail and Rangamati. These districts have more low-lying areas except Rangamati. Therefore, single Boro CP dominated in those districts. Whereas, in Rangamati, single season jhum cultivation is dominated. QCA was extremely low in most of the districts. It was the highest in Bogra followed by Naogoan and Jessore. As QCA was very low, it contributed less to CI i.e., number of crops in yearly sequence. However, the region which had more TCA, consequently had greater CI. TCA was the highest in Bogra district followed by Naogoan and Dinajpur districts. Bogra district had the highest CI followed by Kushtia, Laxmipur and Thakurgoan districts. Lowest CI was found in Sunamganj followed by Rangamati and Gopalganj. Sunamganj and Gopalganj are haor and low lying areas where scope of cultivation of crops is only possible in Rabi season and in Rangamati, a hilly region, only jhum crops are grown in one season. Annual crop (AC) area was the highest in Tangail followed by Natore and Rangamati. Thus these districts produced more banana, papaya, turmeric, ginger etc. Table 24 presents the information on ACs. NCA of the country was more than 8 million hectares. Population pressure, urbanization and industrialization will reduce this figure gradually. However, we have to get our future food from this land. Kabir et al. (2015) mentioned the current NCA to be 7.81 million hectares and they projected it to be $7.71,7.49$ and 7.18 million hectares in 2021, 2031 and 2041, respectively. Mymensingh had the highest NCA followed by Dinajpur and Naogoan districts. Increased NCA might be because of bigger geographical area of the districts and increased arable areas as well. The country's average indicated that DCA was more than the sum of AC, SCA, TCA QCA and more than twice of the SCA or TCA, separately. QCA was quite negligible, which was $0.22 \%$ of the NCA. The average CI was $200 \%$. 
Table 1. District-wise land use in Bangladesh (area in hectare), 2014-15.

\begin{tabular}{|c|c|c|c|c|c|c|c|c|c|}
\hline & District & $\begin{array}{c}\text { Annual } \\
\text { crop }\end{array}$ & SCA & DCA & TCA & QCA & Others & NCA & $\mathrm{CI}(\%)$ \\
\hline 01 & Brahmanbaria & 290 & 59760 & 67390 & 10920 & 50 & 1040 & 139450 & 165 \\
\hline 02 & Bagerhat & 3430 & 48570 & 51750 & 8640 & 0 & 1140 & 113530 & 161 \\
\hline 03 & Bandarban & 13680 & 12110 & 13370 & 930 & 0 & 640 & 40730 & 138 \\
\hline 04 & Barguna & 630 & 19260 & 41180 & 37770 & 0 & 830 & 99670 & 218 \\
\hline 05 & Barisal & 5095 & 33320 & 92355 & 26175 & 0 & 1515 & 158460 & 192 \\
\hline 06 & Bhola & 5205 & 9130 & 105000 & 65995 & 0 & 1050 & 186380 & 228 \\
\hline 07 & Bogra & 1520 & 8940 & 98550 & 106610 & 5070 & 1460 & 222150 & 248 \\
\hline 08 & Chandpur & 945 & 24100 & 50200 & 15480 & 150 & 1105 & 91980 & 190 \\
\hline 09 & Chapainawabganj & 11160 & 15280 & 46170 & 46980 & 0 & 770 & 120360 & 217 \\
\hline 10 & Chittagong & 5180 & 70220 & 88230 & 34785 & 0 & 1715 & 200130 & 180 \\
\hline 11 & Chuadanga & 3400 & 2220 & 55880 & 26110 & 350 & 490 & 88450 & 224 \\
\hline 12 & Comilla & 980 & 45675 & 92540 & 63550 & 2300 & 1855 & 206900 & 210 \\
\hline 13 & CoxBazar & 3830 & 13250 & 52680 & 14475 & 50 & 1095 & 85380 & 197 \\
\hline 14 & Dhaka & 740 & 17970 & 32990 & 15730 & 0 & 710 & 68140 & 196 \\
\hline 15 & Dinajpur & 4145 & 800 & 188505 & 81350 & 210 & 1830 & 276840 & 228 \\
\hline 16 & Faridpur & 5590 & 8530 & 75990 & 48750 & 0 & 1130 & 139990 & 225 \\
\hline 17 & Feni & 280 & 16300 & 49785 & 4810 & 0 & 755 & 71930 & 183 \\
\hline 18 & Gaibandha & 4110 & 10540 & 105980 & 29680 & 0 & 1050 & 151360 & 210 \\
\hline 19 & Gazipur & 7520 & 33775 & 35095 & 8250 & 0 & 730 & 85370 & 161 \\
\hline 20 & Gopalganj & 1790 & 68200 & 32775 & 9665 & 0 & 710 & 113140 & 146 \\
\hline 21 & Habiganj & 1200 & 71350 & 73160 & 21615 & 0 & 1125 & 168450 & 170 \\
\hline 22 & Jamalpur & 2810 & 7950 & 106340 & 42600 & 90 & 1020 & 160810 & 220 \\
\hline 23 & Jessore & 4020 & 19440 & 97740 & 64660 & 2880 & 1230 & 189970 & 225 \\
\hline 24 & Jhalakati & 2830 & 10640 & 29950 & 7870 & 0 & 610 & 51900 & 189 \\
\hline 25 & Jhenaidaha & 10150 & 6790 & 73590 & 47780 & 700 & 840 & 139850 & 223 \\
\hline 26 & Joypurhat & 1500 & 110 & 22425 & 53715 & 0 & 750 & 78500 & 267 \\
\hline 27 & Khagrachhari & 10220 & 13140 & 17960 & 2170 & 0 & 830 & 44320 & 151 \\
\hline 28 & Khulna & 1080 & 54915 & 65495 & 4605 & 0 & 985 & 127080 & 159 \\
\hline 29 & Kishoreganj & 1490 & 111950 & 58035 & 26335 & 350 & 1680 & 199840 & 156 \\
\hline 30 & Kurigram & 1760 & 14030 & 95755 & 36465 & 10 & 1340 & 149360 & 214 \\
\hline 31 & Kushtia & 7280 & 3260 & 48260 & 54410 & 700 & 870 & 114780 & 240 \\
\hline 32 & Lakshmipur & 400 & 6400 & 46210 & 45435 & 450 & 1005 & 99900 & 240 \\
\hline 33 & Lalmonirhat & 1290 & 3090 & 66740 & 26955 & 0 & 765 & 98840 & 223 \\
\hline 34 & Madaripur & 1040 & 16100 & 44890 & 20770 & 0 & 590 & 83390 & 204 \\
\hline 35 & Magura & 1660 & 2580 & 28190 & 42730 & 0 & 650 & 75810 & 251 \\
\hline 36 & Manikganj & 2350 & 11500 & 54070 & 24680 & 0 & 790 & 93390 & 212 \\
\hline 37 & Maulvibazar & 1820 & 48925 & 66270 & 9635 & 0 & 820 & 127470 & 168 \\
\hline 38 & Meherpur & 2340 & 640 & 26900 & 23830 & 0 & 380 & 54090 & 239 \\
\hline 39 & Munsiganj & 190 & 17670 & 39280 & 3870 & 0 & 790 & 61800 & 177 \\
\hline 40 & Mymensingh & 6060 & 34730 & 208220 & 38835 & 100 & 1715 & 289660 & 199 \\
\hline 41 & Naogaon & 2500 & 33690 & 143520 & 86050 & 3530 & 1610 & 270900 & 221 \\
\hline 42 & Narail & 1670 & 6450 & 50330 & 15110 & 0 & 410 & 73970 & 210 \\
\hline 43 & Narayanganj & 180 & 18780 & 17900 & 2410 & 0 & 410 & 39680 & 158 \\
\hline 44 & Narsingdi & 2850 & 20600 & 38220 & 9840 & 0 & 730 & 72240 & 181 \\
\hline 45 & Natore & 17240 & 13150 & 59780 & 44035 & 0 & 1035 & 135240 & 210 \\
\hline
\end{tabular}


Table 1. Continued.

\begin{tabular}{|c|c|c|c|c|c|c|c|c|c|}
\hline & District & $\begin{array}{c}\text { Annual } \\
\text { crop }\end{array}$ & SCA & DCA & TCA & QCA & Other & NCA & CI $(\%)$ \\
\hline 46 & Netrokona & 310 & 85560 & 106545 & 6005 & 0 & 1290 & 199710 & 160 \\
\hline 47 & Nilphamari & 2310 & 2650 & 81340 & 34510 & 0 & 800 & 121610 & 224 \\
\hline 48 & Noakhali & 1540 & 76910 & 84750 & 34215 & 0 & 1115 & 198530 & 178 \\
\hline 49 & Pabna & 5920 & 14020 & 88870 & 74060 & 30 & 1370 & 184270 & 230 \\
\hline 50 & Panchagarh & 2310 & 4130 & 72200 & 22860 & 0 & 710 & 102210 & 216 \\
\hline 52 & Pirojpur & 2130 & 36105 & 35925 & 7255 & 0 & 865 & 82280 & 162 \\
\hline 53 & Rajbari & 2360 & 6670 & 31800 & 34390 & 0 & 740 & 75960 & 234 \\
\hline 54 & Rajshahi & 10420 & 12320 & 86100 & 55820 & 1100 & 1360 & 167120 & 221 \\
\hline 55 & Rangamati & 14380 & 17625 & 10010 & 910 & 0 & 755 & 43680 & 128 \\
\hline 56 & Rangpur & 4350 & 8970 & 101490 & 59140 & 200 & 1100 & 175250 & 227 \\
\hline 60 & Sirajganj & 2440 & 27585 & 111615 & 40645 & 0 & 1235 & 183520 & 206 \\
\hline 61 & Sunamganj & 510 & 206805 & 55400 & 3560 & 0 & 1135 & 267410 & 123 \\
\hline 62 & Sylhet & 1100 & 110350 & 88120 & 8955 & 0 & 1625 & 210150 & 151 \\
\hline 63 & Tangail & 20250 & 18640 & 144385 & 47820 & 250 & 1705 & 233050 & 204 \\
\hline \multirow[t]{2}{*}{64} & Thakurgaon & 2790 & 1710 & 80360 & 62890 & 0 & 640 & 148390 & 240 \\
\hline & Bangladesh & 243765 & 1787595 & 4455305 & 1996365 & 18680 & 64790 & 8566500 & 200 \\
\hline
\end{tabular}

Table 2. List of cropping patterns in Bangladesh, 2014-15.

\begin{tabular}{|c|c|c|c|c|c|}
\hline & Cropping pattern & Area (ha) & $\%$ of NCA & No. of district & No. of upazila \\
\hline 001 & Boro-Fallow-T. Aman & 2306005 & 26.919 & 63 & 426 \\
\hline 002 & Boro-Fallow-Fallow & 1139530 & 13.302 & 59 & 342 \\
\hline 003 & Fallow-Fallow-T. Aman & 509480 & 5.947 & 36 & 162 \\
\hline 004 & Boro-Aus-T. Aman & 209015 & 2.440 & 47 & 177 \\
\hline 005 & Fallow-Aus-T. Aman & 193275 & 2.256 & 30 & 108 \\
\hline 006 & Mustard-Boro-T. Aman & 184620 & 2.155 & 51 & 203 \\
\hline 007 & Boro-B.Aman & 183070 & 2.137 & 32 & 113 \\
\hline 008 & Potato-Boro-T. Aman & 180380 & 2.106 & 33 & 115 \\
\hline 009 & Wheat-Jute-T. Aman & 147210 & 1.718 & 43 & 216 \\
\hline 010 & Vegetable-Vegetable-Vegetable & 143270 & 1.672 & 61 & 283 \\
\hline 011 & Mustard-Boro-Fallow & 143130 & 1.671 & 37 & 112 \\
\hline 012 & Grasspea-Fallow-T. Aman & 108150 & 1.262 & 25 & 80 \\
\hline 013 & Maize-Fallow-T. Aman & 101460 & 1.184 & 39 & 126 \\
\hline 014 & Wheat-Fallow-T. Aman & 90910 & 1.061 & 39 & 100 \\
\hline 015 & Mungbean-Fallow-T. Aman & 89650 & 1.047 & 22 & 70 \\
\hline 016 & Grasspea-Aus-T. Aman & 81610 & 0.953 & 19 & 61 \\
\hline 017 & Vegetable-Fallow-T. Aman & 74710 & 0.872 & 45 & 170 \\
\hline 018 & Vegetable-Vegetable-Fallow & 63935 & 0.746 & 59 & 168 \\
\hline 019 & Onion-Jute-T. Aman & 54185 & 0.633 & 39 & 102 \\
\hline 020 & Mungbean-Aus-T. Aman & 53730 & 0.627 & 14 & 43 \\
\hline 021 & Chilli-Fallow-T. Aman & 52995 & 0.619 & 45 & 146 \\
\hline 022 & Lentil-Jute-T. Aman & 51875 & 0.606 & 34 & 96 \\
\hline 023 & Vegetable-Vegetable-T. Aman & 51745 & 0.604 & 49 & 127 \\
\hline
\end{tabular}


Table 2. Continued.

\begin{tabular}{|c|c|c|c|c|c|}
\hline & Cropping pattern & Area (ha) & $\%$ of NCA & No. of district & No. of upazila \\
\hline 024 & Wheat-Jute-Fallow & 48700 & 0.568 & 32 & 82 \\
\hline 025 & Potato-Maize-T. Aman & 47690 & 0.557 & 19 & 68 \\
\hline 026 & Boro-Aus-Fallow & 45865 & 0.535 & 34 & 71 \\
\hline 027 & Onion-Jute-Fallow & 45200 & 0.528 & 29 & 67 \\
\hline 028 & Boro-Jute-T. Aman & 40440 & 0.472 & 19 & 56 \\
\hline 029 & Felon-Fallow-T. Aman & 37675 & 0.440 & 14 & 53 \\
\hline 030 & Mustard-Boro-B.Aman & 36520 & 0.426 & 14 & 32 \\
\hline 031 & Wheat-Aus-T. Aman & 36425 & 0.425 & 35 & 93 \\
\hline 032 & Mustard-Jute-T. Aman & 32740 & 0.382 & 29 & 64 \\
\hline 033 & Lentil-Jute-Fallow & 32360 & 0.378 & 27 & 67 \\
\hline 034 & Boro-Fish & 31400 & 0.367 & 3 & 17 \\
\hline 035 & Vegetable-Fallow-Fallow & 31115 & 0.363 & 35 & 84 \\
\hline 036 & Groundnut-Fallow-T. Aman & 29210 & 0.341 & 23 & 60 \\
\hline 037 & Vegetable-Aus-T. Aman & 28700 & 0.335 & 42 & 87 \\
\hline 038 & Water Melon-Fallow-T. Aman & 28340 & 0.331 & 17 & 49 \\
\hline 039 & Potato-Jute-T. Aman & 28310 & 0.330 & 36 & 95 \\
\hline 040 & Potato-B.Aman & 23900 & 0.279 & 9 & 25 \\
\hline 041 & Soybean-Fallow-T. Aman & 23670 & 0.276 & 5 & 10 \\
\hline 042 & Soybean-Aus-T. Aman & 23170 & 0.270 & 5 & 8 \\
\hline 043 & Potato-Fallow-T. Aman & 22715 & 0.265 & 34 & 108 \\
\hline 044 & Potato-Aus-T. Aman & 22405 & 0.262 & 29 & 79 \\
\hline 045 & Wheat-Mungbean-T. Aman & 21760 & 0.254 & 20 & 50 \\
\hline 046 & Grasspea-B.Aman & 21480 & 0.251 & 21 & 52 \\
\hline 047 & Maize-Jute-Fallow & 21425 & 0.250 & 19 & 33 \\
\hline 048 & Maize-Jute-T. Aman & 21325 & 0.249 & 18 & 38 \\
\hline 049 & Vegetable-Boro-T. Aman & 21220 & 0.248 & 22 & 42 \\
\hline 050 & Grasspea-Jute-Fallow & 21000 & 0.245 & 21 & 39 \\
\hline 051 & Fish-T. Aman & 20400 & 0.238 & 3 & 8 \\
\hline 052 & Soybean-Jute-T. Aman & 20000 & 0.233 & 4 & 6 \\
\hline 053 & Potato-Boro-Fallow & 19360 & 0.226 & 17 & 27 \\
\hline 054 & Mustard-Boro-Aus & 18140 & 0.212 & 10 & 16 \\
\hline 055 & Mustard-B.Aman & 17210 & 0.201 & 12 & 24 \\
\hline 056 & Mustard-Jute-Fallow & 17180 & 0.201 & 16 & 35 \\
\hline 057 & Sweet Potato-Fallow-T. Aman & 16990 & 0.198 & 31 & 103 \\
\hline 058 & Groundnut-Fallow-Fallow & 16485 & 0.192 & 35 & 76 \\
\hline 059 & Boro-Jute-Fallow & 16370 & 0.191 & 19 & 25 \\
\hline 060 & Fallow-B.Aman & 16360 & 0.191 & 15 & 29 \\
\hline 061 & Wheat-Maize-T. Aman & 16320 & 0.191 & 9 & 14 \\
\hline 062 & Wheat-Aus-Fallow & 16200 & 0.189 & 17 & 26 \\
\hline 063 & Vegetable-Aus-Fallow & 15940 & 0.186 & 23 & 41 \\
\hline 064 & Tobacco-Jute-T. Aman & 15200 & 0.177 & 9 & 19 \\
\hline 065 & Mustard-Fallow-T. Aman & 14870 & 0.174 & 33 & 84 \\
\hline 066 & Groundnut- Aus-T. Aman & 14535 & 0.170 & 10 & 18 \\
\hline 067 & Potato-Jute-Fallow & 14515 & 0.169 & 25 & 53 \\
\hline 068 & Boro-Sesbania-T. Aman & 14490 & 0.169 & 20 & 33 \\
\hline 069 & Chilli-Aus-T. Aman & 14240 & 0.166 & 21 & 38 \\
\hline 070 & Wheat-B.Aman & 13835 & 0.162 & 16 & 32 \\
\hline
\end{tabular}


Table 2. Continued.

\begin{tabular}{|c|c|c|c|c|c|}
\hline & Cropping pattern & Area (ha) & $\%$ of NCA & No. of district & No. of upazila \\
\hline 071 & Mustard-Aus-T. Aman & 13790 & 0.161 & 30 & 52 \\
\hline 072 & Fallow-Jute-T. Aman & 13620 & 0.159 & 18 & 32 \\
\hline 073 & Maize-Aus-Fallow & 13615 & 0.159 & 14 & 18 \\
\hline 074 & Garlic-Jute-Fallow & 13475 & 0.157 & 26 & 48 \\
\hline 075 & Boro-Vegetable (Float/Norm) & 13335 & 0.156 & 27 & 36 \\
\hline 076 & Boro-Fallow-Blackgram & 13270 & 0.155 & 27 & 44 \\
\hline 077 & Chilli-Jute-Fallow & 13260 & 0.155 & 22 & 54 \\
\hline 078 & Vegetable-Jute-Fallow & 13185 & 0.154 & 26 & 47 \\
\hline 079 & Garlic-Jute-T. Aman & 13090 & 0.153 & 38 & 78 \\
\hline 080 & Wheat-Aus-Blackgram & 13000 & 0.152 & 6 & 8 \\
\hline 081 & Maize-Fallow-Fallow & 12920 & 0.151 & 22 & 38 \\
\hline 082 & Lentil-Fallow-T. Aman & 12680 & 0.148 & 29 & 68 \\
\hline 083 & Potato-Vegetable-T. Aman & 12620 & 0.147 & 30 & 59 \\
\hline 084 & Lentil-Sesame-T. Aman & 12450 & 0.145 & 14 & 33 \\
\hline 085 & Fallow-Aus+Non-rice (jhum) & 11900 & 0.139 & 3 & 20 \\
\hline 086 & Onion-Vegtable-Vegetable & 11735 & 0.137 & 39 & 87 \\
\hline 087 & Fallow-Sesame-T. Aman & 11640 & 0.136 & 13 & 20 \\
\hline 088 & Vegetable-Jute-T. Aman & 11305 & 0.132 & 25 & 51 \\
\hline 089 & Lentil-Aus-T. Aman & 11255 & 0.131 & 25 & 46 \\
\hline 090 & Onion-B.Aman & 10905 & 0.127 & 14 & 27 \\
\hline 091 & Garlic-B.Aman & 10850 & 0.127 & 15 & 21 \\
\hline 092 & Potato-Sesbania & 10770 & 0.126 & 5 & 15 \\
\hline 093 & Wheat-Sesame-T. Aman & 10650 & 0.124 & 15 & 33 \\
\hline 094 & Water Melon-Aus-T. Aman & 10355 & 0.121 & 11 & 14 \\
\hline 095 & Grasspea-Jute-T. Aman & 10300 & 0.120 & 25 & 43 \\
\hline 096 & Potato-Maize-Fallow & 9640 & 0.113 & 10 & 16 \\
\hline 097 & Chilli-Fallow-Fallow & 9265 & 0.108 & 40 & 66 \\
\hline 098 & Sesame-Fallow-T. Aman & 9265 & 0.108 & 19 & 31 \\
\hline 099 & Felon-Aus-T. Aman & 9245 & 0.108 & 7 & 16 \\
\hline 100 & Boro-Sesbania-Fallow & 9180 & 0.107 & 12 & 19 \\
\hline 101 & Chilli-B.Aman & 9120 & 0.106 & 10 & 23 \\
\hline 102 & Chilli-Vegetable-Fallow & 8810 & 0.103 & 44 & 104 \\
\hline 103 & Onion-Aus-Fallow & 8705 & 0.102 & 12 & 17 \\
\hline 104 & Fallow-Fallow-Blackgram & 8695 & 0.102 & 34 & 59 \\
\hline 105 & Boro-Vegetable-T. Aman & 8660 & 0.101 & 9 & 16 \\
\hline 106 & Boro-Aus-Blackgram & 8540 & 0.100 & 5 & 5 \\
\hline 107 & Sweet Potato-Fallow-Fallow & 7980 & 0.093 & 37 & 98 \\
\hline 108 & Mustard-Boro-Aus-T. Aman & 7850 & 0.092 & 14 & 30 \\
\hline 109 & Blackgram-B.Aman & 7625 & 0.089 & 11 & 24 \\
\hline 110 & Chilli-Aus-Fallow & 7565 & 0.088 & 14 & 27 \\
\hline 111 & Tobacco-Maize-T. Aman & 7470 & 0.087 & 5 & 12 \\
\hline 112 & Potato-Boro-Aus & 7420 & 0.087 & 4 & 6 \\
\hline 113 & Coriander-Jute-Fallow & 7390 & 0.086 & 15 & 25 \\
\hline 114 & Chilli-Jute-T. Aman & 7250 & 0.085 & 20 & 35 \\
\hline 115 & Fallow-Vegetable-T. Aman & 7130 & 0.083 & 16 & 29 \\
\hline 116 & Maize-Vegetable-Fallow & 7120 & 0.083 & 14 & 19 \\
\hline 117 & Vegetable-Boro-Fallow & 7100 & 0.083 & 9 & 10 \\
\hline
\end{tabular}


Table 2. Continued.

\begin{tabular}{|c|c|c|c|c|c|}
\hline & Cropping pattern & Area (ha) & $\%$ of NCA & No. of district & No. of upazila \\
\hline 118 & Lentil-Mungbean-T. Aman & 6955 & 0.081 & 11 & 22 \\
\hline 119 & Lentil-B.Aman & 6550 & 0.076 & 16 & 30 \\
\hline 120 & Onion-Fallow-T. Aman & 6380 & 0.074 & 32 & 64 \\
\hline 121 & Lentil-Vegetable-Vegetable & 6330 & 0.074 & 19 & 30 \\
\hline 122 & Mustard-Maize-T. Aman & 6310 & 0.074 & 9 & 19 \\
\hline 123 & Wheat-Vegetable-Vegetable & 6100 & 0.071 & 16 & 21 \\
\hline 124 & Tobacco-Aus-T. Aman & 6040 & 0.071 & 5 & 12 \\
\hline 125 & Soybean-B.Aman & 5650 & 0.066 & 4 & 4 \\
\hline 126 & Boro-Fallow-T. Aman +Fish & 5410 & 0.063 & 3 & 7 \\
\hline 127 & Tobacco-Fallow-T. Aman & 5310 & 0.062 & 7 & 15 \\
\hline 128 & Wheat-Vegetable-T. Aman & 5205 & 0.061 & 15 & 25 \\
\hline 129 & Vegetable-B.Aman & 5200 & 0.061 & 9 & 17 \\
\hline 130 & Maize-B.Aman & 5030 & 0.059 & 10 & 15 \\
\hline 131 & Mustard-Aus-Fallow & 4935 & 0.058 & 15 & 22 \\
\hline 132 & Maize-Mungbean-T. Aman & 4900 & 0.057 & 1 & 3 \\
\hline 133 & Mustard-Mungbean-T. Aman & 4710 & 0.055 & 12 & 18 \\
\hline 134 & Chickpea-Fallow-T. Aman & 4700 & 0.055 & 12 & 32 \\
\hline 135 & Grasspea-Boro-Fallow & 4690 & 0.055 & 8 & 12 \\
\hline 136 & Coriander-Jute-T. Aman & 4625 & 0.054 & 17 & 30 \\
\hline 137 & Lentil-Aus-Fallow & 4570 & 0.053 & 11 & 15 \\
\hline 138 & Vegetable-Maize-T. Aman & 4500 & 0.053 & 9 & 15 \\
\hline 139 & Garlic-Vegetable-Vegetable & 4385 & 0.051 & 27 & 68 \\
\hline 140 & Potato-Maize-Aus & 4300 & 0.050 & 3 & 4 \\
\hline 141 & Onion-Aus-T. Aman & 4260 & 0.050 & 17 & 26 \\
\hline 142 & Tobacco-Jute-Fallow & 4050 & 0.047 & 4 & 8 \\
\hline 143 & Wheat-Jute-Blackgram & 3910 & 0.046 & 7 & 9 \\
\hline 144 & Wheat-Fallow-Fallow & 3810 & 0.044 & 6 & 8 \\
\hline 145 & Wheat-Chilli-Fallow & 3780 & 0.044 & 7 & 9 \\
\hline 146 & Maize-Maize-Fallow & 3720 & 0.043 & 3 & 5 \\
\hline 147 & Wheat-Fallow-T. Aman (Orchard) & 3700 & 0.043 & 1 & 2 \\
\hline 148 & Garlic-Fallow-T. Aman & 3680 & 0.043 & 31 & 59 \\
\hline 149 & Lentil-Sesame-Fallow & 3680 & 0.043 & 15 & 20 \\
\hline 150 & Chilli-Boro-Jute & 3600 & 0.042 & 2 & 3 \\
\hline 151 & Maize-Fallow-Blackgram & 3600 & 0.042 & 4 & 4 \\
\hline 152 & Vegetable-Fallow-Blackgram & 3525 & 0.041 & 16 & 33 \\
\hline 153 & Fallow-B.Aus+B.Aman & 3470 & 0.041 & 3 & 5 \\
\hline 154 & Sweet Potato-Jute-Fallow & 3380 & 0.039 & 13 & 21 \\
\hline 155 & Mustard-Sesame-T. Aman & 3360 & 0.039 & 12 & 15 \\
\hline 156 & Vegetable-Onion-Aus & 3200 & 0.037 & 3 & 3 \\
\hline 157 & Tobacco-Aus-Fallow & 3180 & 0.037 & 5 & 10 \\
\hline 158 & Coriander-Fallow-Fallow & 3175 & 0.037 & 41 & 64 \\
\hline 159 & Potato-Boro-Aus-T. Aman & 3140 & 0.037 & 5 & 9 \\
\hline 160 & Onion-Sesame-T. Aman & 3080 & 0.036 & 4 & 6 \\
\hline 161 & Maize-Maize-T. Aman & 3070 & 0.036 & 6 & 8 \\
\hline 162 & Wheat-Maize-Fallow & 3070 & 0.036 & 2 & 4 \\
\hline 163 & Sesame-Fallow-Blackgram & 3060 & 0.036 & 6 & 10 \\
\hline 164 & Maize-Aus-Vegetable & 3000 & 0.035 & 3 & 4 \\
\hline
\end{tabular}


Table 2. Continued.

\begin{tabular}{|c|c|c|c|c|c|}
\hline & Cropping pattern & Area (ha) & $\%$ of NCA & No. of district & No. of upazila \\
\hline 165 & Mustard-Boro-Jute & 3000 & 0.035 & 5 & 7 \\
\hline 166 & Mustard-Boro-Jute-T. Aman & 2980 & 0.035 & 7 & 13 \\
\hline 167 & Maize-Aus-T. Aman & 2970 & 0.035 & 17 & 27 \\
\hline 168 & Lentil-Maize-T. Aman & 2920 & 0.034 & 4 & 8 \\
\hline 169 & Wheat-Mungbean-Fallow & 2875 & 0.034 & 5 & 7 \\
\hline 170 & Groundnut-B.Aman & 2820 & 0.033 & 5 & 7 \\
\hline 171 & Lentil-Fallow-Fallow(Orchard) & 2780 & 0.032 & 3 & 5 \\
\hline 172 & Fallow-Sesame+B.Aman & 2750 & 0.032 & 5 & 7 \\
\hline 173 & Potato-Groundnut-T. Aman & 2720 & 0.032 & 3 & 5 \\
\hline 174 & Soybean-Fallow-Fallow & 2710 & 0.032 & 3 & 4 \\
\hline 175 & Grasspea-Boro-B.Aman & 2700 & 0.032 & 3 & 3 \\
\hline 176 & Lentil-Vegetable-T. Aman & 2660 & 0.031 & 11 & 11 \\
\hline 177 & Vegetable-Groundnut-T. Aman & 2660 & 0.031 & 4 & 5 \\
\hline 178 & Pea-B.Aman & 2620 & 0.031 & 11 & 16 \\
\hline 179 & Garlic-Mungbean-T. Aman & 2500 & 0.029 & 1 & 2 \\
\hline 180 & Garlic-Aus-T. Aman & 2400 & 0.028 & 13 & 20 \\
\hline 181 & Millet(Kaon)-Fallow-T. Aman & 2390 & 0.028 & 6 & 6 \\
\hline 182 & Muskmelon-Fallow-T. Aman & 2310 & 0.027 & 10 & 23 \\
\hline 183 & Potato-Aus-Fallow & 2310 & 0.027 & 13 & 16 \\
\hline 184 & Tobacco-Boro-T. Aman & 2300 & 0.027 & 3 & 4 \\
\hline 185 & Blackgram-Jute-Fallow & 2295 & 0.027 & 12 & 16 \\
\hline 186 & Grasspea-Fallow-Fallow & 2295 & 0.027 & 9 & 11 \\
\hline 187 & Fallow-Fallow-T. Aman +Fish & 2200 & 0.026 & 2 & 2 \\
\hline 188 & Potato-Maize-Vegetable & 2190 & 0.026 & 4 & 4 \\
\hline 189 & Coriander-Fallow-T. Aman & 2185 & 0.026 & 31 & 62 \\
\hline 190 & Vegetable-Maize-Fallow & 2180 & 0.025 & 6 & 7 \\
\hline 191 & Potato-Boro-Jute-T. Aman & 2160 & 0.025 & 4 & 5 \\
\hline 192 & Sweet Potato-Aus-T. Aman & 2150 & 0.025 & 2 & 2 \\
\hline 193 & Wheat-Mung-Fallow(Orchard) & 2150 & 0.025 & 2 & 3 \\
\hline 194 & Onion-Sesame+B.Aman & 2100 & 0.025 & 2 & 3 \\
\hline 195 & Pea-Jute-Fallow & 2100 & 0.025 & 10 & 15 \\
\hline 196 & Sunflower-Fallow-T. Aman & 2100 & 0.025 & 7 & 23 \\
\hline 197 & Coriander-Vegetable-Fallow & 2090 & 0.024 & 20 & 29 \\
\hline 198 & Potato-Chilli-Fallow & 2070 & 0.024 & 10 & 17 \\
\hline 199 & Potato-Sesame-T. Aman & 2060 & 0.024 & 15 & 23 \\
\hline 200 & Blackgram(Fodder)-Boro-B.Aman & 2000 & 0.023 & 1 & 1 \\
\hline 201 & Wheat-Maize-Vegetable & 2000 & 0.023 & 2 & 2 \\
\hline 202 & Pea-Fallow-T. Aman & 1950 & 0.023 & 18 & 27 \\
\hline 203 & Potato-Sesame-Fallow & 1930 & 0.023 & 6 & 10 \\
\hline 204 & Blackgram-Jute-T. Aman & 1900 & 0.022 & 11 & 14 \\
\hline 205 & Mustard-Aus-Blackgram & 1900 & 0.022 & 2 & 2 \\
\hline 206 & Mustard-Jute-Vegetable & 1900 & 0.022 & 3 & 5 \\
\hline 207 & Wheat-Aus-Onion & 1900 & 0.022 & 1 & 1 \\
\hline 208 & Blackcumin-Jute-Fallow & 1840 & 0.021 & 6 & 12 \\
\hline 209 & Maize-Sesame-T. Aman & 1835 & 0.021 & 6 & 9 \\
\hline 210 & Maize-Vegetable-T. Aman & 1810 & 0.021 & 11 & 12 \\
\hline 211 & Millet(Kaon)+Sesame-Fallow & 1810 & 0.021 & 5 & 7 \\
\hline
\end{tabular}


Table 2. Continued.

\begin{tabular}{|c|c|c|c|c|c|}
\hline & Cropping pattern & Area (ha) & $\%$ of NCA & No. of district & No. of upazila \\
\hline 212 & Onion-Aus-Blackgram & 1810 & 0.021 & 5 & 6 \\
\hline 213 & Grasspea-Boro-Aus & 1800 & 0.021 & 1 & 1 \\
\hline 214 & Grasspea-Sesame+B.Aman & 1800 & 0.021 & 2 & 2 \\
\hline 215 & Grasspea-Sesame-T. Aman & 1790 & 0.021 & 5 & 6 \\
\hline 216 & Grasspea-Sesbania-Fallow & 1780 & 0.021 & 4 & 4 \\
\hline 217 & Mustard-B.Aus+B.Aman & 1780 & 0.021 & 4 & 4 \\
\hline 218 & Blackgram-Aus-T. Aman & 1735 & 0.020 & 10 & 13 \\
\hline 219 & Mustard-Sesame+B.Aman & 1650 & 0.019 & 3 & 4 \\
\hline 220 & Tobacco-Sesbania-T. Aman & 1620 & 0.019 & 1 & 2 \\
\hline 221 & Vegetable-Aus-Blackgram & 1610 & 0.019 & 4 & 4 \\
\hline 222 & Grasspea-B.Aus+B.Aman & 1580 & 0.018 & 6 & 8 \\
\hline 223 & Maize-Sesame-Fallow & 1580 & 0.018 & 6 & 8 \\
\hline 224 & Groundnut- Aus-Fallow & 1550 & 0.018 & 5 & 5 \\
\hline 225 & Wheat-Sesame-Fallow & 1550 & 0.018 & 7 & 8 \\
\hline 226 & Groundnut-Jute-T. Aman & 1530 & 0.018 & 7 & 13 \\
\hline 227 & Boro-Fallow-Maize & 1500 & 0.018 & 1 & 1 \\
\hline 228 & Tobacco-Vegetable-Vegetable & 1500 & 0.018 & 1 & 1 \\
\hline 229 & Onion-Maize-T. Aman & 1490 & 0.017 & 7 & 9 \\
\hline 230 & Coriander-B.Aman & 1480 & 0.017 & 11 & 11 \\
\hline 231 & Garlic-Aus-Fallow & 1450 & 0.017 & 12 & 15 \\
\hline 232 & Vegetable-Jute-Vegetable & 1450 & 0.017 & 5 & 5 \\
\hline 233 & Chilli-Vegetable-T. Aman & 1405 & 0.016 & 15 & 19 \\
\hline 234 & Fallow-Aus-Fallow & 1400 & 0.016 & 3 & 3 \\
\hline 235 & Maize-Boro-T. Aman & 1400 & 0.016 & 2 & 2 \\
\hline 236 & Maize-Aus-Blackgram & 1370 & 0.016 & 3 & 3 \\
\hline 237 & Cotton-Aus-Fallow & 1330 & 0.016 & 3 & 3 \\
\hline 238 & Grasspea-Sesame-Fallow & 1330 & 0.016 & 7 & 10 \\
\hline 239 & Groundnut-Fallow-Blackgram & 1320 & 0.015 & 3 & 3 \\
\hline 240 & Wheat-Jute-Vegetable & 1320 & 0.015 & 6 & 6 \\
\hline 241 & Pea-Aus-Vegetable & 1310 & 0.015 & 7 & 8 \\
\hline 242 & Onion-Sesame-Fallow & 1270 & 0.015 & 8 & 9 \\
\hline 243 & Blackcumin-Jute-T. Aman & 1260 & 0.015 & 6 & 11 \\
\hline 244 & Sesame-Aus-Fallow & 1190 & 0.014 & 3 & 4 \\
\hline 245 & Sesame-Aus-T. Aman & 1180 & 0.014 & 4 & 5 \\
\hline 246 & Water Melon-Fallow-Fallow & 1165 & 0.014 & 9 & 12 \\
\hline 247 & Pea-Vegetable-Fallow & 1160 & 0.014 & 4 & 5 \\
\hline 248 & Potato-Mungbean-T. Aman & 1140 & 0.013 & 11 & 13 \\
\hline 249 & Potato-Groundnut & 1110 & 0.013 & 5 & 8 \\
\hline 250 & Tobacco-Fallow-Fallow & 1045 & 0.012 & 5 & 8 \\
\hline 251 & Potato-Maize-Aus-Vegetable & 1030 & 0.012 & 3 & 3 \\
\hline 252 & Fallow-Fallow-T. Aman (Orchard) & 1000 & 0.012 & 1 & 1 \\
\hline 253 & Maize-Sesbania-Fallow & 1000 & 0.012 & 1 & 1 \\
\hline 254 & Lentil-Sesame+B.Aman & 980 & 0.011 & 1 & 1 \\
\hline 255 & Maize-Mungbean-Vegetable & 950 & 0.011 & 3 & 3 \\
\hline 256 & Millet (Cheena)-Fallow-Fallow & 935 & 0.011 & 5 & 6 \\
\hline 257 & Potato-Sweet gourd-Aus & 910 & 0.011 & 6 & 8 \\
\hline 258 & Wheat-Fallow-Blackgram & 900 & 0.011 & 1 & 1 \\
\hline
\end{tabular}


Table 2. Continued.

\begin{tabular}{|c|c|c|c|c|c|}
\hline & Cropping pattern & Area (ha) & $\%$ of NCA & No. of district & No. of upazila \\
\hline 259 & Mustard-Fallow-Fallow & 895 & 0.010 & 9 & 13 \\
\hline 260 & Coriander-Sesame-T. Aman & 845 & 0.010 & 5 & 5 \\
\hline 261 & Vegetable-Boro-Aus-T. Aman & 820 & 0.010 & 4 & 5 \\
\hline 262 & Vegetable-Boro-Jute & 800 & 0.009 & 2 & 3 \\
\hline 263 & Potato+Sweetgourd-Aus-T. Aman & 780 & 0.009 & 3 & 5 \\
\hline 264 & Wheat-Sesame-Blackgram(Orchard) & 760 & 0.009 & 3 & 3 \\
\hline 265 & Sweet Potato-B.Aman & 740 & 0.009 & 6 & 6 \\
\hline 266 & Mustard-Boro-Sesbania & 700 & 0.008 & 2 & 2 \\
\hline 267 & Potato-Boro-Vegetable & 700 & 0.008 & 4 & 4 \\
\hline 268 & Soybean-Aus-Fallow & 700 & 0.008 & 1 & 1 \\
\hline 269 & Sweet Potato-Jute-T. Aman & 675 & 0.008 & 10 & 13 \\
\hline 270 & Maize-Groundnut & 620 & 0.007 & 1 & 1 \\
\hline 271 & Millet(Cheena)-Jute-Fallow & 620 & 0.007 & 2 & 2 \\
\hline 272 & Sesame+B.Aman-Blackgram & 600 & 0.007 & 1 & 1 \\
\hline 273 & Tobacco-Maize-Vegetable & 600 & 0.007 & 1 & 1 \\
\hline 274 & Tobacco-Sesbania & 600 & 0.007 & 1 & 1 \\
\hline 275 & Wheat-Aus-Cotton & 600 & 0.007 & 1 & 1 \\
\hline 276 & Groundnut-Sesame-Fallow & 590 & 0.007 & 4 & 5 \\
\hline 277 & Potato-Boro-Jute & 590 & 0.007 & 2 & 2 \\
\hline 278 & Wheat-Ginger/Turmeric & 580 & 0.007 & 2 & 2 \\
\hline 279 & Sweet Potato-Vegetable-Fallow & 540 & 0.006 & 5 & 9 \\
\hline 280 & Water Melon-B.Aman & 535 & 0.006 & 3 & 3 \\
\hline 281 & Garlic-Fallow-Fallow & 530 & 0.006 & 10 & 14 \\
\hline 282 & Onion-Maize-Fallow & 520 & 0.006 & 4 & 4 \\
\hline 283 & Potato-Onion-T. Aman & 510 & 0.006 & 2 & 2 \\
\hline 284 & Felon-Aus-Fallow & 470 & 0.005 & 2 & 3 \\
\hline 285 & Grasspea-Mungbean-T. Aman & 440 & 0.005 & 5 & 5 \\
\hline 286 & Boro-Maize-Fallow & 410 & 0.005 & 2 & 2 \\
\hline 287 & Potato+Maize-B.Aman & 410 & 0.005 & 2 & 3 \\
\hline 288 & Felon-Fallow-Fallow & 380 & 0.004 & 3 & 4 \\
\hline 289 & Groundnut-Millet(Kaon)-Fallow & 380 & 0.004 & 2 & 2 \\
\hline 290 & Chickpea-Aus-T. Aman & 375 & 0.004 & 5 & 7 \\
\hline 291 & Potato-Sesame-Aus & 360 & 0.004 & 4 & 4 \\
\hline 292 & Boro-Chilli-Fallow & 350 & 0.004 & 1 & 1 \\
\hline 293 & Boro-Maize-Blackgram & 350 & 0.004 & 1 & 1 \\
\hline 294 & Sesame-B.Aman & 330 & 0.004 & 2 & 2 \\
\hline 295 & Garlic+Muskmelon-B.Aman & 320 & 0.004 & 1 & 2 \\
\hline 296 & Garlic+WaterMelon-B.Aman & 320 & 0.004 & 1 & 2 \\
\hline 297 & Mungbean-Jute-Fallow & 300 & 0.004 & 6 & 8 \\
\hline 298 & Sesame-Jute-T. Aman & 290 & 0.003 & 2 & 2 \\
\hline 299 & Chickpea-Jute-T. Aman & 270 & 0.003 & 7 & 11 \\
\hline 300 & Grasspea-Aus-Blackgram & 250 & 0.003 & 1 & 1 \\
\hline 301 & Musk Melon-B.Aman & 215 & 0.003 & 2 & 5 \\
\hline 302 & Potato+Maize-Vegetable-T. Aman & 210 & 0.002 & 2 & 2 \\
\hline 303 & Mustard-Maize-Jute & 200 & 0.002 & 1 & 1 \\
\hline 304 & Vegetab-Onion-Jute-T. Aman & 200 & 0.002 & 1 & 1 \\
\hline 305 & Mungbean-Jute-T. Aman & 180 & 0.002 & 9 & 11 \\
\hline
\end{tabular}


Table 2. Continued.

\begin{tabular}{|c|c|c|c|c|c|}
\hline & Cropping pattern & Area (ha) & $\%$ of NCA & No. of district & No. of upazila \\
\hline 306 & Soybean-Jute-Fallow & 140 & 0.002 & 2 & 2 \\
\hline 307 & Sunflower-Jute-Fallow & 135 & 0.002 & 3 & 3 \\
\hline 308 & Mungbean-B.Aus+B.Aman & 130 & 0.002 & 4 & 4 \\
\hline 309 & Musk Melon-Fallow-Fallow & 125 & 0.001 & 5 & 5 \\
\hline 310 & Lentil-Mungbean-Jute-T. Aman & 100 & 0.001 & 1 & 1 \\
\hline 311 & Mustard-Mungbean-Aus-T. Aman & 100 & 0.001 & 1 & 1 \\
\hline 312 & Potato+S. gourd-M.bean-T. Aman & 80 & 0.001 & 1 & 1 \\
\hline 313 & Tobacco-Mungbean-T. Aman & 50 & 0.001 & 1 & 1 \\
\hline 314 & Tobacco-Mungbean-Vegetable & 40 & 0.000 & 1 & 1 \\
\hline 315 & Mungbean-Aus-Fallow & 20 & 0.000 & 1 & 1 \\
\hline \multirow[t]{4}{*}{316} & Barley-Fallow-Fallow & 15 & 0.000 & 1 & 2 \\
\hline & Others & 64790 & 0.756 & 64 & 486 \\
\hline & Annual crops & 243765 & 2.846 & 64 & 486 \\
\hline & Net cropped area of Bangladesh & 8566500 & 100.000 & 64 & 486 \\
\hline
\end{tabular}

In Bangladesh, 316 CPs were found which deserved to be included in the $\mathrm{CP}$ list of the study excluding the minor ones (Table 2). BoroFallow-T. Aman was the most dominant CP which occupied $26.92 \%$ of the NCA. Whereas the last CP was the Barley-Fallow-Fallow which occupied only $0.0002 \%$ of the NCA. There had been many more CPs, whose hectarages were quite low, were not considered in the list. Those CPs were kept in 'Other' category where only summation of all of their hectarages was considered. The most dominant $\mathrm{CP}$, Boro-Fallow-T. Aman occupied significantly higher percentage of NCA than that of its next following $\mathrm{CP}$, Boro-Fallow-Fallow which occupied less than half of the percentage of NCA of Boro-Fallow-T. Aman. The next three CPs were Fallow-Fallow-T. Aman, Boro-Aus-T. Aman and Fallow-Aus-T. Aman, respectively. These five CPs occupied $50.86 \%$ of the NCA. Interestingly the results unveiled that in these foremost five CPs, no other crop than rice was included. It indicated the dominancy of rice culture in Bangladesh. Sujatha et al. (2011) found similar dominancy of rice culture in Tamil Nadu in the neighbouring country, India. Boro and T. Aman contribute the large share in crop production. In T. Aman season, monsoon rain is plenty, however it is usually unreliable whereas in Boro season irrigation is comparatively reliable. Availability of water in these two seasons brought more area under rice cultivation. Hence Boro-Fallow-T. Aman became the most dominant $\mathrm{CP}$ of the country. T. Aman is usually cultivated in medium highland. Therefore, this $\mathrm{CP}$ is practiced in medium highland of the country. All other CPs including 'Other' category, that could be few hundreds, constituted the rest $49 \%$ of NCA. In Table 2, 'number of upazila' indicates the existence of a specific $\mathrm{CP}$ in total number of upazilas. The most dominant $\mathrm{CP}$, BoroFallow-T. Aman was present in 426 upazilas out of 486 . It was found that higher the percentage of NCA occupied by a CP greater the presence of the $\mathrm{CP}$ in different upazilas. In terms of area coverage some of the CPs mentioned in this study were very minor CPs, such as BoroFallow-Maize, Maize-Sesbania-Fallow etc. These two CPs were present in one upazila only with low area coverage.

In the earlier table, all the CPs were listed, however, in Table 3, CPs with rice exclusively was separated. There were 17 CPs containing exclusively rice crops. Foremost five of them were most dominant CPs, mentioned in earlier para. Twelve others may be considered as minor. Among these minors, in some CPs fishes were included in rainy season. In another $\mathrm{CP}$, single $\mathrm{T}$. Aman was cultivated in the orchard where fruit trees were still small. All these 17 CPs occupied $54.77 \%$ of the NCA. Thus rice exclusive CPs 
Table 3. List of cropping patterns with rice exclusively and area coverage, 2014-15.

\begin{tabular}{|c|c|c|c|c|c|}
\hline & Cropping pattern & Area (ha) & $\%$ of NCA & No. of district & No. of upazila \\
\hline 01 & Boro-Fallow-T. Aman & 2306005 & 26.92 & 63 & 426 \\
\hline 02 & Boro-Fallow-Fallow & 1139530 & 13.30 & 59 & 342 \\
\hline 03 & Fallow-Fallow-T. Aman & 509480 & 5.95 & 36 & 162 \\
\hline 04 & Boro-Aus-T. Aman & 209015 & 2.44 & 47 & 177 \\
\hline 05 & Fallow-Aus-T. Aman & 193275 & 2.26 & 30 & 108 \\
\hline 06 & Boro-B.Aman & 183070 & 2.14 & 32 & 113 \\
\hline 07 & Boro-Aus-Fallow & 45865 & 0.54 & 34 & 71 \\
\hline 08 & Boro-Fish & 31400 & 0.37 & 3 & 17 \\
\hline 09 & Fish-T. Aman & 20400 & 0.24 & 3 & 8 \\
\hline 10 & Fallow-B.Aman & 16360 & 0.19 & 15 & 29 \\
\hline 11 & Boro-Sesbania-T. Aman & 14490 & 0.17 & 20 & 33 \\
\hline 12 & Boro-Sesbania-Fallow & 9180 & 0.11 & 12 & 19 \\
\hline 13 & Boro-Fallow-T. Aman +Fish & 5410 & 0.06 & 3 & 7 \\
\hline 14 & Fallow-B.Aus+B.Aman & 3470 & 0.04 & 3 & 5 \\
\hline 15 & Fallow-Fallow-T. Aman +Fish & 2200 & 0.03 & 2 & 2 \\
\hline 16 & Fallow-Aus-Fallow & 1400 & 0.02 & 3 & 3 \\
\hline \multirow[t]{2}{*}{17} & F-F-T. Aman (Orchard) & 1000 & 0.01 & 1 & 1 \\
\hline & Total of exclusive rice area & 4691550 & 54.77 & - & - \\
\hline
\end{tabular}

Table 4. List of cropping patterns with Boro crop and area coverage, 2014-15.

\begin{tabular}{|c|c|c|c|c|c|}
\hline & Cropping pattern & Area (ha) & $\%$ of NCA & No. of district & No. of upazila \\
\hline 01 & Boro-Fallow-T. Aman & 2306005 & 26.92 & 63 & 426 \\
\hline 02 & Boro-Fallow-Fallow & 1139530 & 13.30 & 59 & 342 \\
\hline 03 & Boro-Aus-T. Aman & 209015 & 2.44 & 47 & 177 \\
\hline 04 & Mustard-Boro-T. Aman & 184620 & 2.16 & 51 & 203 \\
\hline 05 & Boro-B.Aman & 183070 & 2.14 & 32 & 113 \\
\hline 06 & Potato-Boro-T. Aman & 180380 & 2.11 & 33 & 115 \\
\hline 07 & Mustard-Boro-Fallow & 143130 & 1.67 & 37 & 112 \\
\hline 08 & Boro-Aus-Fallow & 45865 & 0.54 & 34 & 71 \\
\hline 09 & Boro-Jute-T. Aman & 40440 & 0.47 & 19 & 56 \\
\hline 10 & Mustard-Boro-B.Aman & 36520 & 0.43 & 14 & 32 \\
\hline 11 & Boro-Fish & 31400 & 0.37 & 3 & 17 \\
\hline 12 & Vegetable-Boro-T. Aman & 21220 & 0.25 & 22 & 42 \\
\hline 13 & Potato-Boro-Fallow & 19360 & 0.23 & 17 & 27 \\
\hline 14 & Mustard-Boro-Aus & 18140 & 0.21 & 10 & 16 \\
\hline 15 & Boro-Jute-Fallow & 16370 & 0.19 & 19 & 25 \\
\hline 16 & Boro-Sesbania-T. Aman & 14490 & 0.17 & 20 & 33 \\
\hline 17 & Boro-Vegetable (Float/Norm) & 13335 & 0.16 & 27 & 36 \\
\hline 18 & Boro-Fallow-Blackgram & 13270 & 0.15 & 27 & 44 \\
\hline 19 & Boro-Sesbania-Fallow & 9180 & 0.11 & 12 & 19 \\
\hline 20 & Boro-Vegetable-T. Aman & 8660 & 0.10 & 9 & 16 \\
\hline 21 & Boro-Aus-Blackgram & 8540 & 0.10 & 5 & 5 \\
\hline 22 & Mustard-Boro-Aus-T. Aman & 7850 & 0.09 & 14 & 30 \\
\hline 23 & Potato-Boro-Aus & 7420 & 0.09 & 4 & 6 \\
\hline 24 & Vegetable-Boro-Fallow & 7100 & 0.08 & 9 & 10 \\
\hline 25 & Boro-Fallow-T. Aman + Fish & 5410 & 0.06 & 3 & 7 \\
\hline
\end{tabular}


Table 4. Continued.

\begin{tabular}{|c|c|c|c|c|c|}
\hline & Cropping pattern & Area (ha) & $\%$ of NCA & No. of district & No. of upazila \\
\hline 26 & Grasspea-Boro-Fallow & 4690 & 0.05 & 8 & 12 \\
\hline 27 & Chilli-Boro-Jute & 3600 & 0.04 & 2 & 3 \\
\hline 28 & Potato-Boro-Aus-T. Aman & 3140 & 0.04 & 5 & 9 \\
\hline 29 & Mustard-Boro-Jute & 3000 & 0.04 & 5 & 7 \\
\hline 30 & Mustard-Boro-Jute-T. Aman & 2980 & 0.03 & 7 & 13 \\
\hline 31 & Grasspea-Boro-B.Aman & 2700 & 0.03 & 3 & 3 \\
\hline 32 & Tobacco-Boro-T. Aman & 2300 & 0.03 & 3 & 4 \\
\hline 33 & Potato-Boro-Jute-T. Aman & 2160 & 0.03 & 4 & 5 \\
\hline 34 & B.gram(Fodder)-Boro-B.Aman & 2000 & 0.02 & 1 & 1 \\
\hline 35 & Grasspea-Boro-Aus & 1800 & 0.02 & 1 & 1 \\
\hline 36 & Boro-Fallow-Maize & 1500 & 0.02 & 1 & 1 \\
\hline 37 & Maize-Boro-T. Aman & 1400 & 0.02 & 2 & 2 \\
\hline 38 & Vegetab-Boro-Aus-T. Aman & 820 & 0.01 & 4 & 5 \\
\hline 39 & Vegetable-Boro-Jute & 800 & 0.01 & 2 & 3 \\
\hline 40 & Mustard-Boro-Sesbania & 700 & 0.01 & 2 & 2 \\
\hline 41 & Potato-Boro-Vegetable & 700 & 0.01 & 4 & 4 \\
\hline 42 & Potato-Boro-Jute & 590 & 0.01 & 2 & 2 \\
\hline 43 & Boro-Maize-Fallow & 410 & 0.00 & 2 & 2 \\
\hline 44 & Boro-Chilli-Fallow & 350 & 0.00 & 2 & 1 \\
\hline \multirow[t]{2}{*}{45} & Boro-Maize-Blackgram & 350 & 0.00 & 1 & 1 \\
\hline & Total Boro area & 4706310 & 54.94 & - & - \\
\hline
\end{tabular}

Table 5. List ofcropping patterns with T. Aman rice and area coverage, 2014-15.

\begin{tabular}{|c|c|c|c|c|c|}
\hline & Cropping pattern & Area (ha) & $\%$ of NCA & No. of district & No. of upazila \\
\hline 01 & Boro-Fallow-T. Aman & 2306005 & 26.919 & 63 & 426 \\
\hline 02 & Fallow-Fallow-T. Aman & 509480 & 5.947 & 36 & 162 \\
\hline 03 & Boro-Aus-T. Aman & 209015 & 2.440 & 47 & 177 \\
\hline 04 & Fallow-Aus-T. Aman & 193275 & 2.256 & 30 & 108 \\
\hline 05 & Mustard-Boro-T. Aman & 184620 & 2.155 & 51 & 203 \\
\hline 06 & Potato-Boro-T. Aman & 180380 & 2.106 & 33 & 115 \\
\hline 07 & Wheat-Jute-T. Aman & 147210 & 1.718 & 43 & 216 \\
\hline 08 & Grasspea-Fallow-T. Aman & 108150 & 1.262 & 25 & 80 \\
\hline 09 & Maize-Fallow-T. Aman & 101460 & 1.184 & 39 & 126 \\
\hline 10 & Wheat-Fallow-T. Aman & 90910 & 1.061 & 39 & 100 \\
\hline 11 & Mungbean-Fallow-T. Aman & 89650 & 1.047 & 22 & 70 \\
\hline 12 & Grasspea-Aus-T. Aman & 81610 & 0.953 & 19 & 61 \\
\hline 13 & Vegetable-Fallow-T. Aman & 74710 & 0.872 & 45 & 170 \\
\hline 14 & Onion-Jute-T. Aman & 54185 & 0.633 & 39 & 102 \\
\hline 15 & Mungbean-Aus-T. Aman & 53730 & 0.627 & 14 & 43 \\
\hline 16 & Chilli-Fallow-T. Aman & 52995 & 0.619 & 45 & 146 \\
\hline 17 & Lentil-Jute-T. Aman & 51875 & 0.606 & 34 & 96 \\
\hline 18 & Vegetable-Vegetable-T. Aman & 51745 & 0.604 & 49 & 127 \\
\hline 19 & Potato-Maize-T. Aman & 47690 & 0.557 & 19 & 68 \\
\hline 20 & Boro-Jute-T. Aman & 40440 & 0.472 & 19 & 56 \\
\hline 21 & Felon-Fallow-T. Aman & 37675 & 0.440 & 14 & 53 \\
\hline 22 & Mustard-Boro-B.Aman & 36520 & 0.426 & 14 & 32 \\
\hline 23 & Wheat-Aus-T. Aman & 36425 & 0.425 & 35 & 93 \\
\hline
\end{tabular}


Table 5. Continued.

\begin{tabular}{|c|c|c|c|c|c|}
\hline & Cropping pattern & Area (ha) & $\%$ of NCA & No. of district & No. of upazila \\
\hline 24 & Mustard-Jute-T. Aman & 32740 & 0.382 & 29 & 64 \\
\hline 25 & Groundnut-Fallow-T. Aman & 29210 & 0.341 & 23 & 60 \\
\hline 26 & Vegetable-Aus-T. Aman & 28700 & 0.335 & 42 & 87 \\
\hline 27 & Water Melon-Fallow-T. Aman & 28340 & 0.331 & 17 & 49 \\
\hline 28 & Potato-Jute-T. Aman & 28310 & 0.330 & 36 & 95 \\
\hline 29 & Potato-B.Aman & 23900 & 0.279 & 9 & 25 \\
\hline 30 & Soybean-Fallow-T. Aman & 23670 & 0.276 & 5 & 10 \\
\hline 31 & Soybean-Aus-T. Aman & 23170 & 0.270 & 5 & 8 \\
\hline 32 & Potato-Fallow-T. Aman & 22715 & 0.265 & 34 & 108 \\
\hline 33 & Potato-Aus-T. Aman & 22405 & 0.262 & 29 & 79 \\
\hline 34 & Wheat-Mungbean-T. Aman & 21760 & 0.254 & 20 & 50 \\
\hline 35 & Maize-Jute-T. Aman & 21325 & 0.249 & 18 & 38 \\
\hline 36 & Vegetable-Boro-T. Aman & 21220 & 0.248 & 22 & 42 \\
\hline 37 & Fish-T. Aman & 20400 & 0.238 & 3 & 8 \\
\hline 38 & Soybean-Jute-T. Aman & 20000 & 0.233 & 4 & 6 \\
\hline 39 & Sweet Potato-Fallow-T. Aman & 16990 & 0.198 & 31 & 103 \\
\hline 40 & Wheat-Maize-T. Aman & 16320 & 0.191 & 9 & 14 \\
\hline 41 & Tobacco-Jute-T. Aman & 15200 & 0.177 & 9 & 19 \\
\hline 42 & Mustard-Fallow-T. Aman & 14870 & 0.174 & 33 & 84 \\
\hline 43 & Groundnut- Aus-T. Aman & 14535 & 0.170 & 10 & 18 \\
\hline 44 & Boro-Sesbania-T. Aman & 14490 & 0.169 & 20 & 33 \\
\hline 45 & Chilli-Aus-T. Aman & 14240 & 0.166 & 21 & 38 \\
\hline 46 & Mustard-Aus-T. Aman & 13790 & 0.161 & 30 & 52 \\
\hline 47 & Fallow-Jute-T. Aman & 13620 & 0.159 & 18 & 32 \\
\hline 48 & Garlic-Jute-T. Aman & 13090 & 0.153 & 38 & 78 \\
\hline 49 & Lentil-Fallow-T. Aman & 12680 & 0.148 & 29 & 68 \\
\hline 50 & Potato-Vegetable-T. Aman & 12620 & 0.147 & 30 & 59 \\
\hline 51 & Lentil-Sesame-T. Aman & 12450 & 0.145 & 14 & 33 \\
\hline 52 & Fallow-Sesame-T. Aman & 11640 & 0.136 & 13 & 20 \\
\hline 53 & Vegetable-Jute-T. Aman & 11305 & 0.132 & 25 & 51 \\
\hline 54 & Lentil-Aus-T. Aman & 11255 & 0.131 & 25 & 46 \\
\hline 55 & Wheat-Sesame-T. Aman & 10650 & 0.124 & 15 & 33 \\
\hline 56 & Water Melon-Aus-T. Aman & 10355 & 0.121 & 11 & 14 \\
\hline 57 & Grasspea-Jute-T. Aman & 10300 & 0.120 & 25 & 43 \\
\hline 58 & Sesame-Fallow-T. Aman & 9265 & 0.108 & 19 & 31 \\
\hline 59 & Felon-Aus-T. Aman & 9245 & 0.108 & 7 & 16 \\
\hline 60 & Boro-Vegetable-T. Aman & 8660 & 0.101 & 9 & 16 \\
\hline 61 & Mustard-Boro-Aus-T. Aman & 7850 & 0.092 & 14 & 30 \\
\hline 62 & Tobacco-Maize-T. Aman & 7470 & 0.087 & 5 & 12 \\
\hline 63 & Chilli-Jute-T. Aman & 7250 & 0.085 & 20 & 35 \\
\hline 64 & Fallow-Vegetable-T. Aman & 7130 & 0.083 & 16 & 29 \\
\hline 65 & Lentil-Mungbean-T. Aman & 6955 & 0.081 & 11 & 22 \\
\hline 66 & Onion-Fallow-T. Aman & 6380 & 0.074 & 32 & 64 \\
\hline 67 & Mustard-Maize-T. Aman & 6310 & 0.074 & 9 & 19 \\
\hline 68 & Tobacco-Aus-T. Aman & 6040 & 0.071 & 5 & 12 \\
\hline 69 & Boro-Fallow-T. Aman +Fish & 5410 & 0.063 & 3 & 7 \\
\hline 70 & Tobacco-Fallow-T. Aman & 5310 & 0.062 & 7 & 15 \\
\hline
\end{tabular}


Table 5. Continued.

\begin{tabular}{|c|c|c|c|c|c|}
\hline & Cropping pattern & Area (ha) & $\%$ of NCA & No. of district & No. of upazila \\
\hline 71 & Wheat-Vegetable-T. Aman & 5205 & 0.061 & 15 & 25 \\
\hline 72 & Maize-Mungbean-T. Aman & 4900 & 0.057 & 1 & 3 \\
\hline 73 & Mustard-Mungbean-T. Aman & 4710 & 0.055 & 12 & 18 \\
\hline 74 & Chickpea-Fallow-T. Aman & 4700 & 0.055 & 12 & 32 \\
\hline 75 & Coriander-Jute-T. Aman & 4625 & 0.054 & 17 & 30 \\
\hline 76 & Vegetable-Maize-T. Aman & 4500 & 0.053 & 9 & 15 \\
\hline 77 & Onion-Aus-T. Aman & 4260 & 0.050 & 17 & 26 \\
\hline 78 & Wheat-Fallow-T. Aman (Orchard) & 3700 & 0.043 & 1 & 2 \\
\hline 79 & Garlic-Fallow-T. Aman & 3680 & 0.043 & 31 & 59 \\
\hline 80 & Mustard-Sesame-T. Aman & 3360 & 0.039 & 12 & 15 \\
\hline 81 & Potato-Boro-Aus-T. Aman & 3140 & 0.037 & 5 & 9 \\
\hline 82 & Onion-Sesame-T. Aman & 3080 & 0.036 & 4 & 6 \\
\hline 83 & Maize-Maize-T. Aman & 3070 & 0.036 & 6 & 8 \\
\hline 84 & Mustard-Boro-Jute-T. Aman & 2980 & 0.035 & 7 & 13 \\
\hline 85 & Maize-Aus-T. Aman & 2970 & 0.035 & 17 & 27 \\
\hline 86 & Lentil-Maize-T. Aman & 2920 & 0.034 & 4 & 8 \\
\hline 87 & Potato-Groundnut-T. Aman & 2720 & 0.032 & 3 & 5 \\
\hline 88 & Lentil-Vegetable-T. Aman & 2660 & 0.031 & 11 & 11 \\
\hline 89 & Vegetable-Groundnut-T. Aman & 2660 & 0.031 & 4 & 5 \\
\hline 90 & Garlic-Mungbean-T. Aman & 2500 & 0.029 & 1 & 2 \\
\hline 91 & Garlic-Aus-T. Aman & 2400 & 0.028 & 13 & 20 \\
\hline 92 & Millet(Kaon)-Fallow-T. Aman & 2390 & 0.028 & 6 & 6 \\
\hline 93 & Musk Melon-Fallow-T. Aman & 2310 & 0.027 & 10 & 23 \\
\hline 94 & Tobacco-Boro-T. Aman & 2300 & 0.027 & 3 & 4 \\
\hline 95 & Fallow-Fallow-T. Aman +Fish & 2200 & 0.026 & 2 & 2 \\
\hline 96 & Coriander-Fallow-T. Aman & 2185 & 0.026 & 31 & 62 \\
\hline 97 & Potato-Boro-Jute-T. Aman & 2160 & 0.025 & 4 & 5 \\
\hline 98 & Sweet Potato-Aus-T. Aman & 2150 & 0.025 & 2 & 2 \\
\hline 99 & Sunflower-Fallow-T. Aman & 2100 & 0.025 & 7 & 23 \\
\hline 100 & Potato-Sesame-T. Aman & 2060 & 0.024 & 15 & 23 \\
\hline 101 & Pea-Fallow-T. Aman & 1950 & 0.023 & 18 & 27 \\
\hline 102 & Blackgram-Jute-T. Aman & 1900 & 0.022 & 11 & 14 \\
\hline 103 & Maize-Sesame-T. Aman & 1835 & 0.021 & 6 & 9 \\
\hline 104 & Maize-Vegetable-T. Aman & 1810 & 0.021 & 11 & 12 \\
\hline 105 & Grasspea-Sesame-T. Aman & 1790 & 0.021 & 5 & 6 \\
\hline 106 & Blackgram-Aus-T. Aman & 1735 & 0.020 & 10 & 13 \\
\hline 107 & Tobacco-Sesbania-T. Aman & 1620 & 0.019 & 1 & 2 \\
\hline 108 & Groundnut-Jute-T. Aman & 1530 & 0.018 & 7 & 13 \\
\hline 109 & Onion-Maize-T. Aman & 1490 & 0.017 & 7 & 9 \\
\hline 110 & Chilli-Vegetable-T. Aman & 1405 & 0.016 & 15 & 19 \\
\hline 111 & Maize-Boro-T. Aman & 1400 & 0.016 & 2 & 2 \\
\hline 112 & Blackcumin-Jute-T. Aman & 1260 & 0.015 & 6 & 11 \\
\hline 113 & Sesame-Aus-T. Aman & 1180 & 0.014 & 4 & 5 \\
\hline 114 & Potato-Mungbean-T. Aman & 1140 & 0.013 & 11 & 13 \\
\hline 115 & Fallow-Fallow-T. Aman (Orchard) & 1000 & 0.012 & 1 & 1 \\
\hline 116 & Coriander-Sesame- T.Aman & 845 & 0.010 & 5 & 5 \\
\hline 117 & Vegetable-Boro-Aus-T.Aman & 820 & 0.010 & 4 & 5 \\
\hline 118 & Potato+Sweet gourd-Aus- T.Aman & 780 & 0.009 & 3 & 5 \\
\hline
\end{tabular}


Table 5. Continued.

\begin{tabular}{lrrrr}
\hline Cropping pattern & Area (ha) & \% of NCA & No. of district & No. of upazila \\
\hline 119 Sweet Potato-Jute- T.Aman & 675 & 0.008 & 10 & 2 \\
120 Potato-Onion- T.Aman & 510 & 0.006 & 5 & 2 \\
121 Grasspea-Mungbean- T.Aman & 440 & 0.005 & 5 & 5 \\
122 Chickpea-Aus-T. Aman & 375 & 0.004 & 2 & 7 \\
123 Sesame-Jute- T.Aman & 290 & 0.003 & 2 & 13 \\
124 Chickpea-Jute-T. Aman & 270 & 0.003 & 0.002 & 1 \\
125 Potato+Maize-Vegetable- T.Aman & 210 & 0.002 & 9 & 1 \\
126 Vegetable-Onion-Jute- T.Aman & 200 & 0.002 & 0.001 & 1 \\
127 Mungbean-Jute- T.Aman & 180 & 0.001 & 1 \\
128 Lentil-Mungbean-Jute- T.Aman & 100 & 0.001 & 1 \\
129 Mustard-Mungbean-Aus- T.Aman & 100 & 0.001 & 1 \\
130 Potato+S. gourd-M.bean- T.Aman & 80 & 65.077 & 1 \\
131 Tobacco-Mungbean- T.Aman & 50 & & 1 \\
\hline
\end{tabular}

Table 6. List ofcropping patterns with B. Aman rice and area coverage, 2014-15.

\begin{tabular}{|c|c|c|c|c|c|}
\hline & Cropping pattern & Area (ha) & $\%$ of NCA & No. of district & No. of upazila \\
\hline 01 & Boro-B. Aman & 183070 & 2.14 & 32 & 113 \\
\hline 02 & Mustard-Boro-B. Aman & 36520 & 0.43 & 14 & 32 \\
\hline 03 & Potato-B. Aman & 23900 & 0.28 & 9 & 25 \\
\hline 04 & Grasspea-B. Aman & 21480 & 0.25 & 21 & 52 \\
\hline 05 & Mustard-B. Aman & 17210 & 0.20 & 12 & 24 \\
\hline 06 & Fallow-B. Aman & 16360 & 0.19 & 15 & 29 \\
\hline 07 & Wheat-B. Aman & 13835 & 0.16 & 16 & 32 \\
\hline 08 & Onion-B. Aman & 10905 & 0.13 & 14 & 27 \\
\hline 09 & Garlic-B. Aman & 10850 & 0.13 & 15 & 21 \\
\hline 10 & Chilli-B. Aman & 9120 & 0.11 & 10 & 23 \\
\hline 11 & Blackgram-B. Aman & 7625 & 0.09 & 11 & 24 \\
\hline 12 & Lentil-B. Aman & 6550 & 0.08 & 16 & 30 \\
\hline 13 & Soybean-B. Aman & 5650 & 0.07 & 4 & 4 \\
\hline 14 & Vegetable-B. Aman & 5200 & 0.06 & 9 & 17 \\
\hline 15 & Maize-B. Aman & 5030 & 0.06 & 10 & 15 \\
\hline 16 & Fallow-B. Aus+B. Aman & 3470 & 0.04 & 3 & 5 \\
\hline 17 & Groundnut-B. Aman & 2820 & 0.03 & 5 & 7 \\
\hline 18 & Fallow-Sesame+B. Aman & 2750 & 0.03 & 5 & 7 \\
\hline 19 & Grasspea-Boro-B. Aman & 2700 & 0.03 & 3 & 3 \\
\hline 20 & Pea-B. Aman & 2620 & 0.03 & 11 & 16 \\
\hline 21 & Onion-Sesame+B. Aman & 2100 & 0.02 & 2 & 3 \\
\hline 22 & B.gram(Fodder)-Boro-B.Aman & 2000 & 0.02 & 1 & 1 \\
\hline 23 & Grasspea-Sesame+B. Aman & 1800 & 0.02 & 2 & 2 \\
\hline 24 & Mustard-B.Aus+B. Aman & 1780 & 0.02 & 4 & 4 \\
\hline 25 & Mustard-Sesame+B. Aman & 1650 & 0.02 & 3 & 4 \\
\hline 26 & Grasspea-B.Aus+B. Aman & 1580 & 0.02 & 6 & 8 \\
\hline 27 & Coriander-B. Aman & 1480 & 0.02 & 11 & 1 \\
\hline 28 & Lentil-Sesame+B. Aman & 980 & 0.01 & 1 & 1 \\
\hline 29 & Sweet Potato-B. Aman & 740 & 0.01 & 6 & 1 \\
\hline 30 & Sesame+B. Aman-Blackgram & 600 & 0.01 & 1 & 3 \\
\hline 31 & Water Melon-B. Aman & 535 & 0.01 & 3 & 2 \\
\hline
\end{tabular}


Table 6. Continued.

\begin{tabular}{|c|c|c|c|c|c|}
\hline & Cropping pattern & Area (ha) & $\%$ of NCA & No. of district & No. of upazila \\
\hline 32 & Potato+Maize-B. Aman & 410 & 0.00 & 2 & 3 \\
\hline 33 & Sesame-B. Aman & 330 & 0.00 & 2 & 2 \\
\hline 34 & Garlic+Muskmelon-B. Aman & 320 & 0.00 & 1 & 2 \\
\hline 35 & Garlic+Water Melon-B. Aman & 320 & 0.00 & 1 & 2 \\
\hline 36 & Musk Melon-B. Aman & 215 & 0.00 & 2 & 5 \\
\hline \multirow[t]{2}{*}{37} & Mungbean-B. Aus+B. Aman & 130 & 0.00 & 4 & 4 \\
\hline & Total of B. Aman & 404635 & 4.72 & - & - \\
\hline
\end{tabular}

Table 7. List of cropping patterns with Aus rice and area coverage, 2014-15.

\begin{tabular}{|c|c|c|c|c|c|}
\hline & Cropping pattern & Area (ha) & $\%$ of NCA & No. of district & No. of upazila \\
\hline 01 & Boro-Aus-T. Aman & 209015 & 2.44 & 47 & 177 \\
\hline 02 & Fallow-Aus-T. Aman & 193275 & 2.26 & 30 & 108 \\
\hline 03 & Grasspea-Aus-T. Aman & 81610 & 0.95 & 19 & 61 \\
\hline 04 & Mungbean-Aus-T. Aman & 53730 & 0.63 & 14 & 43 \\
\hline 05 & Boro-Aus-Fallow & 45865 & 0.54 & 34 & 71 \\
\hline 06 & Wheat-Aus-T. Aman & 36425 & 0.43 & 35 & 93 \\
\hline 07 & Vegetable-Aus-T. Aman & 28700 & 0.34 & 42 & 87 \\
\hline 08 & Soybean-Aus-T. Aman & 23170 & 0.27 & 5 & 8 \\
\hline 09 & Potato-Aus-T. Aman & 22405 & 0.26 & 29 & 79 \\
\hline 10 & Mustard-Boro-Aus & 18140 & 0.21 & 10 & 16 \\
\hline 11 & Wheat-Aus-Fallow & 16200 & 0.19 & 17 & 26 \\
\hline 12 & Vegetable-Aus-Fallow & 15940 & 0.19 & 23 & 41 \\
\hline 13 & Groundnut- Aus-T. Aman & 14535 & 0.17 & 10 & 18 \\
\hline 14 & Chilli-Aus-T. Aman & 14240 & 0.17 & 21 & 38 \\
\hline 15 & Mustard-Aus-T. Aman & 13790 & 0.16 & 30 & 52 \\
\hline 16 & Maize-Aus-Fallow & 13615 & 0.16 & 14 & 18 \\
\hline 17 & Wheat-Aus-Blackgram & 13000 & 0.15 & 6 & 8 \\
\hline 18 & Fallow-Aus+Non-rice (zhum) & 11900 & 0.14 & 3 & 20 \\
\hline 19 & Lentil-Aus-T. Aman & 11255 & 0.13 & 25 & 46 \\
\hline 20 & Water Melon-Aus-T. Aman & 10355 & 0.12 & 11 & 14 \\
\hline 21 & Felon-Aus-T. Aman & 9245 & 0.11 & 7 & 16 \\
\hline 22 & Onion-Aus-Fallow & 8705 & 0.10 & 12 & 17 \\
\hline 23 & Boro-Aus-Blackgram & 8540 & 0.10 & 5 & 5 \\
\hline 24 & Mustard-Boro-Aus-T. Aman & 7850 & 0.09 & 14 & 30 \\
\hline 25 & Chilli-Aus-Fallow & 7565 & 0.09 & 14 & 27 \\
\hline 26 & Potato-Boro-Aus & 7420 & 0.09 & 4 & 6 \\
\hline 27 & Tobacco-Aus-T. Aman & 6040 & 0.07 & 5 & 12 \\
\hline 28 & Mustard-Aus-Fallow & 4935 & 0.06 & 15 & 22 \\
\hline 29 & Lentil-Aus-Fallow & 4570 & 0.05 & 11 & 15 \\
\hline 30 & Potato-Maize-Aus & 4300 & 0.05 & 3 & 4 \\
\hline 31 & Onion-Aus-T. Aman & 4260 & 0.05 & 17 & 26 \\
\hline 32 & Fallow-B. Aus+B. Aman & 3470 & 0.04 & 3 & 5 \\
\hline 33 & Vegetable-Onion-Aus & 3200 & 0.04 & 3 & 3 \\
\hline 34 & Tobacco-Aus-Fallow & 3180 & 0.04 & 5 & 10 \\
\hline 35 & Potato-Boro-Aus-T. Aman & 3140 & 0.04 & 5 & 9 \\
\hline 36 & Maize-Aus-Vegetable & 3000 & 0.04 & 3 & 4 \\
\hline 37 & Maize-Aus-T. Aman & 2970 & 0.03 & 17 & 27 \\
\hline
\end{tabular}


Table 7. Continued.

\begin{tabular}{|c|c|c|c|c|c|}
\hline & Cropping pattern & Area (ha) & $\%$ of NCA & No. of district & No. of upazila \\
\hline 38 & Garlic-Aus-T. Aman & 2400 & 0.03 & 13 & 20 \\
\hline 39 & Potato-Aus-Fallow & 2310 & 0.03 & 13 & 16 \\
\hline 40 & Sweet Potato-Aus-T. Aman & 2150 & 0.03 & 2 & 2 \\
\hline 41 & Mustard-Aus-Blackgram & 1900 & 0.02 & 2 & 2 \\
\hline 42 & Wheat-Aus-Onion & 1900 & 0.02 & 1 & 1 \\
\hline 43 & Onion-Aus-Blackgram & 1810 & 0.02 & 5 & 6 \\
\hline 44 & Grasspea-Boro-Aus & 1800 & 0.02 & 1 & 1 \\
\hline 45 & Mustard-B. Aus+B. Aman & 1780 & 0.02 & 4 & 4 \\
\hline 46 & Blackgram-Aus-T. Aman & 1735 & 0.02 & 10 & 13 \\
\hline 47 & Vegetable-Aus-Blackgram & 1610 & 0.02 & 4 & 4 \\
\hline 48 & Grasspea-B.Aus+B. Aman & 1580 & 0.02 & 6 & 8 \\
\hline 49 & Groundnut- Aus-Fallow & 1550 & 0.02 & 5 & 5 \\
\hline 50 & Garlic-Aus-Fallow & 1450 & 0.02 & 12 & 15 \\
\hline 51 & Fallow-Aus-Fallow & 1400 & 0.02 & 3 & 3 \\
\hline 52 & Maize-Aus-Blackgram & 1370 & 0.02 & 3 & 3 \\
\hline 53 & Cotton-Aus-Fallow & 1330 & 0.02 & 3 & 3 \\
\hline 54 & Pea-Aus-Vegetable & 1310 & 0.02 & 7 & 8 \\
\hline 55 & Sesame-Aus-Fallow & 1190 & 0.01 & 3 & 4 \\
\hline 56 & Sesame-Aus-T. Aman & 1180 & 0.01 & 4 & 5 \\
\hline 57 & Potato-Maize-Aus-Vegetab & 1030 & 0.01 & 3 & 3 \\
\hline 58 & Potato-Sweet gourd-Aus & 910 & 0.01 & 6 & 8 \\
\hline 59 & Vegetable-Boro-Aus-T. Aman & 820 & 0.01 & 4 & 5 \\
\hline 60 & Potato+S.gourd-Aus-T. Aman & 780 & 0.01 & 3 & 5 \\
\hline 61 & Soybean-Aus-Fallow & 700 & 0.01 & 1 & 1 \\
\hline 62 & Wheat-Aus-Cotton & 600 & 0.01 & 1 & 1 \\
\hline 63 & Felon-Aus-Fallow & 470 & 0.01 & 2 & 3 \\
\hline 64 & Chickpea-Aus-T. Aman & 375 & 0.00 & 5 & 7 \\
\hline 65 & Potato-Sesame-Aus & 360 & 0.00 & 4 & 4 \\
\hline 66 & Grasspea-Aus-Blackgram & 250 & 0.00 & 1 & 1 \\
\hline 67 & Mungbean-B. Aus+B. Aman & 130 & 0.00 & 4 & 4 \\
\hline 68 & Must.-M.bean-Aus-T.Aman & 100 & 0.00 & 1 & 1 \\
\hline \multirow[t]{2}{*}{69} & Mungbean-Aus-Fallow & 20 & 0.00 & 1 & 1 \\
\hline & Total of Aus & 977855 & 11.41 & - & - \\
\hline
\end{tabular}

Table 8. List of cropping patterns exclusively with non-rice crops and area coverage, 2014-15.

\begin{tabular}{|c|c|c|c|c|c|}
\hline & Cropping pattern & Area (ha) & $\%$ of NCA & No. of district & No. of upazila \\
\hline 01 & Vegetab-Vegetab-Vegetab & 143270 & 1.672 & 61 & 283 \\
\hline 02 & Vegetable-Vegetable-F & 63935 & 0.746 & 59 & 168 \\
\hline 03 & Wheat-Jute-Fallow & 48700 & 0.568 & 32 & 82 \\
\hline 04 & Onion-Jute-Fallow & 45200 & 0.528 & 29 & 67 \\
\hline 05 & Lentil-Jute-Fallow & 32360 & 0.378 & 27 & 67 \\
\hline 06 & Vegetable-Fallow-Fallow & 31115 & 0.363 & 35 & 84 \\
\hline 07 & Maize-Jute-Fallow & 21425 & 0.250 & 19 & 33 \\
\hline 08 & Grasspea-Jute-Fallow & 21000 & 0.245 & 21 & 39 \\
\hline 09 & Mustard-Jute-Fallow & 17180 & 0.201 & 16 & 35 \\
\hline 10 & Groundnut-Fallow-Fallow & 16485 & 0.192 & 35 & 76 \\
\hline
\end{tabular}


Table 8. Continued.

\begin{tabular}{|c|c|c|c|c|c|}
\hline & Cropping pattern & Area (ha) & $\%$ of NCA & No. of district & No. of upazila \\
\hline 11 & Potato-Jute-Fallow & 14515 & 0.169 & 25 & 53 \\
\hline 12 & Garlic-Jute-Fallow & 13475 & 0.157 & 26 & 48 \\
\hline 13 & Chilli-Jute-Fallow & 13260 & 0.155 & 22 & 54 \\
\hline 14 & Vegetable-Jute-Fallow & 13185 & 0.154 & 26 & 47 \\
\hline 15 & Maize-Fallow-Fallow & 12920 & 0.151 & 22 & 38 \\
\hline 16 & Onion-Vegtable-Vegetable & 11735 & 0.137 & 39 & 87 \\
\hline 17 & Potato-Sesbania & 10770 & 0.126 & 05 & 15 \\
\hline 18 & Potato-Maize-Fallow & 9640 & 0.113 & 10 & 16 \\
\hline 19 & Chilli-Fallow-Fallow & 9265 & 0.108 & 40 & 66 \\
\hline 20 & Chilli-Vegetable-Fallow & 8810 & 0.103 & 44 & 104 \\
\hline 21 & Fallow-Fallow-Blackgram & 8695 & 0.102 & 34 & 59 \\
\hline 22 & Sweet Potato-Fallow-Fallow & 7980 & 0.093 & 37 & 98 \\
\hline 23 & Coriander-Jute-Fallow & 7390 & 0.086 & 15 & 25 \\
\hline 24 & Maize-Vegetable-Fallow & 7120 & 0.083 & 14 & 19 \\
\hline 25 & Lentil-Vegetable-Vegetable & 6330 & 0.074 & 19 & 30 \\
\hline 26 & Wheat-Vegetable-Vegetable & 6100 & 0.071 & 16 & 21 \\
\hline 27 & Garlic-Vegetable-Vegetable & 4385 & 0.051 & 27 & 68 \\
\hline 28 & Tobacco-Jute-Fallow & 4050 & 0.047 & 04 & 8 \\
\hline 29 & Wheat-Jute-Blackgram & 3910 & 0.046 & 07 & 9 \\
\hline 30 & Wheat-Fallow-Fallow & 3810 & 0.044 & 06 & 8 \\
\hline 31 & Wheat-Chilli-Fallow & 3780 & 0.044 & 07 & 9 \\
\hline 32 & Maize-Maize-Fallow & 3720 & 0.043 & 03 & 5 \\
\hline 33 & Lentil-Sesame-Fallow & 3680 & 0.043 & 15 & 20 \\
\hline 34 & Maize-Fallow-Blackgram & 3600 & 0.042 & 04 & 4 \\
\hline 35 & Vegetable-Fallow-Blackgram & 3525 & 0.041 & 16 & 33 \\
\hline 36 & Sweet Potato-Jute-Fallow & 3380 & 0.039 & 13 & 21 \\
\hline 37 & Coriander-Fallow-Fallow & 3175 & 0.037 & 41 & 64 \\
\hline 38 & Wheat-Maize-Fallow & 3070 & 0.036 & 02 & 4 \\
\hline 39 & Sesame-Fallow-Blackgram & 3060 & 0.036 & 06 & 10 \\
\hline 40 & Wheat-Mungbean-Fallow & 2875 & 0.034 & 05 & 7 \\
\hline 41 & Lentil-F-F (Orchard) & 2780 & 0.032 & 03 & 5 \\
\hline 42 & Soybean-Fallow-Fallow & 2710 & 0.032 & 03 & 4 \\
\hline 43 & Blackgram-Jute-Fallow & 2295 & 0.027 & 12 & 16 \\
\hline 44 & Grasspea-Fallow-Fallow & 2295 & 0.027 & 09 & 11 \\
\hline 45 & Potato-Maize-Vegetable & 2190 & 0.026 & 04 & 4 \\
\hline 46 & Vegetable-Maize-Fallow & 2180 & 0.025 & 06 & 7 \\
\hline 47 & Wheat-M.bean-F(Orchard) & 2150 & 0.025 & 02 & 3 \\
\hline 48 & Pea-Jute-Fallow & 2100 & 0.025 & 10 & 15 \\
\hline 49 & Coriander-Vegetable-Fallow & 2090 & 0.024 & 20 & 29 \\
\hline 50 & Potato-Chilli-Fallow & 2070 & 0.024 & 10 & 17 \\
\hline 51 & Wheat-Maize-Vegetable & 2000 & 0.023 & 02 & 2 \\
\hline 52 & Potato-Sesame-Fallow & 1930 & 0.023 & 06 & 10 \\
\hline 53 & Mustard-Jute-Vegetable & 1900 & 0.022 & 03 & 5 \\
\hline 54 & Blackcumin-Jute-Fallow & 1840 & 0.021 & 06 & 12 \\
\hline 55 & Millet(Kaon)+Sesame-Fallow & 1810 & 0.021 & 05 & 7 \\
\hline 56 & Grasspea-Sesbania-Fallow & 1780 & 0.021 & 04 & 4 \\
\hline 57 & Maize-Sesame-Fallow & 1580 & 0.018 & 06 & 8 \\
\hline
\end{tabular}


Table 8. Continued.

\begin{tabular}{|c|c|c|c|c|c|}
\hline & Cropping pattern & Area (ha) & $\%$ of NCA & No. of district & No. of upazila \\
\hline 58 & Wheat-Sesame-Fallow & 1550 & 0.018 & 07 & 8 \\
\hline 59 & Tobacco-Vegetab-Vegetab & 1500 & 0.018 & 01 & 1 \\
\hline 60 & Vegetab-Jute-Vegetable & 1450 & 0.017 & 05 & 5 \\
\hline 61 & Grasspea-Sesame-Fallow & 1330 & 0.016 & 07 & 10 \\
\hline 62 & G.nut-Fallow-Blackgram & 1320 & 0.015 & 03 & 3 \\
\hline 63 & Wheat-Jute-Vegetable & 1320 & 0.015 & 06 & 6 \\
\hline 64 & Onion-Sesame-Fallow & 1270 & 0.015 & 08 & 9 \\
\hline 65 & Water Melon-Fallow-Fallow & 1165 & 0.014 & 09 & 12 \\
\hline 66 & Pea-Vegetable-Fallow & 1160 & 0.014 & 04 & 5 \\
\hline 67 & Potato-Groundnut & 1110 & 0.013 & 05 & 8 \\
\hline 68 & Tobacco-Fallow-Fallow & 1045 & 0.012 & 05 & 8 \\
\hline 69 & Maize-Sesbania-Fallow & 1000 & 0.012 & 01 & 1 \\
\hline 70 & Maize-Mungbean-Vegetable & 950 & 0.011 & 03 & 3 \\
\hline 71 & Millet (Cheena)-F-F & 935 & 0.011 & 05 & 6 \\
\hline 72 & Wheat-Fallow-Blackgram & 900 & 0.011 & 01 & 1 \\
\hline 73 & Mustard-Fallow-Fallow & 895 & 0.010 & 09 & 13 \\
\hline 74 & Wheat-Sesame-B.gram(Orchard) & 760 & 0.009 & 03 & 3 \\
\hline 75 & Maize-Groundnut & 620 & 0.007 & 01 & 1 \\
\hline 76 & Millet(Cheena)-Jute-Fallow & 620 & 0.007 & 02 & 2 \\
\hline 77 & Tobacco-Maize-Vegetable & 600 & 0.007 & 01 & 1 \\
\hline 78 & Tobacco-Sesbania & 600 & 0.007 & 01 & 1 \\
\hline 79 & Groundnut-Sesame-Fallow & 590 & 0.007 & 04 & 5 \\
\hline 80 & Wheat-Ginger/Turmeric & 580 & 0.007 & 02 & 2 \\
\hline 81 & Sweet Potato-Vegetable-Fallow & 540 & 0.006 & 05 & 9 \\
\hline 82 & Garlic-Fallow-Fallow & 530 & 0.006 & 10 & 14 \\
\hline 83 & Onion-Maize-Fallow & 520 & 0.006 & 04 & 4 \\
\hline 84 & Felon-Fallow-Fallow & 380 & 0.004 & 03 & 4 \\
\hline 85 & Groundnut-Millet(Kaon)-F & 380 & 0.004 & 02 & 2 \\
\hline 86 & Mungbean-Jute-Fallow & 300 & 0.004 & 06 & 8 \\
\hline 87 & Mustard-Maize-Jute & 200 & 0.002 & 01 & 1 \\
\hline 88 & Soybean-Jute-Fallow & 140 & 0.002 & 02 & 2 \\
\hline 89 & Sunflower-Jute-Fallow & 135 & 0.002 & 03 & 3 \\
\hline 90 & Muskmelon-Fallow-Fallow & 125 & 0.001 & 05 & 5 \\
\hline 91 & Tobacco-Mungbean-Vegetable & 40 & 0.000 & 01 & 1 \\
\hline \multirow[t]{2}{*}{92} & Barley-Fallow-Fallow & 15 & 0.000 & 01 & 2 \\
\hline & Total of exclusive non-rice area & 714155 & 8.337 & - & - \\
\hline
\end{tabular}

Table 9. List of wheat-based cropping patterns and area coverage, 2014-15.

\begin{tabular}{llrrrr}
\hline & Cropping pattern & Area (ha) & \% of NCA & No. of district & No. of upazila \\
\hline 01 & Wheat-Jute-T. Aman & 147210 & 1.72 & 43 & 216 \\
02 & Wheat-Fallow-T. Aman & 90910 & 1.06 & 39 & 32 \\
03 & Wheat-Jute-Fallow & 48700 & 0.57 & 35 & 82 \\
04 & Wheat-Aus-T. Aman & 36425 & 0.43 & 20 & 93 \\
05 & Wheat-Mungbean-T. Aman & 21760 & 0.25 & 100 & 17 \\
06 & Wheat-Maize-T. Aman & 16320 & 0.19 & 16 & 26 \\
07 & Wheat-Aus-Fallow & 16200 & 0.19 & 30 \\
08 & Wheat-B. Aman & 13835 & 13000 & 0.15 & 6 \\
09 & Wheat-Aus-Blackgram & & & 8 \\
\hline
\end{tabular}


Table 9. Continued.

\begin{tabular}{|c|c|c|c|c|c|}
\hline & Cropping pattern & Area (ha) & $\%$ of NCA & No. of district & No. of upazila \\
\hline 10 & Wheat-Sesame-T. Aman & 10650 & 0.12 & 15 & 33 \\
\hline 11 & Wheat-Vegetable-Vegetable & 6100 & 0.07 & 16 & 21 \\
\hline 12 & Wheat-Vegetable-T. Aman & 5205 & 0.06 & 15 & 25 \\
\hline 13 & Wheat-Jute-Blackgram & 3910 & 0.05 & 7 & 9 \\
\hline 14 & Wheat-Fallow-Fallow & 3810 & 0.04 & 6 & 8 \\
\hline 15 & Wheat-Chilli-Fallow & 3780 & 0.04 & 7 & 9 \\
\hline 16 & Wheat-F-T. Aman(Orchard) & 3700 & 0.04 & 1 & 2 \\
\hline 17 & Wheat-Maize-Fallow & 3070 & 0.04 & 2 & 4 \\
\hline 18 & Wheat-Mungbean-Fallow & 2875 & 0.03 & 5 & 7 \\
\hline 19 & Wheat-Mungbean-F (Orchard) & 2150 & 0.03 & 2 & 3 \\
\hline 20 & Wheat-Maize-Vegetable & 2000 & 0.02 & 2 & 2 \\
\hline 21 & Wheat-Aus-Onion & 1900 & 0.02 & 1 & 1 \\
\hline 22 & Wheat-Sesame-Fallow & 1550 & 0.02 & 7 & 8 \\
\hline 23 & Wheat-Jute-Vegetable & 1320 & 0.02 & 6 & 6 \\
\hline 24 & Wheat-Fallow-Blackgram & 900 & 0.01 & 1 & 1 \\
\hline 25 & Wheat-Sesame-B.gram(Orchard) & 760 & 0.01 & 3 & 3 \\
\hline 26 & Wheat-Aus-Cotton & 600 & 0.01 & 1 & 1 \\
\hline \multirow[t]{2}{*}{27} & Wheat-Ginger/Turmeric & 580 & 0.01 & 2 & 2 \\
\hline & Total of wheat & 459220 & 5.36 & - & - \\
\hline
\end{tabular}

Table 10. List of Maize-based cropping patterns and area coverage, 2014-15.

\begin{tabular}{|c|c|c|c|c|c|}
\hline & Cropping pattern & Area (ha) & $\%$ of NCA & No. of district & No. of upazila \\
\hline 01 & Maize-Fallow-T. Aman & 101460 & 1.18 & 39 & 126 \\
\hline 02 & Potato-Maize-T. Aman & 47690 & 0.56 & 19 & 68 \\
\hline 03 & Maize-Jute-Fallow & 21425 & 0.25 & 19 & 33 \\
\hline 04 & Maize-Jute-T. Aman & 21325 & 0.25 & 18 & 38 \\
\hline 05 & Wheat-Maize-T. Aman & 16320 & 0.19 & 9 & 14 \\
\hline 06 & Maize-Aus-Fallow & 13615 & 0.16 & 14 & 18 \\
\hline 07 & Maize-Fallow-Fallow & 12920 & 0.15 & 22 & 38 \\
\hline 08 & Potato-Maize-Fallow & 9640 & 0.11 & 10 & 16 \\
\hline 09 & Tobacco-Maize-T. Aman & 7470 & 0.09 & 5 & 12 \\
\hline 10 & Maize-Vegetable-Fallow & 7120 & 0.08 & 14 & 19 \\
\hline 11 & Mustard-Maize-T. Aman & 6310 & 0.07 & 9 & 19 \\
\hline 12 & Maize-B. Aman & 5030 & 0.06 & 10 & 15 \\
\hline 13 & Maize-Mungbean-T. Aman & 4900 & 0.06 & 1 & 3 \\
\hline 14 & Vegetable-Maize-T. Aman & 4500 & 0.05 & 9 & 15 \\
\hline 15 & Potato-Maize-Aus & 4300 & 0.05 & 3 & 4 \\
\hline 16 & Maize-Maize-Fallow & 3720 & 0.04 & 3 & 5 \\
\hline 17 & Maize-Fallow-Blackgram & 3600 & 0.04 & 4 & 4 \\
\hline 18 & Maize-Maize-T. Aman & 3070 & 0.04 & 6 & \\
\hline 19 & Wheat-Maize-Fallow & 3070 & 0.04 & 2 & 4 \\
\hline 20 & Maize-Aus-Vegetable & 3000 & 0.04 & 3 & 4 \\
\hline 21 & Maize-Aus-T. Aman & 2970 & 0.03 & 17 & 27 \\
\hline 22 & Lentil-Maize-T. Aman & 2920 & 0.03 & 4 & 8 \\
\hline 23 & Potato-Maize-Vegetable & 2190 & 0.03 & 4 & 4 \\
\hline 24 & Vegetable-Maize-Fallow & 2180 & 0.03 & 6 & 7 \\
\hline 25 & Wheat-Maize-Vegetable & 2000 & 0.02 & 2 & 2 \\
\hline
\end{tabular}


Table 10. Continued.

\begin{tabular}{|c|c|c|c|c|c|}
\hline & Cropping pattern & Area (ha) & $\%$ of NCA & No. of district & No. of upazila \\
\hline 26 & Maize-Sesame-T. Aman & 1835 & 0.02 & 6 & 9 \\
\hline 27 & Maize-Vegetable-T. Aman & 1810 & 0.02 & 11 & 12 \\
\hline 28 & Maize-Sesame-Fallow & 1580 & 0.02 & 6 & 8 \\
\hline 29 & Boro-Fallow-Maize & 1500 & 0.02 & 1 & 1 \\
\hline 30 & Onion-Maize-T. Aman & 1490 & 0.02 & 7 & 9 \\
\hline 31 & Maize-Boro-T. Aman & 1400 & 0.02 & 2 & 2 \\
\hline 32 & Maize-Aus-Blackgram & 1370 & 0.02 & 3 & 3 \\
\hline 33 & Potato-Maize-Aus-Vegetab & 1030 & 0.01 & 3 & 3 \\
\hline 34 & Maize-Sesbania-Fallow & 1000 & 0.01 & 1 & 1 \\
\hline 35 & Maize-Mungbean-Vegetable & 950 & 0.01 & 3 & 3 \\
\hline 36 & Maize-Groundnut & 620 & 0.01 & 1 & 1 \\
\hline 37 & Tobacco-Maize-Vegetable & 600 & 0.01 & 1 & 1 \\
\hline 38 & Onion-Maize-Fallow & 520 & 0.01 & 4 & 4 \\
\hline 39 & Boro-Maize-Fallow & 410 & 0.00 & 2 & 2 \\
\hline 40 & Potato+Maize-B. Aman & 410 & 0.00 & 2 & 3 \\
\hline 41 & Boro-Maize-Blackgram & 350 & 0.00 & 1 & 1 \\
\hline 42 & Potato+Maize-Veg-T.Aman & 210 & 0.00 & 2 & 2 \\
\hline 43 & Mustard-Maize-Jute & 200 & 0.00 & 1 & 1 \\
\hline & Total of maize & 330030 & 3.85 & - & - \\
\hline
\end{tabular}

Table 11. List of cropping patterns with tuber crops and area coverage, 2014-15.

\begin{tabular}{|c|c|c|c|c|c|}
\hline & Cropping pattern & Area (ha) & $\%$ of NCA & No. of district & No. of upazila \\
\hline 01 & Potato-Boro-T. Aman & 180380 & 2.11 & 33 & 115 \\
\hline 02 & Potato-Maize-T. Aman & 47690 & 0.56 & 19 & 68 \\
\hline 03 & Potato-Jute-T. Aman & 28310 & 0.33 & 36 & 95 \\
\hline 04 & Potato-B. Aman & 23900 & 0.28 & 9 & 25 \\
\hline 05 & Potato-Fallow-T. Aman & 22715 & 0.27 & 34 & 108 \\
\hline 06 & Potato-Aus-T. Aman & 22405 & 0.26 & 29 & 79 \\
\hline 07 & Potato-Boro-Fallow & 19360 & 0.23 & 17 & 27 \\
\hline 08 & S.Potato-Fallow-T. Aman & 16990 & 0.20 & 31 & 103 \\
\hline 09 & Potato-Jute-Fallow & 14515 & 0.17 & 25 & 53 \\
\hline 10 & Potato-Vegetable-T. Aman & 12620 & 0.15 & 30 & 59 \\
\hline 11 & Potato-Sesbania & 10770 & 0.13 & 5 & 15 \\
\hline 12 & Potato-Maize-Fallow & 9640 & 0.11 & 10 & 16 \\
\hline 13 & Sweet Potato-Fallow-Fallow & 7980 & 0.09 & 37 & 98 \\
\hline 14 & Potato-Boro-Aus & 7420 & 0.09 & 4 & 6 \\
\hline 15 & Potato-Maize-Aus & 4300 & 0.05 & 3 & 4 \\
\hline 16 & Sweet Potato-Jute-Fallow & 3380 & 0.04 & 13 & 21 \\
\hline 17 & Potato-Boro-Aus-T. Aman & 3140 & 0.04 & 5 & 9 \\
\hline 18 & Potato-Groundnut-T. Aman & 2720 & 0.03 & 3 & 5 \\
\hline 19 & Potato-Aus-Fallow & 2310 & 0.03 & 13 & 16 \\
\hline 20 & Potato-Maize-Vegetable & 2190 & 0.03 & 4 & 4 \\
\hline 21 & Potato-Boro-Jute-T. Aman & 2160 & 0.03 & 4 & 5 \\
\hline 22 & S.Potato-Aus-T. Aman & 2150 & 0.03 & 2 & 2 \\
\hline 23 & Potato-Chilli-Fallow & 2070 & 0.02 & 10 & 17 \\
\hline 24 & Potato-Sesame-T. Aman & 2060 & 0.02 & 15 & 23 \\
\hline 25 & Potato-Sesame-Fallow & 1930 & 0.02 & 6 & 10 \\
\hline
\end{tabular}


Table 11. Continued.

\begin{tabular}{|c|c|c|c|c|c|}
\hline & Cropping pattern & Area (ha) & $\%$ of NCA & No. of district & No. of upazila \\
\hline 26 & Potato-Mungbean-T. Aman & 1140 & 0.01 & 11 & 13 \\
\hline 27 & Potato-Groundnut & 1110 & 0.01 & 5 & 8 \\
\hline 28 & Potato-Maize-Aus-Vegetable & 1030 & 0.01 & 3 & 3 \\
\hline 29 & Potato-Sweet gourd-Aus & 910 & 0.01 & 6 & 8 \\
\hline 30 & Potato+S. gourd-Aus-T. Aman & 780 & 0.01 & 3 & 5 \\
\hline 31 & Sweet Potato-B. Aman & 740 & 0.01 & 6 & 6 \\
\hline 32 & Potato-Boro-Vegetable & 700 & 0.01 & 4 & 4 \\
\hline 33 & Sweet Potato-Jute-T. Aman & 675 & 0.01 & 10 & 13 \\
\hline 34 & Potato-Boro-Jute & 590 & 0.01 & 2 & 2 \\
\hline 35 & Sweet Potato-Vegetable-Fallow & 540 & 0.01 & 5 & 9 \\
\hline 36 & Potato-Onion-T. Aman & 510 & 0.01 & 2 & 2 \\
\hline 37 & Potato+Maize-B. Aman & 410 & 0.00 & 2 & 3 \\
\hline 38 & Potato-Sesame-Aus & 360 & 0.00 & 4 & 4 \\
\hline 39 & Potato+Maize-Vegetab-T. Aman & 210 & 0.00 & 2 & 2 \\
\hline \multirow[t]{2}{*}{40} & Potato+S.gourd-Mung-T Aman & 80 & 0.00 & 1 & 1 \\
\hline & Total of tuber crops & 462890 & 5.40 & - & - \\
\hline
\end{tabular}

not only indicated their dominance, but also their combination diversity among themselves in growing seasons round the year. Because of increasing projected population importance of rice will increase furthermore in future (Kabir et al., 2015). In this table, it was noticeable that only single rice containing CPs occupied large area of the country. The most dominant of them was Boro-Fallow-Fallow followed by FallowFallow-T. Aman. These two CPs occupied $13.3 \%$ and $5.95 \%$ of the NCA, respectively and together occupied $19.25 \%$ of the NCA. BoroFallow-Fallow is practiced in medium lowland to very lowland where inclusion of other crop is difficult. However, some of these areas, DWR can be included after Boro or short duration mustard can be included before Boro. Farmers are reluctant to cultivate DWR because of its low yield. However, short duration mustard can be included in it even with zero tillage. Single T. Aman CP is practiced in constraints soils e.g., in drought prone Barind tract or in tidal wetland. Soil in Barind tract quickly dries up in Rabi season and Rabi crop cultivation is not possible if there is no irrigation facility. In tidal saline wetland, salinity restricts other crops to be included in dry season. In nonsaline tidal wetland, in many areas water recedes late, however inundation by tidal water starts early. So Rabi crop is difficult to grow. Some of these CPs, may possess scope of improvement in suitable areas by including non-rice crops in it and hence increase intensity and diversity. For example, short duration mustard can be included in Boro-Fallow-Fallow CP. Similarly short duration Rabi crops may be included in Fallow-Fallow-T. Aman CP.

Among the rice growing seasons, Boro is the most favourable where irrigation is made ensured. Though the area coverage of Boro stands second, next to T. Aman, it produces the highest amount of rice. Boro rice is cultivated in about 4.7 million hectares of land and Boro containing CPs occupied about $55 \%$ of NCA (Table 4). The highest Boro containing $\mathrm{CP}$ was Boro-Fallow- T. Aman with $26.92 \%$ of NCA. The area under this $\mathrm{CP}$ was about 2.3 million hectares. This CP was followed by Boro-FallowFallow and then Boro-Aus- T. Aman with 13.3\% and $2.44 \%$ of NCA. There were also some minor CPs with Boro e.g., Blackgram (Fodder)-Boro-B. Aman, Grasspea-Boro-Aus etc.

In traditional agriculture, Aman was considered as the most suited season for rice culture in Bangladesh. Before the development of modern irrigation system it was only the major season for rice culture. Rice requires enough water for successful culture. Monsoon rain provides Aman rice with enough water. However, unlike Boro, it is practically rainfed 
and rainfall in its growing period is unreliable. During this time other sources of water like flood, tidal water etc may be available and are adjusted to its culture. Because of different growing environments of Aman rice there are different types of Aman of which transplanted and broadcasted are the common types. Because of these varied growing environments, Aman grows in many CPs. Table 5 presents T. Aman containing CPs. There were $131 \mathrm{CPs}$ containing T. Aman in it. Boro-Fallow-T. Aman was the most dominant $\mathrm{CP}$ accounting for about $26.92 \%$ of the NCA. The following CPs were FallowFallow-T. Aman, Boro-Aus-T. Aman, FallowAus-T. Aman accounting for about 5.95\%, $2.44 \%$ and $2.26 \%$ of the NCA, respectively. There were some minor CPs in terms of area coverage containing T. Aman in them. Such as Lentil-Mungbean-Jute-T. Aman, MustardMungbean-Aus-T. Aman, Potato Sweet gourdMungbean-T. Aman, Tobacco-Mungbean-T. Aman etc and each of which accounts for about $0.001 \%$ of NCA, separately.

Broadcast Aman is suited well in flood prone areas. Table 6 presents CPs with B. Aman. There were $37 \mathrm{CPs}$ containing B. Aman. The most dominant $\mathrm{CP}$ was Boro-B. Aman covering about $2.14 \%$ of NCA. This was followed by Mustard-Boro-B. Aman, PotatoFallow-B. Aman etc. Some B. Aman rice is cultivated as mixed crop with B. Aus or sesame where after harvest of Aus or sesame puddling is not possible and hence T. Aman cultivation is not possible. So, broadcast Aus and Aman are sown together. In some B. Aman-based CPs scavenging fish cultivation may be included in some pocket area in future to utilize the flood water.

Currently, Aus is basically cultured as transplanted crop. In the table, Aus means T. Aus and in case of broadcast Aus it is denoted by B. Aus. There were $69 \mathrm{CPs}$ containing Aus in it (Table 7). These CPs have the coverage of $11.41 \%$ of NCA. The most dominant CP containing Aus in it was Boro-Aus-T. Aman which covered $2.44 \%$ of NCA. This CP was followed by Fallow-Aus- T. Aman and then Grasspea-Aus- T. Aman.
Rice and other cereal containing CPs occupied huge areas of the country (Tables 2 and 3). Contrarily there were other CPs they excluded rice in it. Ninety-two CPs were found excluding rice (Table 8 ). These CPs were minor considering their area coverage and altogether they contributed to $8.34 \%$ of NCA. Many nonrice crops are cultivated in our country. Thus their combination in CPs gave rise to a big number of list of CPs. Because of some special features, these CPs were developed without rice in it. As for example, in the vicinity of a city, vegetable are more profitable, so farmer grows vegetable round the year. Similarly in some charlands only groundnut is cultivated successfully and gives rise to a Groundnut-Fallow-Fallow CP.

There were 27 wheat-based CPs which occupying $5.36 \%$ of NCA (Table 9). WheatJute-T. Aman was the most dominant CP followed by Wheat-Fallow-T. Aman with a NCA of $1.72 \%$ and $1.06 \%$, respectively.

In Bangladesh, maize is cultivated in Rabi and Kharif-I seasons. In recent years, area and production of maize increased considerably. Table 10 presents maize-based CPs. The most dominant CP with maize was Maize-Fallow-T. Aman, which occupied $1.18 \%$ of NCA. Maizebased CPs altogether covered $3.85 \%$ of NCA.

Potato and sweet potato are major tuber crops. These tuber crops occupied $5.4 \%$ of the NCA (Table 11). There were 40 CPs containing potato or sweet potato. Potato-Boro-T. Aman was the most dominant $\mathrm{CP}$ which was followed by Potato-Maize-T. Aman CP. These CPs occupied $2.11 \%$ and $0.56 \%$ of the NCA, respectively.

In Bangladesh there are several edible oil seed crops. Mustard is the major one. Others are very minor. Mustard containing CPs are listed in Table 12. There was 24 mustard containing CPs. All these 24 CPs occupied 520,470 hectares of land accounting for over $6 \%$ of the NCA. Among those, the most dominant $\mathrm{CP}$ was Mustard-Boro-T. Aman and it was followed by Mustard-Boro-Fallow which occupied 184,620 and 143,130 hectares of land and found in 203 and 112 upazilas, respectively. 
Table 12. List of mustard-based cropping patterns and area coverage, 2014-15.

\begin{tabular}{rlrrrr}
\hline & Cropping pattern & Area (ha) & \% of NCA & No. of district & No. of upazila \\
\hline 01 & Mustard-Boro-T. Aman & 184620 & 2.16 & 51 & 203 \\
02 & Mustard-Boro-Fallow & 143130 & 1.67 & 37 & 112 \\
03 & Mustard-Boro-B. Aman & 36520 & 0.43 & 14 & 32 \\
04 & Mustard-Jute-T. Aman & 32740 & 0.38 & 29 & 64 \\
05 & Mustard-Boro-Aus & 18140 & 0.21 & 10 & 16 \\
06 & Mustard-B. Aman & 17210 & 0.20 & 12 & 24 \\
07 & Mustard-Jute-Fallow & 17180 & 0.20 & 16 & 35 \\
08 & Mustard-Fallow-T. Aman & 14870 & 0.17 & 33 & 84 \\
09 & Mustard-Aus-T. Aman & 13790 & 0.16 & 30 & 52 \\
10 & Mustard-Boro-Aus-T. Aman & 7850 & 0.09 & 14 & 30 \\
11 & Mustard-Maize-T. Aman & 6310 & 0.07 & 9 & 19 \\
12 & Mustard-Aus-Fallow & 4935 & 0.06 & 15 & 22 \\
13 & Mustard-M.bean-T. Aman & 4710 & 0.05 & 12 & 18 \\
14 & Mustard-Sesame-T. Aman & 3360 & 0.04 & 12 & 15 \\
15 & Mustard-Boro-Jute & 3000 & 0.04 & 5 & 7 \\
16 & Mustard-Boro-Jute-T. Aman & 2980 & 0.03 & 7 & 13 \\
17 & Mustard-Aus-Blackgram & 1900 & 0.02 & 2 & 2 \\
18 & Mustard-Jute-Vegetable & 1900 & 0.02 & 3 & 5 \\
19 & Mustard-B. Aus+B. Aman & 1780 & 0.02 & 4 & 4 \\
20 & Mustard-Sesame+B. Aman & 1650 & 0.02 & 3 & 4 \\
21 & Mustard-Fallow-Fallow & 895 & 0.01 & 9 & 2 \\
22 & Mustard-Boro-Sesbania & 700 & 0.01 & 2 & 1 \\
23 & Mustard-Maize-Jute & 200 & 0.00 & 1 & - \\
24 & Mustard-M.bean-Aus-T.Aman & 100 & 0.00 & 6.08 & \\
\hline & Total mustard & 520470 & & & 2 \\
\hline
\end{tabular}

They occupied $2.16 \%$ and $1.67 \%$ of the NCA. Mustard containing other CPs neither occupied large area nor showed their presence in greater number of upazilas. Mustard accompanied in these CPs with different rice, maize, jute, mungbean, sesame etc.

There are other edible oil seed crops, which are minor in terms of area coverage. These are sesame, groundnut, soybean, sunflower etc. There were $52 \mathrm{CPs}$ containing minor edible oil seed crops (Table 13). The most dominant $\mathrm{CP}$ was GroundnutFallow-T. Aman followed by SoybeanFallow-T. Aman. These CPs occupied only $0.341 \%$ and $0.276 \%$ of the NCA, respectively. The CP, Groundnut-Fallow-Fallow had low coverage but were present comparatively higher number of 76 upazilas. All these CPs, though occupied few areas $(2.81 \%$ of NCA), perhaps were well fitted to suitable ecosystems in pocket areas.
Grasspea, mungbean, lentil, blackgram, field pea, felon are common pulses of Bangladesh. Most of the pulses are grown in Rabi season. Mungbean is grown in Kharif-I and blackgram is in Kharif-II seasons. There were $83 \mathrm{CPs}$ containing pulses (Table 14). Pulses accompanied in these CPs with different rices, cereals, jute, oil crops, tuber crops. All these $83 \mathrm{CPs}$ together occupied $8.78 \%$ of the NCA. Among those, the most dominant CP was Grasspea-Fallow-T. Aman. This CP was followed by Mungbean-Fallow- T. Aman. The corresponding coverages of these two patterns were 108,150 and 89,650 hectares found in 80 and 70 upazilas, respectively. These CPs occupied $1.26 \%$ and $1.06 \%$ of the NCA. Pulse containing other CPs neither occupied large area nor were present in large number of upazilas. Pulses containing CPs altogether covered $8.78 \%$ of the NCA. 
In Bangladesh there are several fibre crops. Jute is the major one. It grows in Kharif-I season. There were 56 jute containing CPs (Table 15). These 56 CPs occupied $9.09 \%$ of the NCA. Among those, the most dominant CP was Wheat-Jute-T. Aman and it was followed by Onion-Jute-T. Aman which occupied 147,210 and 54,185 hectares of land and were found in 216 and 102 upazilas, respectively. These CPs occupied separately $1.72 \%$ and $0.63 \%$ of the NCA. Jute containing other CPs neither occupied large area nor were present in large number of upazilas. Jute accompanied in these CPs with different rices, mungbean, sesame etc. Jute containing CPs usually contained Rabi or Boro crops before jute and T. Aman and fallow after jute.

$\mathrm{CP}$ with tobacco was cultivated in about 49,000 hectares of land (Table 16). There were 14 tobacco containing CPs and all of these together occupied $0.572 \%$ of NCA. The most dominant $\mathrm{CP}$ with tobacco was TobaccoJute-T. Aman which was followed by TobaccoMaize-T. Aman. Tobacco was cultivated only in 12 districts in three to four clusters viz, Kushtia, Jhenaidaha, Meherpur, Lalmonirhat, Rangpur, Nilphamari, Rangamati, Bandarban, Khagrachhari, Coxsbazaar and Manikganj.

Table 13. List of cropping pattern with minor oil crops and area coverage, 2014-15.

\begin{tabular}{|c|c|c|c|c|c|}
\hline & Cropping pattern & Area (ha) & $\%$ of NCA & No. of district & No. of upazila \\
\hline 1 & Groundnut-Fallow-T. Aman & 29210 & 0.341 & 23 & 60 \\
\hline 2 & Soybean-Fallow-T. Aman & 23670 & 0.276 & 5 & 10 \\
\hline 3 & Soybean-Aus-T. Aman & 23170 & 0.270 & 5 & 8 \\
\hline 4 & Soybean-Jute-T. Aman & 20000 & 0.233 & 4 & 6 \\
\hline 5 & Groundnut-Fallow-Fallow & 16485 & 0.192 & 35 & 76 \\
\hline 6 & Groundnut- Aus-T. Aman & 14535 & 0.170 & 10 & 18 \\
\hline 7 & Lentil-Sesame-T. Aman & 12450 & 0.145 & 14 & 33 \\
\hline 8 & Fallow-Sesame-T. Aman & 11640 & 0.136 & 13 & 20 \\
\hline 9 & Wheat-Sesame-T. Aman & 10650 & 0.124 & 15 & 33 \\
\hline 10 & Sesame-Fallow-T. Aman & 9265 & 0.108 & 19 & 31 \\
\hline 11 & Soybean-B. Aman & 5650 & 0.066 & 4 & 4 \\
\hline 12 & Lentil-Sesame-Fallow & 3680 & 0.043 & 15 & 20 \\
\hline 13 & Mustard-Sesame-T. Aman & 3360 & 0.039 & 12 & 15 \\
\hline 14 & Onion-Sesame-T. Aman & 3080 & 0.036 & 4 & 6 \\
\hline 15 & Sesame-Fallow-Blackgram & 3060 & 0.036 & 6 & 10 \\
\hline 16 & Groundnut-B. Aman & 2820 & 0.033 & 5 & 7 \\
\hline 17 & Fallow-Sesame+B. Aman & 2750 & 0.032 & 5 & 7 \\
\hline 18 & Potato-Groundnut-T. Aman & 2720 & 0.032 & 3 & 5 \\
\hline 19 & Soybean-Fallow-Fallow & 2710 & 0.032 & 3 & 4 \\
\hline 20 & Vegetable-Groundnut-T. Aman & 2660 & 0.031 & 4 & 5 \\
\hline 21 & Onion-Sesame+B. Aman & 2100 & 0.025 & 2 & 3 \\
\hline 22 & Sunflower-Fallow-T. Aman & 2100 & 0.025 & 7 & 23 \\
\hline 23 & Potato-Sesame-T. Aman & 2060 & 0.024 & 15 & 23 \\
\hline 24 & Potato-Sesame-Fallow & 1930 & 0.023 & 6 & 10 \\
\hline 25 & Maize-Sesame-T. Aman & 1835 & 0.021 & 6 & 9 \\
\hline 26 & Millet(Kaon)+Sesame-Fallow & 1810 & 0.021 & 5 & 7 \\
\hline 27 & Grasspea-Sesame+B. Aman & 1800 & 0.021 & 2 & 2 \\
\hline 28 & Grasspea-Sesame-T. Aman & 1790 & 0.021 & 5 & 6 \\
\hline 29 & Mustard-Sesame+B. Aman & 1650 & 0.019 & 3 & 4 \\
\hline 30 & Maize-Sesame-Fallow & 1580 & 0.018 & 6 & 8 \\
\hline
\end{tabular}


Table 13. Continued.

\begin{tabular}{|c|c|c|c|c|c|}
\hline & Cropping pattern & Area (ha) & $\%$ of NCA & No. of district & No. of upazila \\
\hline 31 & Wheat-Sesame-Fallow & 1550 & 0.018 & 7 & 8 \\
\hline 32 & Groundnut- Aus-Fallow & 1550 & 0.018 & 5 & 5 \\
\hline 33 & Groundnut-Jute-T.Aman & 1530 & 0.018 & 7 & 13 \\
\hline 34 & Grasspea-Sesame-Fallow & 1330 & 0.016 & 7 & 10 \\
\hline 35 & Groundnut-Fallow-Blackgram & 1320 & 0.015 & 3 & 3 \\
\hline 36 & Onion-Sesame-Fallow & 1270 & 0.015 & 8 & 9 \\
\hline 37 & Sesame-Aus-Fallow & 1190 & 0.014 & 3 & 4 \\
\hline 38 & Sesame-Aus-T. Aman & 1180 & 0.014 & 4 & 5 \\
\hline 39 & Potato-Groundnut & 1110 & 0.013 & 5 & 8 \\
\hline 40 & Lentil-Sesame+B. Aman & 980 & 0.011 & 1 & 1 \\
\hline 41 & Coriander-Sesame-T. Aman & 845 & 0.010 & 5 & 5 \\
\hline 42 & Wheat-Sesame-Blackgram (Orchard) & 760 & 0.009 & 3 & 3 \\
\hline 43 & Soybean-Aus-Fallow & 700 & 0.008 & 1 & 1 \\
\hline 44 & Maize-Groundnut & 620 & 0.007 & 1 & 1 \\
\hline 45 & Sesame+B.Aman-Blackgram & 600 & 0.007 & 1 & 1 \\
\hline 46 & Groundnut-Sesame-Fallow & 590 & 0.007 & 4 & 5 \\
\hline 47 & Groundnut-Millet (kaon)-Fallow & 380 & 0.004 & 2 & 2 \\
\hline 48 & Potato-Sesame-Aus & 360 & 0.004 & 4 & 4 \\
\hline 49 & Sesame-B. Aman & 330 & 0.004 & 2 & 2 \\
\hline 50 & Sesame-Jute-T. Aman & 290 & 0.003 & 2 & 2 \\
\hline 51 & Soybean-Jute-Fallow & 140 & 0.002 & 2 & 2 \\
\hline 52 & Sunflower-Jute-Fallow & 135 & 0.002 & 3 & 3 \\
\hline & Total for minor edible oil crops & 240980 & 2.813 & - & - \\
\hline
\end{tabular}

Table 14. List of cropping patterns with pulse crops and area coverage, 2014-15.

\begin{tabular}{rlrrrr}
\hline & Cropping pattern & Area (ha) & \% of NCA & No. of district & No. of upazila \\
\hline 1 & Grasspea-Fallow-T. Aman & 108150 & 1.26 & 25 & 80 \\
2 & Mungbean-Fallow-T. Aman & 89650 & 1.05 & 22 & 70 \\
3 & Grasspea-Aus-T. Aman & 81610 & 0.95 & 19 & 61 \\
4 & Mungbean-Aus-T. Aman & 53730 & 0.63 & 14 & 43 \\
5 & Lentil-Jute-T. Aman & 51875 & 0.61 & 34 & 96 \\
6 & Felon-Fallow-T. Aman & 37675 & 0.44 & 14 & 53 \\
7 & Lentil-Jute-Fallow & 32360 & 0.38 & 27 & 67 \\
8 & Wheat-Mungbean-T. Aman & 21760 & 0.25 & 20 & 50 \\
9 & Grasspea-B. Aman & 21480 & 0.25 & 21 & 52 \\
10 & Grasspea-Jute-Fallow & 21000 & 0.25 & 21 & 39 \\
11 & Boro-Fallow-Blackgram & 13270 & 0.15 & 27 & 44 \\
12 & Wheat-Aus-Blackgram & 13000 & 0.15 & 6 & 8 \\
13 & Lentil-Fallow-T. Aman & 12680 & 0.15 & 29 & 68 \\
14 & Lentil-Sesame-T. Aman & 12450 & 0.15 & 14 & 33 \\
15 & Lentil-Aus-T. Aman & 11255 & 0.13 & 25 & 46 \\
16 & Grasspea-Jute-T. Aman & 10300 & 0.12 & 25 & 43 \\
17 & Felon-Aus-T. Aman & 9245 & 0.11 & 7 & 16 \\
18 & Fallow-Fallow-Blackgram & 8695 & 0.10 & 34 & 59 \\
19 & Boro-Aus-Blackgram & 8540 & 0.10 & 5 & 5 \\
20 & Blackgram-B. Aman & 7625 & 0.09 & 11 & 24 \\
\hline
\end{tabular}


Table 14. Continued.

\begin{tabular}{|c|c|c|c|c|c|}
\hline & Cropping pattern & Area (ha) & $\%$ of NCA & No. of district & No. of upazila \\
\hline 21 & Lentil-Mungbean-T. Aman & 6955 & 0.08 & 11 & 22 \\
\hline 22 & Lentil-B. Aman & 6550 & 0.08 & 16 & 30 \\
\hline 23 & Lentil-Vegetable-Vegetable & 6330 & 0.07 & 19 & 30 \\
\hline 24 & Maize-Mungbean-T. Aman & 4900 & 0.06 & 1 & 3 \\
\hline 25 & Mustard-Mungbean-T. Aman & 4710 & 0.05 & 12 & 18 \\
\hline 26 & Chickpea-Fallow-T. Aman & 4700 & 0.05 & 12 & 32 \\
\hline 27 & Grasspea-Boro-Fallow & 4690 & 0.05 & 8 & 12 \\
\hline 28 & Lentil-Aus-Fallow & 4570 & 0.05 & 11 & 15 \\
\hline 29 & Wheat-Jute-Blackgram & 3910 & 0.05 & 7 & 9 \\
\hline 30 & Lentil-Sesame-Fallow & 3680 & 0.04 & 15 & 20 \\
\hline 31 & Maize-Fallow-Blackgram & 3600 & 0.04 & 4 & 4 \\
\hline 32 & Vegetable-Fallow-Blackgram & 3525 & 0.04 & 16 & 33 \\
\hline 33 & Sesame-Fallow-Blackgram & 3060 & 0.04 & 6 & 10 \\
\hline 34 & Lentil-Maize-T. Aman & 2920 & 0.03 & 4 & 8 \\
\hline 35 & Wheat-Mungbean-Fallow & 2875 & 0.03 & 5 & 7 \\
\hline 36 & Lentil-Fallow-Fallow (Orchard) & 2780 & 0.03 & 3 & 5 \\
\hline 37 & Grasspea-Boro-B. Aman & 2700 & 0.03 & 3 & 3 \\
\hline 38 & Lentil-Vegetable-T. Aman & 2660 & 0.03 & 11 & 11 \\
\hline 39 & Fieldpea-B. Aman & 2620 & 0.03 & 11 & 16 \\
\hline 40 & Garlic-Mungbean-T. Aman & 2500 & 0.03 & 1 & 2 \\
\hline 41 & Blackgram-Jute-Fallow & 2295 & 0.03 & 12 & 16 \\
\hline 42 & Grasspea-Fallow-Fallow & 2295 & 0.03 & 9 & 11 \\
\hline 43 & Wheat-Mung-Fallow (Orchard) & 2150 & 0.03 & 2 & 3 \\
\hline 44 & Fieldpea-Jute-Fallow & 2100 & 0.02 & 10 & 15 \\
\hline 45 & Blackgram(Fodder)-Boro-B. Aman & 2000 & 0.02 & 1 & 1 \\
\hline 46 & Fieldpea-Fallow-T. Aman & 1950 & 0.02 & 18 & 27 \\
\hline 47 & Blackgram-Jute-T. Aman & 1900 & 0.02 & 11 & 14 \\
\hline 48 & Mustard-Aus-Blackgram & 1900 & 0.02 & 2 & 2 \\
\hline 49 & Onion-Aus-Blackgram & 1810 & 0.02 & 5 & 6 \\
\hline 50 & Grasspea-Boro-Aus & 1800 & 0.02 & 1 & 1 \\
\hline 51 & Grasspea-Sesame+B. Aman & 1800 & 0.02 & 2 & 2 \\
\hline 52 & Grasspea-Sesame-T. Aman & 1790 & 0.02 & 5 & 6 \\
\hline 53 & Grasspea-Sesbania-Fallow & 1780 & 0.02 & 4 & 4 \\
\hline 54 & Blackgram-Aus-T. Aman & 1735 & 0.02 & 10 & 13 \\
\hline 55 & Vegetab-Aus-Blackgram & 1610 & 0.02 & 4 & 4 \\
\hline 56 & Grasspea-B. Aus+B. Aman & 1580 & 0.02 & 6 & 8 \\
\hline 57 & Maize-Aus-Blackgram & 1370 & 0.02 & 3 & 3 \\
\hline 58 & Grasspea-Sesame-Fallow & 1330 & 0.02 & 7 & 10 \\
\hline 59 & Groundnut-Fallow-Blackgram & 1320 & 0.02 & 3 & 3 \\
\hline 60 & Fieldpea-Aus-Vegetable & 1310 & 0.02 & 7 & 8 \\
\hline 61 & Fieldpea-Vegetable-Fallow & 1160 & 0.01 & 4 & 5 \\
\hline 62 & Potato-Mungbean-T. Aman & 1140 & 0.01 & 11 & 13 \\
\hline 63 & Lentil-Sesame+B. Aman & 980 & 0.01 & 1 & 1 \\
\hline 64 & Maize-Mungbean-Vegetable & 950 & 0.01 & 3 & 3 \\
\hline 65 & Wheat-Fallow-Blackgram & 900 & 0.01 & 1 & 1 \\
\hline 66 & Wheat-Sesame-B.gram (Orchard) & 760 & 0.01 & 3 & 3 \\
\hline 67 & Sesame+B. Aman-Blackgram & 600 & 0.01 & 1 & 1 \\
\hline
\end{tabular}


Table 14. Continued.

\begin{tabular}{|c|c|c|c|c|c|}
\hline & Cropping pattern & Area (ha) & $\%$ of NCA & No. of district & No. of upazila \\
\hline 68 & Felon-Aus-Fallow & 470 & 0.01 & 2 & 3 \\
\hline 69 & Grasspea-Mungbean-T. Aman & 440 & 0.01 & 5 & 5 \\
\hline 70 & Felon-Fallow-Fallow & 380 & 0.00 & 3 & 4 \\
\hline 71 & Chickpea-Aus-T. Aman & 375 & 0.00 & 5 & 7 \\
\hline 72 & Boro-Maize-Blackgram & 350 & 0.00 & 1 & 8 \\
\hline 73 & Mungbean-Jute-Fallow & 300 & 0.00 & 6 & 8 \\
\hline 74 & Chickpea-Jute-T. Aman & 270 & 0.00 & 7 & 11 \\
\hline 75 & Grasspea-Aus-Blackgram & 250 & 0.00 & 1 & 1 \\
\hline 76 & Mungbean-Jute-T. Aman & 180 & 0.00 & 9 & 11 \\
\hline 77 & Mungbean-B. Aus+B. Aman & 130 & 0.00 & 4 & 4 \\
\hline 78 & Lentil-Mungbean-Jute-T. Aman & 100 & 0.00 & 1 & 1 \\
\hline 79 & Mustard-Mung-Aus-T. Aman & 100 & 0.00 & 1 & 1 \\
\hline 80 & Potato + S. gourd-Mung-T Aman & 80 & 0.00 & 1 & 1 \\
\hline 81 & Tobacco-Mungbean-T. Aman & 50 & 0.00 & 1 & 1 \\
\hline 82 & Tobacco-Mungbean-Vegetable & 40 & 0.00 & 1 & 1 \\
\hline \multirow[t]{2}{*}{83} & Mungbean-Aus-Fallow & 20 & 0.00 & 1 & 1 \\
\hline & Total of pulses & 752435 & 8.78 & - & - \\
\hline
\end{tabular}

Table 15. List of jute-based cropping patterns and area coverage, 2014-15.

\begin{tabular}{|c|c|c|c|c|c|}
\hline & Cropping pattern & Area (ha) & $\%$ of NCA & No. of district & No. of upazila \\
\hline 01 & Wheat-Jute-T. Aman & 147210 & 1.72 & 43 & 216 \\
\hline 02 & Onion-Jute-T. Aman & 54185 & 0.63 & 39 & 102 \\
\hline 03 & Lentil-Jute-T. Aman & 51875 & 0.61 & 34 & 96 \\
\hline 04 & Wheat-Jute-Fallow & 48700 & 0.57 & 32 & 82 \\
\hline 05 & Onion-Jute-Fallow & 45200 & 0.53 & 29 & 67 \\
\hline 06 & Boro-Jute-T.Aman & 40440 & 0.47 & 19 & 56 \\
\hline 07 & Mustard-Jute-T. Aman & 32740 & 0.38 & 29 & 64 \\
\hline 08 & Lentil-Jute-Fallow & 32360 & 0.38 & 27 & 67 \\
\hline 09 & Potato-Jute-T. Aman & 28310 & 0.33 & 36 & 95 \\
\hline 10 & Maize-Jute-Fallow & 21425 & 0.25 & 19 & 33 \\
\hline 11 & Maize-Jute-T. Aman & 21325 & 0.25 & 18 & 38 \\
\hline 12 & Grasspea-Jute-Fallow & 21000 & 0.25 & 21 & 39 \\
\hline 13 & Soybean-Jute-T. Aman & 20000 & 0.23 & 4 & 6 \\
\hline 14 & Mustard-Jute-Fallow & 17180 & 0.20 & 16 & 35 \\
\hline 15 & Boro-Jute-Fallow & 16370 & 0.19 & 19 & 25 \\
\hline 16 & Tobacco-Jute-T. Aman & 15200 & 0.18 & 9 & 19 \\
\hline 17 & Potato-Jute-Fallow & 14515 & 0.17 & 25 & 53 \\
\hline 18 & Fallow-Jute-T. Aman & 13620 & 0.16 & 18 & 32 \\
\hline 19 & Garlic-Jute-Fallow & 13475 & 0.16 & 26 & 48 \\
\hline 20 & Chilli-Jute-Fallow & 13260 & 0.15 & 22 & 54 \\
\hline 21 & Vegetable-Jute-Fallow & 13185 & 0.15 & 26 & 47 \\
\hline 22 & Garlic-Jute-T. Aman & 13090 & 0.15 & 38 & 78 \\
\hline 23 & Vegetable-Jute-T. Aman & 11305 & 0.13 & 25 & 51 \\
\hline 24 & Grasspea-Jute-T. Aman & 10300 & 0.12 & 25 & 43 \\
\hline 25 & Coriander-Jute-Fallow & 7390 & 0.09 & 15 & 25 \\
\hline 26 & Chilli-Jute-T. Aman & 7250 & 0.08 & 20 & 35 \\
\hline 27 & Coriander-Jute-T. Aman & 4625 & 0.05 & 17 & 30 \\
\hline
\end{tabular}


Table 15. Continued.

\begin{tabular}{|c|c|c|c|c|c|}
\hline & Cropping pattern & Area (ha) & $\%$ of NCA & No. of district & No. of upazila \\
\hline 28 & Tobacco-Jute-Fallow & 4050 & 0.05 & 4 & 8 \\
\hline 29 & Wheat-Jute-Blackgram & 3910 & 0.05 & 7 & 9 \\
\hline 30 & Chilli-Boro-Jute & 3600 & 0.04 & 2 & 3 \\
\hline 31 & Sweet Potato-Jute-Fallow & 3380 & 0.04 & 13 & 21 \\
\hline 32 & Mustard-Boro-Jute & 3000 & 0.04 & 5 & 7 \\
\hline 33 & Mustard-Boro-Jute-T. Aman & 2980 & 0.03 & 7 & 13 \\
\hline 34 & Blackgram-Jute-Fallow & 2295 & 0.03 & 12 & 16 \\
\hline 35 & Potato-Boro-Jute-T. Aman & 2160 & 0.03 & 4 & 5 \\
\hline 36 & Fieldpea-Jute-Fallow & 2100 & 0.02 & 10 & 15 \\
\hline 37 & Blackgram-Jute-T. Aman & 1900 & 0.02 & 11 & 14 \\
\hline 38 & Mustard-Jute-Vegetable & 1900 & 0.02 & 3 & 5 \\
\hline 39 & Blackcumin-Jute-Fallow & 1840 & 0.02 & 6 & 12 \\
\hline 40 & Groundnut-Jute-T. Aman & 1530 & 0.02 & 7 & 13 \\
\hline 41 & Vegetable-Jute-Vegetable & 1450 & 0.02 & 5 & 5 \\
\hline 42 & Wheat-Jute-Vegetable & 1320 & 0.02 & 6 & 6 \\
\hline 43 & Blackcumin-Jute-T. Aman & 1260 & 0.01 & 6 & 11 \\
\hline 44 & Vegetable-Boro-Jute & 800 & 0.01 & 2 & 3 \\
\hline 45 & Sweet Potato-Jute-T. Aman & 675 & 0.01 & 10 & 13 \\
\hline 46 & Millet (Cheena)-Jute-Fallow & 620 & 0.01 & 2 & 2 \\
\hline 47 & Potato-Boro-Jute & 590 & 0.01 & 2 & 2 \\
\hline 48 & Mu ngbean-Jute-Fallow & 300 & 0.00 & 6 & 8 \\
\hline 49 & Sesame-Jute-T. Aman & 290 & 0.00 & 2 & 2 \\
\hline 50 & Chickpea-Jute-T. Aman & 270 & 0.00 & 7 & 11 \\
\hline 51 & Mu stard-Maize-Jute & 200 & 0.00 & 1 & 1 \\
\hline 52 & Veg.-Onion-Jute-T. Aman & 200 & 0.00 & 1 & 1 \\
\hline 53 & Mungbean-Jute-T. Aman & 180 & 0.00 & 9 & 11 \\
\hline 54 & Soybean-Jute-Fallow & 140 & 0.00 & 2 & 2 \\
\hline 55 & Sunflower-Jute-Fallow & 135 & 0.00 & 3 & 3 \\
\hline 56 & Lentil-Mungbean-Jute-T. Aman & 100 & 0.00 & 1 & 1 \\
\hline 57 & Total jute & 778710 & 9.09 & - & - \\
\hline
\end{tabular}

Table 16. List of cropping patterns with tobacco and area coverage, 2014-15.

\begin{tabular}{|c|c|c|c|c|c|}
\hline & Cropping pattern & Area (ha) & $\%$ of NCA & No. of district & No. of upazila \\
\hline 01 & Tobacco-Jute-T. Aman & 15200 & 0.177 & 9 & 19 \\
\hline 02 & Tobacco-Maize-T. Aman & 7470 & 0.087 & 5 & 12 \\
\hline 03 & Tobacco-Aus-T. Aman & 6040 & 0.071 & 5 & 12 \\
\hline 04 & Tobacco-Fallow-T. Aman & 5310 & 0.062 & 7 & 15 \\
\hline 05 & Tobacco-Jute-Fallow & 4050 & 0.047 & 4 & 8 \\
\hline 06 & Tobacco-Aus-Fallow & 3180 & 0.037 & 5 & 10 \\
\hline 07 & Tobacco-Boro-T. Aman & 2300 & 0.027 & 3 & 4 \\
\hline 08 & Tobacco-Sesbania-T.Aman & 1620 & 0.019 & 1 & 2 \\
\hline 09 & Tobacco-Vegetab-Vegetab & 1500 & 0.018 & 1 & 1 \\
\hline 10 & Tobacco-Fallow-Fallow & 1045 & 0.012 & 5 & 8 \\
\hline 11 & Tobacco-Maize-Vegetable & 600 & 0.007 & 1 & 1 \\
\hline 12 & Tobacco-Sesbania & 600 & 0.007 & 1 & 1 \\
\hline 13 & Tobacco-Mung-T. Aman & 50 & 0.001 & 1 & 1 \\
\hline 14 & Tobacco-Mung-Vegetable & 40 & 0.000 & 1 & 1 \\
\hline & Total tobacco & 49005 & 0.572 & - & - \\
\hline
\end{tabular}


Vegetable is cultivated in fertile and well-drained soil often near the cities and towns. It is grown round the year in three seasons or in two seasons or in one season in a piece of land (Table 17). The most dominant vegetable containing CP is Vegetable-Vegetable-Vegetable which covered 143,270 hectares of land and $1.67 \%$ of NCA and was found to cultivate in 283 upazilas. The next CP was Vegetable-Fallow-T. Aman which covered about half of the land area of the first one and was found to cultivate in 172 upazilas. In low lying areas vegetables are usually not suitable. However, in some low lying areas vegetables are successfully cultivated in dhap or in sorjan system or in the bank of the gher. Potato, sweet potato and sweet gourd were considered separately and were not included in the vegetable containing $\mathrm{CP}^{\prime}$ s table.

Table 17. List of cropping patterns with vegetables and area coverage, 2014-15.

\begin{tabular}{|c|c|c|c|c|c|}
\hline & Cropping pattern & Area (ha) & $\%$ of NCA & No. of district & No. of upazila \\
\hline 01 & Vegetable-Vegetable-Vegetable & 143270 & 1.672 & 61 & 283 \\
\hline 02 & Vegetable-Fallow-T. Aman & 74710 & 0.872 & 45 & 170 \\
\hline 03 & Vegetable-Vegetable-Fallow & 63935 & 0.746 & 59 & 168 \\
\hline 04 & Vegetab-Vegetable-T. Aman & 51745 & 0.604 & 49 & 127 \\
\hline 05 & Vegetable-Fallow-Fallow & 31115 & 0.363 & 35 & 84 \\
\hline 06 & Vegetable-Aus-T. Aman & 28700 & 0.335 & 42 & 87 \\
\hline 07 & Vegetable-Boro-T. Aman & 21220 & 0.248 & 22 & 42 \\
\hline 08 & Vegetable-Aus-Fallow & 15940 & 0.186 & 23 & 41 \\
\hline 09 & Boro-Vegetable & 13335 & 0.156 & 27 & 36 \\
\hline 10 & Vegetable-Jute-Fallow & 13185 & 0.154 & 26 & 47 \\
\hline 11 & Potato-Vegetable-T. Aman & 12620 & 0.147 & 30 & 59 \\
\hline 12 & Onion-Vegtable-Vegetable & 11735 & 0.137 & 39 & 87 \\
\hline 13 & Vegetable-Jute-T. Aman & 11305 & 0.132 & 25 & 51 \\
\hline 14 & Chilli-Vegetable-Fallow & 8810 & 0.103 & 44 & 104 \\
\hline 15 & Boro-Vegetable-T. Aman & 8660 & 0.101 & 9 & 16 \\
\hline 16 & Fallow-Vegetable-T. Aman & 7130 & 0.083 & 16 & 29 \\
\hline 17 & Maize-Vegetable-Fallow & 7120 & 0.083 & 14 & 19 \\
\hline 18 & Vegetable-Boro-Fallow & 7100 & 0.083 & 9 & 10 \\
\hline 19 & Lentil-Vegetable-Vegetable & 6330 & 0.074 & 19 & 30 \\
\hline 20 & Wheat-Vegetable-Vegetable & 6100 & 0.071 & 16 & 21 \\
\hline 21 & Wheat-Vegetable-T. Aman & 5205 & 0.061 & 15 & 25 \\
\hline 22 & Vegetable-B. Aman & 5200 & 0.061 & 9 & 17 \\
\hline 23 & Vegetable-Maize-T. Aman & 4500 & 0.053 & 9 & 15 \\
\hline 24 & Garlic-Vegetable-Vegetable & 4385 & 0.051 & 27 & 68 \\
\hline 25 & Vegetable-Fallow-Blackgram & 3525 & 0.041 & 16 & 33 \\
\hline 26 & Vegetable-Onion-Aus & 3200 & 0.037 & 3 & 3 \\
\hline 27 & Maize-Aus-Vegetable & 3000 & 0.035 & 3 & 4 \\
\hline 28 & Lentil-Vegetable-T. Aman & 2660 & 0.031 & 11 & 11 \\
\hline 29 & Vegetable-Groundnut-T. Aman & 2660 & 0.031 & 4 & 5 \\
\hline 30 & Potato-Maize-Vegetable & 2190 & 0.026 & 4 & 4 \\
\hline 31 & Vegetable-Maize-Fallow & 2180 & 0.025 & 6 & 7 \\
\hline 32 & Coriander-Vegetable-Fallow & 2090 & 0.024 & 20 & 29 \\
\hline 33 & Wheat-Maize-Vegetable & 2000 & 0.023 & 2 & 2 \\
\hline 34 & Mustard-Jute-Vegetable & 1900 & 0.022 & 3 & 5 \\
\hline 35 & Maize-Vegetable-T. Aman & 1810 & 0.021 & 11 & 12 \\
\hline
\end{tabular}


Table 17. Continued.

\begin{tabular}{|c|c|c|c|c|c|}
\hline & Cropping pattern & Area (ha) & $\%$ of NCA & No. of district & No. of upazila \\
\hline 36 & Vegetable-Aus-Blackgram & 1610 & 0.019 & 4 & 4 \\
\hline 37 & Tobacco-Vegetable-Vegetable & 1500 & 0.018 & 1 & 1 \\
\hline 38 & Vegetable-Jute-Vegetable & 1450 & 0.017 & 5 & 5 \\
\hline 39 & Chilli-Vegetable-T. Aman & 1405 & 0.016 & 15 & 19 \\
\hline 40 & Wheat-Jute-Vegetable & 1320 & 0.015 & 6 & 6 \\
\hline 41 & Pea-Aus-Vegetable & 1310 & 0.015 & 7 & 8 \\
\hline 42 & Fieldpea-Vegetable-Fallow & 1160 & 0.014 & 4 & 5 \\
\hline 43 & Potato-Maize-Aus-Vegetable & 1030 & 0.012 & 3 & 3 \\
\hline 44 & Maize-Mungbean-Vegetable & 950 & 0.011 & 3 & 3 \\
\hline 45 & Vegetable-Boro-Aus-T. Aman & 820 & 0.010 & 4 & 5 \\
\hline 46 & Vegetable-Boro-Jute & 800 & 0.009 & 2 & 3 \\
\hline 47 & Potato-Boro-Vegetable & 700 & 0.008 & 4 & 4 \\
\hline 48 & Tobacco-Maize-Vegetable & 600 & 0.007 & 1 & 1 \\
\hline 49 & Sweet Potato-Vegetable-Fallow & 540 & 0.006 & 5 & 9 \\
\hline 50 & Potato+Maize-Vegetable-T. Aman & 210 & 0.002 & 2 & 2 \\
\hline 51 & Vegetable-Onion-Jute-T. Aman & 200 & 0.002 & 1 & 1 \\
\hline \multirow[t]{2}{*}{52} & Tobacco-Mungbean-Vegetable & 40 & 0.000 & 1 & 1 \\
\hline & Total of CP with vegetable & 606215 & 7.077 & - & - \\
\hline
\end{tabular}

If a single one $\mathrm{CP}$ is considered, its geographical distribution also produce valuable information. Table 18 presents district-wise distribution of the most dominant $\mathrm{CP}$, Boro-Fallow-T. Aman. This $\mathrm{CP}$ generally occupies medium high land area. Maximum area under this $\mathrm{CP}$ was in Mymensingh district with 188,650 hectares of land followed by Dinajpur district with 138,400 hectares of land. This $\mathrm{CP}$ was practiced in 63 districts. However, in Munshiganj, there was no Boro-Fallow-T. Aman CP. Because here, T. Aman is extremely rare. The lowest area under this $\mathrm{CP}$ was in Borguna. In Mymensingh, this CP occupied about $65.13 \%$ of its NCA as indicated by ' $\%$ of district NCA' In Mymensingh, the area under this $\mathrm{CP}$ was $8.18 \%$ of its total area of the country.

Table 18. Distribution of the most dominant Boro-Fallow- T. Aman cropping pattern and area coverage in Bangladesh, 2014-15.

\begin{tabular}{llccc}
\hline & District & Area (ha) & \% of district NCA & \% coverage of the pattern in BD \\
\hline 01 & Mymensingh & 188650 & 65.13 & 8.18 \\
02 & Dinajpur & 138400 & 49.99 & 6.00 \\
03 & Netrokona & 98300 & 49.22 & 4.26 \\
04 & Gaibandha & 96670 & 63.87 & 4.19 \\
05 & Naogaon & 96400 & 35.59 & 4.18 \\
06 & Tangail & 84000 & 36.04 & 3.64 \\
07 & Kurigram & 83500 & 55.91 & 3.62 \\
08 & Rangpur & 81300 & 46.39 & 3.53 \\
09 & Jessore & 80700 & 42.48 & 3.50 \\
10 & Bogra & 80200 & 36.10 & 3.48 \\
11 & Jamalpur & 75300 & 46.83 & 3.27 \\
12 & Sherpur & 69000 & 68.62 & 2.99 \\
13 & Nilphamari & 65300 & 53.70 & 2.83 \\
14 & Jhenaidaha & 54900 & 39.26 & 2.38 \\
15 & Chittagong & 46420 & 23.19 & 2.01 \\
16 & Thakurgaon & 45050 & 30.36 & 1.95 \\
17 & Lalmonirhat & 44600 & 45.12 & 1.93 \\
\hline
\end{tabular}


Table 18. Continued.

\begin{tabular}{|c|c|c|c|c|}
\hline & District & Area (ha) & $\%$ of district NCA & $\%$ coverage of the pattern in $\mathrm{BD}$ \\
\hline 18 & Sunamganj & 43100 & 16.12 & 1.87 \\
\hline 19 & CoxBazar & 42400 & 49.66 & 1.84 \\
\hline 20 & Satkhira & 40950 & 33.72 & 1.78 \\
\hline 21 & Kishoreganj & 40300 & 20.17 & 1.75 \\
\hline 22 & Comilla & 38710 & 18.71 & 1.68 \\
\hline 23 & Sirajganj & 38200 & 20.82 & 1.66 \\
\hline 24 & Bhola & 35650 & 19.13 & 1.55 \\
\hline 25 & Sylhet & 34200 & 16.27 & 1.48 \\
\hline 26 & B.Baria & 34010 & 24.39 & 1.47 \\
\hline 27 & Panchagarh & 32400 & 31.70 & 1.41 \\
\hline 28 & Narsingdi & 31500 & 43.60 & 1.37 \\
\hline 29 & Barisal & 31050 & 19.59 & 1.35 \\
\hline 30 & Chuadanga & 28800 & 32.56 & 1.25 \\
\hline 31 & Feni & 28800 & 40.04 & 1.25 \\
\hline 32 & Gazipur & 27100 & 31.74 & 1.18 \\
\hline 33 & Natore & 25490 & 18.85 & 1.11 \\
\hline 34 & Narail & 24950 & 33.73 & 1.08 \\
\hline 35 & Maulvibazar & 24650 & 19.34 & 1.07 \\
\hline 36 & Rajshahi & 22400 & 13.40 & 0.97 \\
\hline 37 & Magura & 20800 & 27.44 & 0.90 \\
\hline 38 & Lakshmipur & 20200 & 20.22 & 0.88 \\
\hline 39 & Khulna & 19870 & 15.64 & 0.86 \\
\hline 40 & Bagerhat & 19600 & 17.26 & 0.85 \\
\hline 41 & Joypurhat & 19200 & 24.46 & 0.83 \\
\hline 42 & Habiganj & 19070 & 11.32 & 0.83 \\
\hline 43 & Kushtia & 17900 & 15.60 & 0.78 \\
\hline 44 & Chandpur & 14690 & 15.97 & 0.64 \\
\hline 45 & Chapainawabganj & 14100 & 11.71 & 0.61 \\
\hline 46 & Noakhali & 12460 & 6.28 & 0.54 \\
\hline 47 & Meherpur & 9800 & 18.12 & 0.42 \\
\hline 48 & Khagrachhari & 9570 & 21.59 & 0.42 \\
\hline 49 & Pabna & 8650 & 4.69 & 0.38 \\
\hline 50 & Pirojpur & 6540 & 7.95 & 0.28 \\
\hline 51 & Faridpur & 6080 & 4.34 & 0.26 \\
\hline 52 & Jhalakati & 5450 & 10.50 & 0.24 \\
\hline 53 & Rajbari & 5450 & 7.17 & 0.24 \\
\hline 54 & Bandarban & 5170 & 12.69 & 0.22 \\
\hline 55 & Rangamati & 4660 & 10.67 & 0.20 \\
\hline 56 & Gopalganj & 2900 & 2.56 & 0.13 \\
\hline 57 & Narayanganj & 2200 & 5.54 & 0.10 \\
\hline 58 & Dhaka & 2040 & 2.99 & 0.09 \\
\hline 59 & Madaripur & 1800 & 2.16 & 0.08 \\
\hline 60 & Patuakhali & 1670 & 0.79 & 0.07 \\
\hline 61 & Shariatpur & 1455 & 1.87 & 0.06 \\
\hline 62 & Manikganj & 980 & 1.05 & 0.04 \\
\hline \multirow[t]{2}{*}{63} & Barguna & 350 & 0.35 & 0.02 \\
\hline & Bangladesh & 2306005 & 26.92 & 100.00 \\
\hline
\end{tabular}


Table 19 presents district-wise distribution of the second most dominant $\mathrm{CP}$, Boro-FallowFallow. This CPisusually distributed inlow lying areas like flood-prone areas, haor, bil, charland and riverbank areas. This $\mathrm{CP}$ was dominantly present in Sunamganj, Kishoreganj, Netrokona and Gopalganj districts. In Sunamganj, about $68 \%$ of its NCA was under this CP. Therefore, rest of about $32 \%$ of land remained for other $\mathrm{CPs}$ in the district. It indicated the suitability of Boro-Fallow-Fallow CP in Sunamganj as it is mainly consisted of low lying areas. This $\mathrm{CP}$ in Sunamganj occupied about $16 \%$ of the total of this CP in Bangladesh.

In Bangladesh, in some areas, there are some special conditions because of which crop cultivation is difficult in different seasons, e.g., in saline-prone areas or in tidal nonsaline areas or in extreme drought-prone areas with no irrigation facility. In these areas only T. Aman crop is cultivated in Kharif-II season which gives rise to Fallow-Fallow-T. Aman CP. Table 20 presents district-wise distribution of this $\mathrm{CP}$. The highest coverage of this $\mathrm{CP}$ was found in Chittagong district which covered 33\% of the area of this district. This CP in Chittagong district contributed $13 \%$ of this $\mathrm{CP}^{\prime}$ s total coverage of the country. In 36 districts this $\mathrm{CP}$ was noticed.

Similarly district-wise distribution of 4th, 5th and 6th dominant CPs viz, Boro-Aus- T. Aman, Fallow-Aus-T. Aman and MustardBoro-T. Aman are presented (Tables 21, 22 and 23).

Districtwise distribution of $\mathrm{AC}$ is presented in Table 24.The major ACs viz, banana, betel leaf, ginger, papaya, pineapple, sugarcane and turmeric were included in it. Individual $\mathrm{AC}$ and total AC area are mentioned in this table. AC production was the highest in Tangail which was followed by Natore and then Rangamati.

Districtwise total number of CPs, total number of crops, diversity index of $\mathrm{CPs}$ and crops are presented in Table 25. Comilla had the highest number of different CPs of 117 which was followed by Kushtia of 116 and then Jessore 101. The maximum number of 30 crops were cultivated in Munshiganj which was followed by the four districts viz, Comilla, Faridpur, Manikganj and Noakhali each of which cultivated 28 crops. Pabna had the highest CP diversity which was followed by Rajshahi and then Kushtia. Pabna had the highest crop diversity which was followed by Rajshahi and then Kushtia as well.

\section{CONCLUSION}

A better understanding of the crop growing systems is essential to formulate ecologically and socioeconomically sustainable crop production programme. Therefore, quantitative measurement of crop distribution in an area is very important. To explore the potential of $\mathrm{CPs}$ it is necessary to integrate the available technologies to increase the total yield. Extension of agricultural land is not possible in Bangladesh. Moreover, NCA is decreasing at an alarming rate because of high population pressure. Identification of major CPs and exploring their potential is important. Increase production through utilizing fallow period in $\mathrm{CP}$ can create scope for sustainable improvement of agricultural production system.

This study had made this scope of improvement by creating relevant data base. This study identified all the CPs of Bangladesh. Ecologically well adjusted CPs dominated for their area coverage in appropriate environments. Regional distribution of CPs was also identified. These results will hopefully help further planning of potentials of CPs for production.

\section{ACKNOWLEDGEMENT}

The authors acknowledge the financial support from the Mujibnagar Integrated Agricultural Development Project (BRRI Part), Ministry of Agriculture. The research team gratefully acknowledges very cordial help and cooperation from DAE personnel at all levels. They actively cooperated the research 
Table 19. Distribution of the $2^{\text {nd }}$ dominant Boro-Fallow-Fallow cropping pattern and area coverage in Bangladesh, 2014-15.

\begin{tabular}{|c|c|c|c|c|}
\hline & District & Area (ha) & $\%$ of district NCA & $\%$ coverage of the pattern in $\mathrm{BD}$ \\
\hline 01 & Sunamganj & 181600 & 67.91 & 15.94 \\
\hline 02 & Kishoreganj & 102000 & 51.04 & 8.95 \\
\hline 03 & Netrokona & 78200 & 39.16 & 6.86 \\
\hline 04 & Gopalganj & 66300 & 58.60 & 5.82 \\
\hline 05 & B.Baria & 58050 & 41.63 & 5.09 \\
\hline 06 & Habiganj & 55900 & 33.18 & 4.91 \\
\hline 07 & Sylhet & 44450 & 21.15 & 3.90 \\
\hline 08 & Noakhali & 43000 & 21.66 & 3.77 \\
\hline 09 & Comilla & 34630 & 16.74 & 3.04 \\
\hline 10 & Mymensing & 30320 & 10.47 & 2.66 \\
\hline 11 & Gazipur & 25150 & 29.46 & 2.21 \\
\hline 12 & Naogaon & 24100 & 8.90 & 2.11 \\
\hline 13 & Moulvibazar & 22850 & 17.93 & 2.01 \\
\hline 14 & Chandpur & 22100 & 24.03 & 1.94 \\
\hline 15 & Sirajganj & 21700 & 11.82 & 1.90 \\
\hline 16 & Sariatpur & 21500 & 27.56 & 1.89 \\
\hline 17 & Barisal & 21450 & 13.54 & 1.88 \\
\hline 18 & Jessore & 19030 & 10.02 & 1.67 \\
\hline 19 & Madaripur & 18700 & 22.42 & 1.64 \\
\hline 20 & Dhaka & 17750 & 26.05 & 1.56 \\
\hline 21 & Munsiganj & 16400 & 26.54 & 1.44 \\
\hline 22 & Narayanganj & 16400 & 41.33 & 1.44 \\
\hline 23 & Narsingdi & 16100 & 22.29 & 1.41 \\
\hline 24 & Bagerhat & 13700 & 12.07 & 1.20 \\
\hline 25 & Natore & 12830 & 9.49 & 1.13 \\
\hline 26 & Tangail & 12550 & 5.39 & 1.10 \\
\hline 27 & Pirojpur & 11100 & 13.49 & 0.97 \\
\hline 28 & Kurigram & 10600 & 7.10 & 0.93 \\
\hline 29 & Pabna & 9600 & 5.21 & 0.84 \\
\hline 30 & Rajshahi & 9150 & 5.48 & 0.80 \\
\hline 31 & Rangpur & 7470 & 4.26 & 0.66 \\
\hline 32 & Khulna & 7150 & 5.63 & 0.63 \\
\hline 33 & Satkhira & 7000 & 5.76 & 0.61 \\
\hline 34 & Faridpur & 6700 & 4.79 & 0.59 \\
\hline 35 & Gaibandha & 6700 & 4.43 & 0.59 \\
\hline 36 & Jhenaidah & 6560 & 4.69 & 0.58 \\
\hline 37 & Rajbari & 6550 & 8.62 & 0.57 \\
\hline 38 & Narail & 6200 & 8.38 & 0.54 \\
\hline 39 & Bogra & 6000 & 2.70 & 0.53 \\
\hline 40 & Jamalpur & 5180 & 3.22 & 0.45 \\
\hline 41 & Manikganj & 5180 & 5.55 & 0.45 \\
\hline 42 & Rangamati & 4250 & 9.73 & 0.37 \\
\hline 43 & Laxmipur & 4200 & 4.20 & 0.37 \\
\hline 44 & Chapainawabganj & 4050 & 3.36 & 0.36 \\
\hline 45 & Sherpur & 2950 & 2.93 & 0.26 \\
\hline 46 & Kushtia & 2450 & 2.13 & 0.22 \\
\hline
\end{tabular}


Table 19. Continued.

\begin{tabular}{llcrc}
\hline & District & Area (ha) & \% of district NCA & \% coverage of the pattern in BD \\
\hline 47 & Chuadanga & 2200 & 2.49 & 0.19 \\
48 & Jhalkathi & 2200 & 4.24 & 0.19 \\
49 & Lalmonirhat & 2100 & 2.12 & 0.18 \\
50 & Magura & 1970 & 2.60 & 0.17 \\
51 & Nilphamari & 1450 & 1.19 & 0.13 \\
52 & Coxsbazar & 860 & 1.01 & 0.08 \\
53 & Bandarban & 700 & 1.72 & 0.06 \\
54 & Meherpur & 600 & 1.11 & 0.05 \\
55 & Thakurgaon & 500 & 0.34 & 0.04 \\
56 & Chittagong & 400 & 0.20 & 0.04 \\
57 & Patuakhali & 350 & 0.17 & 0.03 \\
58 & Feni & 250 & 0.35 & 0.02 \\
59 & Dinajpur & 150 & 0.05 & 0.01 \\
\hline & Total & 1139530 & 13.30 & 100.00 \\
\hline
\end{tabular}

Table 20. Distribution of the $3^{\text {rd }}$ dominant Fallow-Fallow-T. Aman cropping pattern and area coverage in Bangladesh, $2014-15$.

\begin{tabular}{|c|c|c|c|c|}
\hline & District & Area (ha) & $\%$ of district NCA & $\%$ coverage of the pattern in country \\
\hline 01 & Chittagong & 66500 & 33.23 & 13.05 \\
\hline 02 & Sylhet & 58250 & 27.72 & 11.43 \\
\hline 03 & Khulna & 42000 & 33.05 & 8.24 \\
\hline 04 & Patuakhali & 35600 & 16.81 & 6.99 \\
\hline 05 & Bagerhat & 33700 & 29.68 & 6.61 \\
\hline 06 & Noakhali & 32900 & 16.57 & 6.46 \\
\hline 07 & Moulvibazar & 25070 & 19.67 & 4.92 \\
\hline 08 & Pirojpur & 24620 & 29.92 & 4.83 \\
\hline 09 & Sunamganj & 21900 & 8.19 & 4.30 \\
\hline 10 & Satkhira & 21200 & 17.46 & 4.16 \\
\hline 11 & Borguna & 19200 & 19.26 & 3.77 \\
\hline 12 & Feni & 15600 & 21.69 & 3.06 \\
\hline 13 & Habiganj & 12340 & 7.33 & 2.42 \\
\hline 14 & Chapainawab & 11800 & 9.80 & 2.32 \\
\hline 15 & Barisal & 11130 & 7.02 & 2.18 \\
\hline 16 & Coxsbazar & 10400 & 12.18 & 2.04 \\
\hline 17 & Khagrachhari & 9050 & 20.42 & 1.78 \\
\hline 18 & Jhalkathi & 8200 & 15.80 & 1.61 \\
\hline 19 & Gazipur & 7850 & 9.20 & 1.54 \\
\hline 20 & Naogaon & 7300 & 2.69 & 1.43 \\
\hline 21 & Bhola & 7200 & 3.86 & 1.41 \\
\hline 22 & Bandarban & 5030 & 12.35 & 0.99 \\
\hline 23 & Netrokona & 5000 & 2.50 & 0.98 \\
\hline 24 & Rangamati & 3530 & 8.08 & 0.69 \\
\hline 25 & Comilla & 3430 & 1.66 & 0.67 \\
\hline 26 & Mymensing & 2600 & 0.90 & 0.51 \\
\hline 27 & Kishoreganj & 2260 & 1.13 & 0.44 \\
\hline 28 & Tangail & 1700 & 0.73 & 0.33 \\
\hline 29 & Bogra & 1550 & 0.70 & 0.30 \\
\hline 30 & Sherpur & 1320 & 1.31 & 0.26 \\
\hline 31 & Rangpur & 350 & 0.20 & 0.07 \\
\hline
\end{tabular}


Table 20. Continued.

\begin{tabular}{llrrr}
\hline & District & Area (ha) & \% of district NCA & \% coverage of the pattern in country \\
\hline 32 & Jessore & 300 & 0.16 & 0.06 \\
33 & Dinajpur & 200 & 0.07 & 0.04 \\
34 & Laxmipur & 200 & 0.20 & 0.04 \\
35 & Narsingdi & 100 & 0.14 & 0.02 \\
36 & Panchgarh & 100 & 0.10 & 0.02 \\
\hline & & Total & 509480 & 5.95 \\
\hline
\end{tabular}

Table 21. Distribution of the $4^{\text {th }}$ dominant Boro-Aus-T. Aman cropping pattern and area coverage in Bangladesh, 2014-15.

\begin{tabular}{|c|c|c|c|c|}
\hline & District & Area (ha) & $\%$ of district NCA & $\%$ coverage of the pattern in country \\
\hline 01 & Comilla & 50680 & 24.49 & 24.25 \\
\hline 02 & Bogra & 14290 & 6.43 & 6.84 \\
\hline 03 & Habiganj & 14200 & 8.43 & 6.79 \\
\hline 04 & Naogaon & 13600 & 5.02 & 6.51 \\
\hline 05 & Kishoreganj & 12350 & 6.18 & 5.91 \\
\hline 06 & Jessore & 11500 & 6.05 & 5.50 \\
\hline 07 & Bhola & 10000 & 5.37 & 4.78 \\
\hline 08 & Chandpur & 9280 & 10.09 & 4.44 \\
\hline 09 & Chittagong & 9250 & 4.62 & 4.43 \\
\hline 10 & Mymensing & 8000 & 2.76 & 3.83 \\
\hline 11 & Kushtia & 6650 & 5.79 & 3.18 \\
\hline 12 & Chapainawabganj & 6640 & 5.52 & 3.18 \\
\hline 13 & Brahmanbaria & 4870 & 3.49 & 2.33 \\
\hline 14 & Rangpur & 4570 & 2.61 & 2.19 \\
\hline 15 & Coxsbazar & 3500 & 4.10 & 1.67 \\
\hline 16 & Natore & 3400 & 2.51 & 1.63 \\
\hline 17 & Moulvibazar & 3040 & 2.38 & 1.45 \\
\hline 18 & Pabna & 2950 & 1.60 & 1.41 \\
\hline 19 & Satkhira & 2780 & 2.29 & 1.33 \\
\hline 20 & Sirajganj & 2450 & 1.34 & 1.17 \\
\hline 21 & Laxmipur & 2200 & 2.20 & 1.05 \\
\hline 22 & Jhenaidah & 1700 & 1.22 & 0.81 \\
\hline 23 & Rajshahi & 1650 & 0.99 & 0.79 \\
\hline 24 & Dinajpur & 1575 & 0.57 & 0.75 \\
\hline 25 & Sherpur & 1350 & 1.34 & 0.65 \\
\hline 26 & Gazipur & 1120 & 1.31 & 0.54 \\
\hline 27 & Noakhali & 1070 & 0.54 & 0.51 \\
\hline 28 & Bagerhat & 950 & 0.84 & 0.45 \\
\hline 29 & Kurigram & 880 & 0.59 & 0.42 \\
\hline 30 & Sylhet & 600 & 0.29 & 0.29 \\
\hline 31 & Magura & 500 & 0.66 & 0.24 \\
\hline 32 & Khagrachhari & 200 & 0.45 & 0.10 \\
\hline 33 & Rangamati & 200 & 0.46 & 0.10 \\
\hline 34 & Gaibandha & 170 & 0.11 & 0.08 \\
\hline 35 & Khulna & 165 & 0.13 & 0.08 \\
\hline 36 & Jhalkathi & 100 & 0.19 & 0.05 \\
\hline 37 & Pirojpur & 100 & 0.12 & 0.05 \\
\hline
\end{tabular}


Table 21. Continued.

\begin{tabular}{llccc}
\hline & District & Area (ha) & $\%$ of district NCA & \% coverage of the pattern in country \\
\hline 38 & Sariatpur & 100 & 0.13 & 0.05 \\
39 & Narsingdi & 90 & 0.12 & 0.04 \\
40 & Bandarban & 80 & 0.20 & 0.04 \\
41 & Feni & 50 & 0.07 & 0.02 \\
42 & Jamalpur & 50 & 0.03 & 0.02 \\
43 & Rajbari & 50 & 0.07 & 0.02 \\
44 & Patuakhali & 30 & 0.01 & 0.01 \\
45 & Nilphamari & 20 & 0.02 & 0.01 \\
46 & Joypurhat & 10 & 0.01 & 0.00 \\
47 & Tangail & 5 & 0.00 & 0.00 \\
\hline
\end{tabular}

Table 22. Distribution of the $5^{\text {th }}$ dominant Fallow-Aus-T. Aman cropping pattern and area coverage in Bangladesh, 2014-15.

\begin{tabular}{|c|c|c|c|c|}
\hline & District & Area (ha) & $\%$ of district NCA & $\%$ coverage of the pattern in country \\
\hline 01 & Sylhet & 35600 & 16.94 & 18.42 \\
\hline 02 & Moulvibazar & 33900 & 26.59 & 17.54 \\
\hline 03 & Habiganj & 23825 & 14.14 & 12.33 \\
\hline 04 & Jhalkathi & 12300 & 23.70 & 6.36 \\
\hline 05 & Noakhali & 12200 & 6.15 & 6.31 \\
\hline 06 & Chittagong & 9290 & 4.64 & 4.81 \\
\hline 07 & Pirojpur & 8800 & 10.70 & 4.55 \\
\hline 08 & Borguna & 8540 & 8.57 & 4.42 \\
\hline 09 & Barisal & 8400 & 5.30 & 4.35 \\
\hline 10 & Mymensing & 8400 & 2.90 & 4.35 \\
\hline 11 & Rajshahi & 4500 & 2.69 & 2.33 \\
\hline 12 & Feni & 4200 & 5.84 & 2.17 \\
\hline 13 & Sunamganj & 3730 & 1.39 & 1.93 \\
\hline 14 & Comilla & 3350 & 1.62 & 1.73 \\
\hline 15 & Bhola & 2900 & 1.56 & 1.50 \\
\hline 16 & Kishoreganj & 2200 & 1.10 & 1.14 \\
\hline 17 & Patuakhali & 2060 & 0.97 & 1.07 \\
\hline 18 & Bagerhat & 1740 & 1.53 & 0.90 \\
\hline 19 & Naogaon & 1620 & 0.60 & 0.84 \\
\hline 20 & Laxmipur & 1200 & 1.20 & 0.62 \\
\hline 21 & Khagrachhari & 1110 & 2.50 & 0.57 \\
\hline 22 & Sherpur & 1100 & 1.09 & 0.57 \\
\hline 23 & Brahmanbaria & 810 & 0.58 & 0.42 \\
\hline 24 & Netrokona & 410 & 0.21 & 0.21 \\
\hline 25 & Satkhira & 380 & 0.31 & 0.20 \\
\hline 26 & Coxsbazar & 250 & 0.29 & 0.13 \\
\hline 27 & Rangamati & 210 & 0.48 & 0.11 \\
\hline 28 & Khulna & 100 & 0.08 & 0.05 \\
\hline 29 & Tangail & 100 & 0.04 & 0.05 \\
\hline \multirow[t]{2}{*}{30} & Gazipur & 50 & 0.06 & 0.03 \\
\hline & Total & 193275 & 2.26 & 100.00 \\
\hline
\end{tabular}


Table 23. Distribution of the $6^{\text {th }}$ dominant Mustard-Boro-T. Aman cropping pattern and area coverage in Bangladesh, 2014-15.

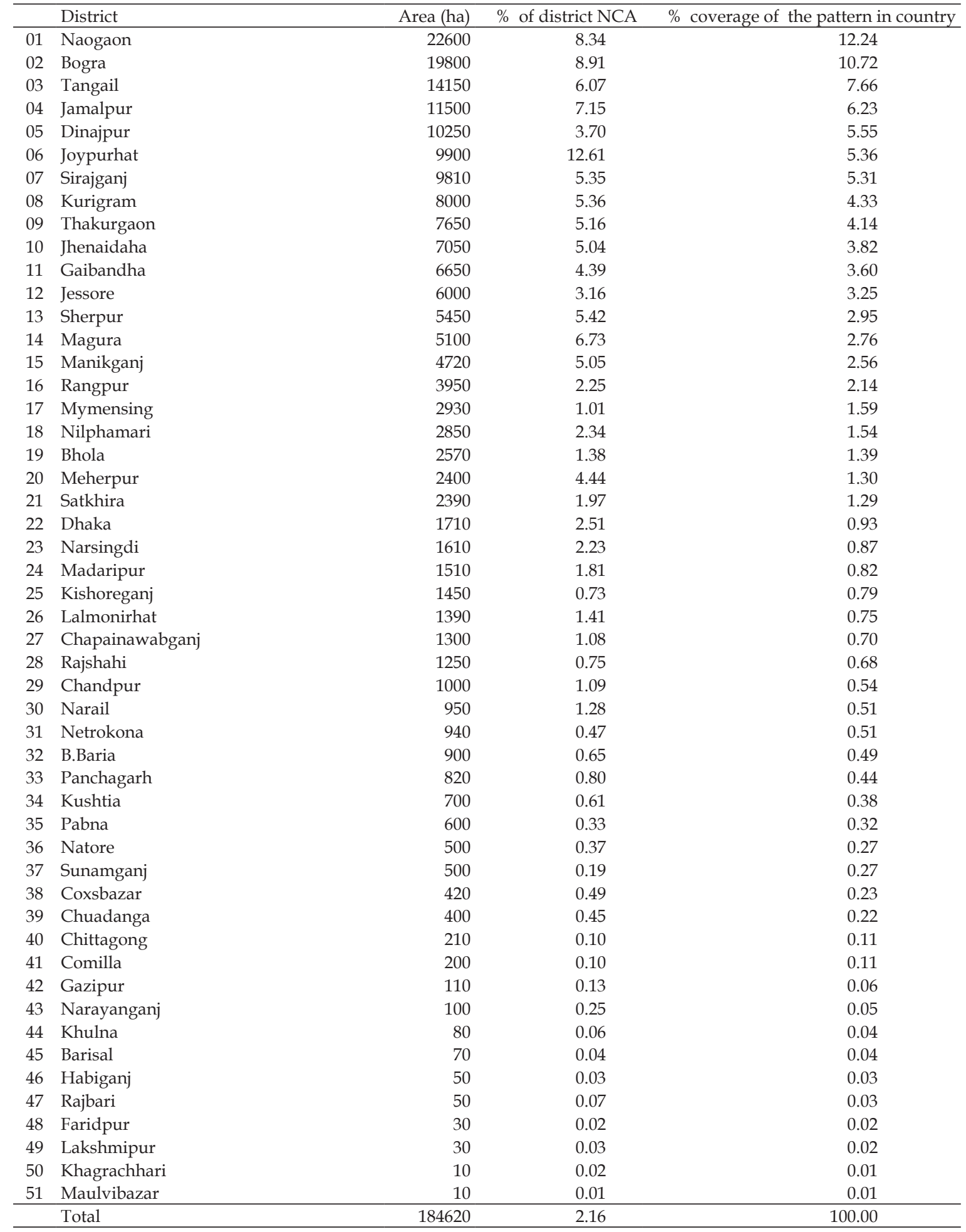


Table 24. Distribution of annual crops in different districts of Bangladesh, 2014-15.

\begin{tabular}{|c|c|c|c|c|c|c|c|c|c|}
\hline & District & Banana & Betel leaf & Ginger & Papaya & Pineapple & Sugarcane & Turmeric & Total \\
\hline 01 & Brahmanbaria & 17 & 0 & 75 & 34 & 0 & 80 & 84 & 290 \\
\hline 02 & Bagerhat & 1290 & 1080 & 0 & 110 & 0 & 920 & 30 & 3430 \\
\hline 03 & Bandarban & 7120 & 0 & 1410 & 840 & 2835 & 215 & 1260 & 13680 \\
\hline 04 & Barguna & 150 & 355 & 0 & 65 & 3 & 57 & 0 & 630 \\
\hline 05 & Barisal & 1235 & 2678 & 6 & 465 & 0 & 600 & 111 & 5095 \\
\hline 06 & Bhola & 2735 & 510 & 60 & 490 & 15 & 1060 & 335 & 5205 \\
\hline 07 & Bogra & 973 & 0 & 128 & 165 & 0 & 84 & 170 & 1520 \\
\hline 08 & Chandpur & 195 & 200 & 63 & 15 & 0 & 410 & 62 & 945 \\
\hline 09 & Chapainawabganj & 590 & 0 & 100 & 220 & 0 & 10150 & 100 & 11160 \\
\hline 10 & Chittagong & 2320 & 420 & 710 & 842 & 162 & 381 & 345 & 5180 \\
\hline 11 & Chuadanga & 480 & 2000 & 10 & 10 & 0 & 645 & 255 & 3400 \\
\hline 12 & Comilla & 286 & 130 & 37 & 71 & 30 & 360 & 66 & 980 \\
\hline 13 & Coxsbazar & 464 & 2705 & 80 & 136 & 25 & 350 & 70 & 3830 \\
\hline 14 & Dhaka & 310 & 0 & 30 & 270 & 0 & 60 & 70 & 740 \\
\hline 15 & Dinajpur & 1095 & 40 & 280 & 55 & 0 & 2050 & 625 & 4145 \\
\hline 16 & Faridpur & 1315 & 20 & 0 & 480 & 0 & 3525 & 250 & 5590 \\
\hline 17 & Feni & 95 & 0 & 0 & 35 & 0 & 150 & 0 & 280 \\
\hline 18 & Gaibandha & 998 & 130 & 190 & 47 & 0 & 2415 & 330 & 4110 \\
\hline 19 & Gazipur & 3575 & 0 & 305 & 2205 & 120 & 775 & 540 & 7520 \\
\hline 20 & Gopalganj & 515 & 5 & 0 & 240 & 0 & 1020 & 10 & 1790 \\
\hline 21 & Habiganj & 157 & 120 & 132 & 98 & 200 & 310 & 183 & 1200 \\
\hline 22 & Jamalpur & 550 & 40 & 70 & 0 & 120 & 1935 & 95 & 2810 \\
\hline 23 & Jessore & 1050 & 1130 & 50 & 135 & 0 & 475 & 1180 & 4020 \\
\hline 24 & Jhalakati & 1515 & 580 & 3 & 420 & 0 & 190 & 122 & 2830 \\
\hline 25 & Jhenaidaha & 4335 & 2335 & 5 & 495 & 0 & 1880 & 1100 & 10150 \\
\hline 26 & Joypurhat & 562 & 0 & 59 & 31 & 0 & 520 & 328 & 1500 \\
\hline 27 & Khagrachhari & 1800 & 0 & 3020 & 160 & 415 & 345 & 4480 & 10220 \\
\hline 28 & Khulna & 190 & 570 & 10 & 45 & 0 & 80 & 185 & 1080 \\
\hline 29 & Kishoreganj & 747 & 0 & 77 & 232 & 0 & 267 & 167 & 1490 \\
\hline 30 & Kurigram & 713 & 5 & 350 & 72 & 0 & 270 & 350 & 1760 \\
\hline 31 & Kushtia & 2380 & 2120 & 25 & 80 & 0 & 1925 & 750 & 7280 \\
\hline 32 & Laksmipur & 160 & 20 & 0 & 40 & 0 & 140 & 40 & 400 \\
\hline 33 & Lalmonirhat & 160 & 0 & 330 & 10 & 0 & 110 & 680 & 1290 \\
\hline 34 & Madaripur & 210 & 192 & 0 & 140 & 0 & 493 & 5 & 1040 \\
\hline 35 & Magura & 455 & 154 & 0 & 71 & 0 & 200 & 780 & 1660 \\
\hline 36 & Manikganj & 110 & 0 & 40 & 50 & 0 & 2100 & 50 & 2350 \\
\hline 37 & Maulvibazar & 480 & 25 & 255 & 20 & 750 & 20 & 270 & 1820 \\
\hline 38 & Meherpur & 1460 & 0 & 0 & 0 & 0 & 170 & 710 & 2340 \\
\hline 39 & Munsiganj & 55 & 0 & 15 & 10 & 0 & 85 & 25 & 190 \\
\hline 40 & Mymensingh & 435 & 267 & 450 & 136 & 228 & 2669 & 1875 & 6060 \\
\hline 41 & Naogaon & 730 & 0 & 125 & 150 & 0 & 1225 & 270 & 2500 \\
\hline 42 & Narail & 255 & 235 & 5 & 35 & 0 & 825 & 315 & 1670 \\
\hline 43 & Narayanganj & 40 & 0 & 0 & 30 & 0 & 110 & 0 & 180 \\
\hline 44 & Narsingdi & 2140 & 300 & 0 & 160 & 190 & 60 & 0 & 2850 \\
\hline 45 & Natore & 1110 & 10 & 50 & 110 & 0 & 15040 & 920 & 17240 \\
\hline 46 & Netrokona & 27 & 0 & 82 & 32 & 0 & 27 & 142 & 310 \\
\hline 47 & Nilphamari & 210 & 0 & 870 & 45 & 0 & 175 & 1010 & 2310 \\
\hline
\end{tabular}


Table 24. Continued.

\begin{tabular}{|c|c|c|c|c|c|c|c|c|c|}
\hline & District & Banana & Betel leaf & Ginger & Papaya & Pineapple & Sugarcane & Turmeric & Total \\
\hline 48 & Noakhali & 1350 & 10 & 0 & 68 & 0 & 112 & 0 & 1540 \\
\hline 49 & Pabna & 305 & 10 & 0 & 90 & 0 & 4605 & 910 & 5920 \\
\hline 50 & Panchagarh & 175 & 20 & 260 & 5 & 0 & 1150 & 700 & 2310 \\
\hline 51 & Patuakhali & 353 & 634 & 0 & 90 & 0 & 157 & 111 & 1345 \\
\hline 52 & Pirojpur & 880 & 478 & 8 & 105 & 5 & 373 & 281 & 2130 \\
\hline 53 & Rajbari & 600 & 0 & 0 & 25 & 0 & 1245 & 490 & 2360 \\
\hline 54 & Rajshahi & 1080 & 295 & 0 & 590 & 0 & 8045 & 410 & 10420 \\
\hline 55 & Rangamati & 7666 & 0 & 2420 & 305 & 1245 & 199 & 2545 & 14380 \\
\hline 56 & Rangpur & 1285 & 50 & 865 & 50 & 0 & 1200 & 900 & 4350 \\
\hline 57 & Satkhira & 460 & 445 & 10 & 59 & 21 & 132 & 703 & 1830 \\
\hline 58 & Shariatpur & 365 & 80 & 0 & 120 & 0 & 635 & 60 & 1260 \\
\hline 59 & Sherpur & 40 & 5 & 270 & 0 & 0 & 25 & 420 & 760 \\
\hline 60 & Sirajganj & 280 & 0 & 200 & 120 & 0 & 1670 & 170 & 2440 \\
\hline 61 & Sunamganj & 145 & 0 & 28 & 100 & 10 & 195 & 32 & 510 \\
\hline 62 & Sylhet & 68 & 0 & 5 & 759 & 203 & 50 & 15 & 1100 \\
\hline 63 & Tangail & 6085 & 30 & 1685 & 275 & 7530 & 955 & 3690 & 20250 \\
\hline \multirow[t]{2}{*}{64} & Thakurgaon & 290 & 0 & 100 & 50 & 0 & 1950 & 400 & 2790 \\
\hline & Bangladesh & 69221 & 20433 & 15358 & 12413 & 14107 & 79651 & 32582 & 243765 \\
\hline
\end{tabular}

Table 25. Crop diversity and cropping intensity of different districts in Bangladesh, 2014-15.

\begin{tabular}{llccccc}
\hline & District & $\begin{array}{c}\text { No. of identified } \\
\text { pattern }\end{array}$ & No. of crop & $\begin{array}{c}\text { Diversity index for } \\
\text { cropping pattern }\end{array}$ & $\begin{array}{c}\text { Crop diversity } \\
\text { index (CDI) }\end{array}$ & C.I. (\%) \\
\hline 01 & B.Baria & 62 & 18 & 0.757 & 0.883 & 165 \\
02 & Bagerhat & 53 & 20 & 0.848 & 0.921 & 161 \\
03 & Bandarban & 49 & 24 & 0.953 & 0.963 & 138 \\
04 & Barguna & 29 & 17 & 0.868 & 0.939 & 218 \\
05 & Barisal & 74 & 20 & 0.911 & 0.955 & 192 \\
06 & Bhola & 61 & 25 & 0.933 & 0.972 & 228 \\
07 & Bogra & 96 & 27 & 0.803 & 0.925 & 248 \\
08 & Chandpur & 22 & 0.880 & 0.945 & 190 \\
09 & ChapaiN.ganj & 74 & 18 & 0.944 & 0.975 & 217 \\
10 & Chittagong & 62 & 25 & 0.826 & 0.924 & 180 \\
11 & Chuadanga & 63 & 23 & 0.867 & 0.945 & 224 \\
12 & Comilla & 48 & 28 & 0.868 & 0.932 & 210 \\
13 & CoxBazar & 117 & 22 & 0.725 & 0.856 & 197 \\
14 & Dhaka & 44 & 25 & 0.889 & 0.937 & 196 \\
15 & Dinajpur & 67 & 22 & 0.723 & 0.889 & 228 \\
16 & Faridpur & 75 & 28 & 0.942 & 0.972 & 225 \\
17 & Feni & 96 & 22 & 0.777 & 0.879 & 183 \\
18 & Gaibandha & 34 & 23 & 0.583 & 0.807 & 210 \\
19 & Gazipur & 52 & 26 & 0.801 & 0.880 & 161 \\
20 & Gopalganj & 47 & 0.643 & 0.824 & 146 \\
\hline
\end{tabular}


Table 25. Continued.

\begin{tabular}{|c|c|c|c|c|c|c|}
\hline & District & $\begin{array}{l}\text { No. of identified } \\
\text { pattern }\end{array}$ & No. of crop & $\begin{array}{l}\text { Diversity index for } \\
\text { cropping pattern }\end{array}$ & $\begin{array}{l}\text { Crop diversity } \\
\text { index (CDI) }\end{array}$ & C.I. (\%) \\
\hline 21 & Habiganj & 46 & 21 & 0.827 & 0.916 & 170 \\
\hline 22 & Jamalpur & 77 & 22 & 0.762 & 0.898 & 220 \\
\hline 23 & Jessore & 101 & 25 & 0.799 & 0.920 & 225 \\
\hline 24 & Jhalakati & 40 & 21 & 0.891 & 0.944 & 189 \\
\hline 25 & Jhenaidaha & 76 & 26 & 0.833 & 0.931 & 223 \\
\hline 26 & Joypurhat & 45 & 19 & 0.697 & 0.879 & 267 \\
\hline 27 & Khagrachhari & 47 & 23 & 0.900 & 0.929 & 151 \\
\hline 28 & Khulna & 52 & 21 & 0.839 & 0.922 & 159 \\
\hline 29 & Kishoreganj & 91 & 21 & 0.694 & 0.852 & 156 \\
\hline 30 & Kurigram & 78 & 25 & 0.673 & 0.854 & 214 \\
\hline 31 & Kushtia & 116 & 26 & 0.955 & 0.982 & 240 \\
\hline 32 & Lakshmipur & 44 & 21 & 0.878 & 0.945 & 240 \\
\hline 33 & Lalmonirhat & 44 & 22 & 0.750 & 0.897 & 223 \\
\hline 34 & Madaripur & 57 & 24 & 0.910 & 0.967 & 204 \\
\hline 35 & Magura & 55 & 26 & 0.878 & 0.980 & 251 \\
\hline 36 & Manikganj & 75 & 28 & 0.917 & 0.958 & 212 \\
\hline 37 & Maulvibazar & 47 & 20 & 0.819 & 0.894 & 168 \\
\hline 38 & Meherpur & 40 & 21 & 0.932 & 0.973 & 239 \\
\hline 39 & Munsiganj & 38 & 30 & 0.835 & 0.915 & 177 \\
\hline 40 & Mymensingh & 71 & 24 & 0.562 & 0.779 & 199 \\
\hline 41 & Naogaon & 90 & 23 & 0.848 & 0.936 & 221 \\
\hline 42 & Narail & 37 & 21 & 0.850 & 0.932 & 210 \\
\hline 43 & Narayanganj & 45 & 27 & 0.800 & 0.906 & 158 \\
\hline 44 & Narsingdi & 54 & 23 & 0.753 & 0.861 & 181 \\
\hline 45 & Natore & 68 & 22 & 0.939 & 0.972 & 210 \\
\hline 46 & Netrokona & 54 & 20 & 0.603 & 0.746 & 160 \\
\hline 47 & Nilphamari & 59 & 21 & 0.695 & 0.875 & 224 \\
\hline 48 & Noakhali & 49 & 28 & 0.892 & 0.953 & 178 \\
\hline 49 & Pabna & 90 & 29 & 0.970 & 0.987 & 230 \\
\hline 50 & Panchagarh & 65 & 24 & 0.862 & 0.938 & 216 \\
\hline 51 & Patuakhali & 51 & 22 & 0.893 & 0.951 & 203 \\
\hline 52 & Pirojpur & 50 & 24 & 0.858 & 0.930 & 162 \\
\hline 53 & Rajbari & 50 & 26 & 0.937 & 0.971 & 234 \\
\hline 54 & Rajshahi & 99 & 27 & 0.964 & 0.984 & 221 \\
\hline 55 & Rangamati & 41 & 25 & 0.944 & 0.954 & 128 \\
\hline 56 & Rangpur & 70 & 22 & 0.768 & 0.907 & 227 \\
\hline 57 & Satkhira & 62 & 22 & 0.840 & 0.923 & 193 \\
\hline 58 & Shariatpur & 72 & 25 & 0.905 & 0.963 & 178 \\
\hline 59 & Sherpur & 44 & 20 & 0.522 & 0.785 & 213 \\
\hline 60 & Sirajganj & 100 & 30 & 0.891 & 0.950 & 206 \\
\hline 61 & Sunamganj & 52 & 24 & 0.506 & 0.656 & 123 \\
\hline 62 & Sylhet & 38 & 21 & 0.820 & 0.894 & 151 \\
\hline 63 & Tangail & 89 & 29 & 0.842 & 0.922 & 204 \\
\hline \multirow[t]{2}{*}{64} & Thakurgaon & 50 & 23 & 0.887 & 0.955 & 240 \\
\hline & Bangladesh & 316 & 48 & 0.896 & 0.952 & 200 \\
\hline
\end{tabular}


team and sincerely participated in every stage of the study especially in all the workshops conducted separately in 64 districts during the study. They provided us with the information based on which the study was conducted. The research team is very thankful to the former DG of BRRI, Dr Jiban Krishna Biswas and also the former DG of DAE, Mr Mamtajul Karim for their advice and support to the team.

\section{REFERENCES}

Ali, M Y. 2014. Farming Systems of Bangladesh: Poverty Escape Pathways and Livelihood Improvement. Pub. by: Anjuman Ara, D-186/7, Nazrul Sarani, Middle Sayabithi, Joorpukur, Gazipur 1700, Bangladesh.

Anonymous. 2016a. Statistical Year Book of Bangladesh 2015. Bangladesh Bureau of Statistics, Statistics and Informatics Division, Ministry of Planning.

Anonymous. 2016b. Year Book of Agricultural Statistics-2015. Bangladesh Bureau of Statistics, Statistics and Informatics Division, Ministry of Planning.

Catling, H D, D W Puckridge and D H Lambers. 1988. The environment of Asian deepwater rice. In: Proceeding of the 1987 international deepwater rice workshop. International Rice Research Institute, P. O. Box 933, Manila, Philippines.

Chowdhury, M K A, M R I Mondal and M A Quayyum. 2008. Proceedings of the National Workshop on Research and Development of Multiple Cropping Systems in Bangladesh held at Bangladesh Agriculture Research Council, Farmgate, Dhaka-1215, Bangladesh, held on 23-24 April, 2008.

FAO, 1988. Land Resources Appraisal of Bangladesh for Agricultural Development- Report 2: Agroecological regions of Bangladesh. Food and Agriculture Organization of the United Nations, Rome, Italy, 570p

Hasan, M N, M S Hossain, M A Bari and M R Islam. 2013. Agricultural land availability in Bangladesh. SRDI, Dhaka, Bangladesh.

Hossain, M I, M Y Rahman and P Bala. 2016. Agricultural adaptations for climate change in Dacope upazila of
Bangladesh. Bangladesh Res. Pub. J. 12 (1): 60-70.

Kabir, MS, M U Salam, A Chowdhury, N M F Rahman, K M Iftekharuddaula, MS Rahman, M H Rashid, SS Dipti, A Islam, M A Latif, A K M S Islam, M M Hossain, B Nessa, T H Ansari, M A Ali and J K Biswas. 2015. Rice vision for Bangladesh: 2050 and beyond. Bangladesh Rice J. 19(2): 1-18.

Kshirsagar, KG, S Pandey and MR Bellon. 1997. Farmers' perception, varietal characteristics and technology adoption: the case of rainfed village in eastern India. Discussion paper5/97. Social Sciences Division, IRRI, Philippines.

Khush G S. 1984. Terminology for rice growing environments. In: Terminology for rice growing environments. International Rice Research Institute, P. O. Box 933, Manila, Philippines.

Miah, N M, A U Ahmed and B A A Mustafi. 2004. Adopting modern rice technologies in flood-prone areas: status, constraints and opportunities. In: Rice research and development in the flood-prone ecosystem. Proceedings of the international workshop on floodprone rice systems held in Gazipur, Bangladesh, 9-11 January 2001. International Rice Research Institute, P. O. Box 933, Manila, Philippines.

Nur-E-Elahi, A H Khan, M R Siddique, A Saha, M Nasim, M I U Mollah and S M Shahidullah. 2001. Existing Cropping Patterns of Bangladesh, Potential Technologies and Strategies for Improving Systems Productivity. In: Proceedings of the Workshop on Modern Rice Cultivation in Bangladesh, held on 14-16 February 1999 at Bangladesh Rice Research Institute, Gazipur. BRRI Publication No. 132.

Rahman, M M, M A Aziz, M A Musa and J Kumar. 1995. Prospects of pulse crops in the rice-based cropping system in Bangladesh. In: Fragile lives in fragile ecosystems. Proceedings of the international rice research conference, 13-17 February 1995. International Rice Research Institute, P.O. Box 933, Manila, Philippines.

Shahidullah, S M, M S A Talukder, M S Kabir, A H Khan and N E Elahi. 2006. Cropping patterns in the South East Coastal Region of Bangladesh. J. Agric. Rural Dev. 4(1\&2): 53-60.

Sujatha, P J, S Punithavathi, Tamilenthi and R Baskaran. 2011. Land use pattern and cropping pattern of Orthanadu Block, Thanjavur district, Tamil Nadu using GIS. J. Experimental Sci. 2(5): 19-23. 
